\title{
Implementation of Multi-Layer Active Structural-Acoustic Transmission Control
}

by

\author{
Jonathan K. Wong
}

Submitted to the Department of Aeronautics and Astronautics in partial fulfillment of the requirements for the degree of

Master of Science in Aeronautics and Astronautics

at the

MASSACHUSETTS INSTITUTE OF TECHNOLOGY

June 2001

(C)Massachusetts Institute of Technology, MMI. All rights reserved.

Author........................

Department of Aeronautics and Astoronautics

May 25, 2001

Certified by

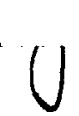

John-Pál B. Clarke Assistant Professor of Aeronautics and Astronautics

Thesis Supervisor

Accepted by ............

Wallace E. Vander Velde

Professor of Aeronautics and Astronautics Chairman, Department Graduate Committee

MASSACHUSETTS INSTITUTE OF TECHNOLOGY

SEP 112001

LIBRARIES 


\title{
Implementation of Multi-Layer Active Structural-Acoustic Transmission Control
}

\author{
by \\ Jonathan K. Wong \\ Submitted to the Department of Aeronautics and Astronautics \\ on May 25, 2001, in partial fulfillment of the \\ requirements for the degree of \\ Master of Science in Aeronautics and Astronautics
}

\begin{abstract}
Structural-acoustic vibration is one of the major sources of payload failure during launch. Past research on the topic of structural-acoustic vibration control has focused on actuating a single structural layer to minimize the amount of energy flowing across the boundary. This thesis investigates the use of a dual layer approach to address the structural-acoustic problem and compares the results to a single layer approach.

Four different controller configurations were used to experimentally determine which configuration is best suited for multi-layer transmission control. The four controller configurations were the Multi-Input Multi-Output (MIMO) Controller, the Successive Loop Closure (SLC) Controller, the Interference Controller and the Power Diode Controller. The MIMO Controller used a single state-space controller to actively control the two active plates, while the other three configurations used a single controller to actively control each plate. The SLC Controller and the Interference Controller are different because the design order of the two plate controllers for the Interference Controller is reversed compared to the SLC Controller. The Power Diode Controller implements a power diode on one of the two structural plates. The power diode is designed to attenuate acoustic transmission across the plate in one direction only as compared to typical controllers that attenuate acoustic transmission across the plate in both directions.

Although the four controller configurations were different, they shared a common goal-to minimize the system's response over the $40-1000 \mathrm{~Hz}$ broadband frequency region. The SLC Controller performed the best by posting a $2.08 \mathrm{~dB}$ reduction across the broadband region, compared to a $1.06 \mathrm{~dB}$ reduction posted by the worst performing controller. These reductions only refer to the differences in open and closed loop performances of the dual layer configuration. Compared to a passive single layer system with the same thickness as the two separate layers combined, the SLC Controller posted a $3.02 \mathrm{~dB}$ reduction for the broadband metric.
\end{abstract}

Thesis Supervisor: John-Paul B. Clarke

Title: Assistant Professor of Aeronautics and Astronautics 


\section{Acknowledgments}

This work would not have been possible without the help and support of many people. First, I would like to thank Professor J. P. Clarke, who provided guidance and advice that proved invaluable in defining the scope of the research and in providing many suggestions whenever the direction of further research was unclear. I thank my project partner, Chris Merchant, for helping me setup and troubleshoot the experimental test chamber as well as being a sounding-board for solutions and ideas. I also thank my family and my fiancée, Barbara, for providing unconditional love and support for the last two years. Lastly, I would like to acknowledge the AFOSR, whose grant made this research financially feasible. 


\section{Contents}

1 Introduction $\quad 13$

1.1 Motivation . . . . . . . . . . . . . . . 13

1.2 Background ............................ 14

1.2.1 Active Control Techniques . . . . . . . . . . 15

1.2.2 Passive Control Techniques . . . . . . . . . . 16

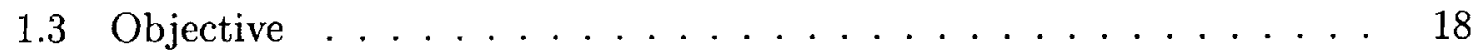

1.4 Previous Work . . . . . . . . . . . . . . 18

1.5 Thesis Outline . . . . . . . . . . . . . . 20

2 Experimental Setup 23

2.1 Test Chamber . . . . . . . . . . . . . . . . 23

2.1 .1 Dimensions ...................... 23

2.1 .2 Excitation Sources . . . . . . . . . . . . 24

2.1.3 Test Chamber Configurations ........... . 24

2.1.4 Structural Plates ............... 26

2.2 Electronics............................ 27

2.2 .1 Amplifiers .................. 27

2.2 .2 Measurement Devices . . . . . . . . . . . . 28

2.2.3 Function Generator . . . . . . . . . . . . 29

2.2.4 Real Time Computer . . . . . . . . . . . . 30

3 Structural-Acoustic Modeling

3.1 Measurement Model ................... 31 
3.1.1 Transfer Function Matrix . . . . . . . . . . . . . 32

3.1 .2 State-Space Model ... . . . . . . . . . . . 32

4 Linear Quadratic Gaussian Controller 35

4.1 LQG Controller Formulation . . . . . . . . . . . . . . . . 36

4.2 LQR and Kalman Filter Interconnection . . . . . . . . . . . . . . . . 38

5 Experimental Results $\quad 39$

5.1 Passive Configuration Performance . . . . . . . . . . . . . . 39

5.2 Active Control . . . . . . . . . . . . . . . . 42

5.2.1 MIMO Controller .................. . . 42

5.2 .2 Successive Loop Closure Controller . . . . . . . . . . . . 51

5.2 .3 Interference Controller ................ 62

5.2.4 Power Diode Controller . . . . . . . . . . . . . . . . 70

5.2 .5 Single Layer Reference . . . . . . . . . . . . . . 85

5.2 .6 Inter-plate Spacing . . . . . . . . . . . . . . . 85

6 Conclusions $\quad 87$

6.1 Summary . . . . . . . . . . . . . . . . . 87

6.2 Recommendations for Further Work . . . . . . . . . . . . . . . . 89 


\section{List of Figures}

2-1 Test Chamber in Single Layer Configuration . . . . . . . . . . 24

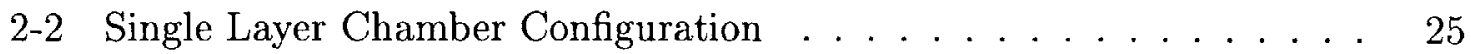

2-3 Dual Layer Chamber Configuration . . . . . . . . . . . . 26

2-4 Diode Chamber Configuration . . . . . . . . . . . . . 27

$2-5$ Structural Plate with PZT Patch . . . . . . . . . . 28

2-6 Block Diagram of the Experimental Setup . . . . . . . . . . . 29

3-1 The Four Block Problem . . . . . . . . . . . . . . . . . . 33

4-1 LQ Regulator and Kalman Filter Interconnection . . . . . . . . 38

5-1 Regular Plate vs. Thick Plate (twice the thickness) . . . . . . . 40

$5-2$ Regular Plate vs. Two Plates $(9.875$ in apart $) \ldots \ldots . \ldots 41$

5-3 MIMO Controller Configuration . . . . . . . . . . . . . . 42

5-4 MIMO Controller Open Loop $G_{y u}$ Transfer Functions . . . . . . . 44

5-5 MIMO Controller Open Loop $G_{y w}$ Transfer Functions . . . . . . . . 45

$5-6$ MIMO Controller $G_{u y}$ Transfer Functions . . . . . . . . . 47

$5-7$ MIMO Controller Nichols Chart . . . . . . . . . . . . . . . . 48

$5-8 \quad$ MIMO Controller Performance . . . . . . . . . . . . . . . . . 49

$5-9$ Successive Loop Controller Configuration . . . . . . . . . . . 51

5-10 SLC Controller Bottom Plate Open Loop $G_{y u}$ and $G_{y w}$ Transfer Func-

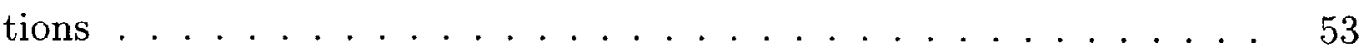

5-11 SLC Controller Top Plate Open Loop $G_{y u}$ and $G_{y w}$ Transfer Functions 53

5-12 SLC Controller Open Loop $G_{z w}$ Transfer Functions for Both Plates . 54 
5-13 SLC Controller $G_{u y}$ Transfer Functions . . . . . . . . . . 56

5-14 SLC Controller Nichols Chart . . . . . . . . . . . . . 57

5-15 Successive Loop Closure Controller Performance (Bottom Plate System

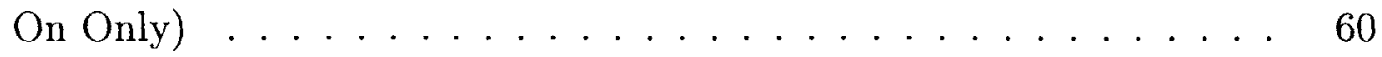

5-16 Successive Loop Closure Controller Performance (Both On) . . . . . 61

5-17 Interference Controller Configuration . . . . . . . . . . . . 62

5-18 Interference Controller Bottom Plate Open Loop $G_{y u}$ and $G_{y w}$ Transfer

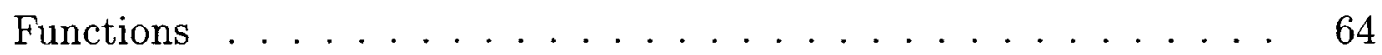

5-19 Interference Controller Top Plate Open Loop $G_{y u}$ and $G_{y w}$ Transfer

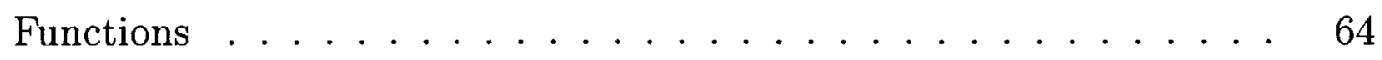

5-20 Interference Controller Open Loop $G_{z w}$ Transfer Functions for Both Plates . . . . . . . . . . . . . . . . 65

5-21 Interference Controller $G_{u y}$ Transfer Functions . . . . . . . . 67

$5-22$ Interference Controller Nichols Chart . . . . . . . . . . . 68

5-23 Interference Controller Performance (Top Plate System On Only) . . 71

5-24 Interference Controller Performance (Both On) . . . . . . . . 72

5-25 Diode Controller Configuration _.. . . . . . . . . . . 73

5-26 Diode Controller Bottom Plate Open Loop $G_{y u}$ Transfer Functions . 75

5-27 Diode Controller Bottom Plate Open Loop $G_{y w}$ Transfer Functions . 75

5-28 Diode Controller Top Plate Open Loop $G_{y u}$ and $G_{y w}$ Transfer Functions 76

5-29 Diode Controller Open Loop $G_{z w}$ Transfer Functions for Both Plates 76

5-30 Power Diode Controller $G_{u y}$ Transfer Functions . . . . . . . 78

5-31 Power Diode Controller Nichols Chart . . . . . . . . . . . . 79

5-32 Power Diode Controller Performance (Bottom Plate System On Only) 82

$5-33$ Power Diode Controller Performance (Both On) . . . . . . . . 83

5-34 Power Diode Controller Reverse Direction (Both On) $\ldots \ldots . . .84$

5-35 Single Layer Active Transmission Control . . . . . . . . . . . . 86 


\section{List of Tables}

2.1 Lengths of Test Chamber Sections and Spacers . . . . . . . . . . 23

5.1 MIMO Controller Performance Summary . . . . . . . . . . . 50

5.2 SLC Controller (Bottom Only) Performance Summary . . . . . . . 59

5.3 SLC Controller (Both On) Performance Summary . . . . . . . . . 59

5.4 Interference Controller (Top Only) Performance Summary . . . . . 70

5.5 Interference Controller (Both On) Performance Summary . . . . . . . 70

5.6 Power Diode Controller (Bottom Only) Performance Summary . . . . 81

5.7 Power Diode Controller (Both On) Performance Summary . . . . . 81

6.1 Test Configuration Performance Summary . . . . . . . . . . 88 


\section{Chapter 1}

\section{Introduction}

\subsection{Motivation}

During launch, payloads within the rocket fairing experience severe vibro-acoustic loads, which account for over $40 \%$ of first-day launch failures [1]. Failure types include but are not restricted to [2]:

- Malfunction of electronic and mechanical components in the vehicle

- Fatigue failure of internal components and supporting hardware

- Fatigue of lightweight exterior structures such as antennae

- Adverse environmental conditions for vehicle occupant

Payload fairings are typically designed to first meet structural requirements and then meet acoustic requirements [3]. The introduction of advanced composites, which possess greater stiffnesses and strengths than traditional metals such as aluminum, has led to the design of lighter structures. Although the mass savings are considerable, the move to thinner composite structures has led to greater vibro-acoustic problems due to their lower inertias. The goal of this thesis is to develop methodologies and technologies that will reduce the global acoustic field within a composite rocket fairing, thereby increasing the payloads' survivability without adding a prohibitive amount of mass. 
Modifying the acoustic field within the fairing is preferable to requiring more robust payload structures to solve the vibro-acoustic problem for two reasons. First, the time period over which the payloads experience the largest loads is very short compared to the life-cycle of most satellites. The primary source of the acoustic field is the fluctuating turbulence in the mixing region of the rocket-exhaust flow. Although noise is radiated in all directions, it is the greatest when directed perpendicularly to the vehicle's axis, as in the case of lift-off [2]. Therefore, it is preferable to design the fairing to decrease transmitted vibro-acoustic loads rather than to over-engineer the payload.

Second, while the cost and difficulty in proofing sensitive payload instruments against the vibro-acoustic loads may be prohibitive, the fairing presents a relatively inexpensive and straightforward structure to manipulate. Using proper techniques, the acoustic signature within the fairing can be reduced globally without drastically changing the basic design of the fairing. Using the fairing to reduce vibro-acoustic loads also negates the need to develop new certification codes for payloads, which can be both time-consuming and expensive.

\section{$1.2 \quad$ Background}

The vibro-acoustic problem is concerned with the net flow of acoustic energy into a control volume that defines the region of interest. It can be divided into two distinct and separate subproblems: reflection and transmission control. The goal of reflection control is to minimize the amount of reflected acoustic energy that originates from within the control volume and is reflected back at a material interface (in this case, the fairing/interior acoustic medium boundary). Conversely, the goal of transmission control is to minimize the amount of transmitted acoustic energy that originates from outside the control volume. The exterior acoustic energy induces structural motion, which then generates an acoustic field within the control volume. In either of the two subproblems, the goal is the same: the acoustic energy within the control volume is to be minimized. 
The minimization of the acoustic energy within the control volume is a difficult problem and may involve several methods of acoustic control. The methods of acoustic control can be separated into two distinct categories: active and passive control techniques. In some cases, the technique may address both reflection and transmission control; in other cases, just one of the two. Some of the techniques that are used in acoustic control are described below. Each technique has its advantages and disadvantages. However, it is likely that acoustic control systems will incorporate both active and passive techniques in the future.

\subsubsection{Active Control Techniques}

The two most popular types of active acoustic control techniques are Active Noise Control (ANC) and Active Structural Acoustic Control (ASAC). Both types of control techniques have been applied successfully in order to reduce acoustic energy within a control volume. However, the methods by which the energy is reduced are different. ASAC is the preferred choice in situations where the acoustic field is generated by a vibrating structure that can be readily controlled and where global attenuation is desired. In cases where the acoustic reduction is only desired in a local area or where it is undesirable to actuate on the vibrating structures, ANC is preferred. The two methods are described in greater detail in the following subsections.

\section{Active Noise Control (ANC)}

Active Noise Control has been used successfully in aircraft fuselages, noise cancelling headphones and in theaters. The idea behind Active Noise Control is to use acoustic sources to cancel out incoming acoustic disturbances. This technique, based on the concept of destructive interference, is highly dependent upon the placement of these

acoustic sources as well as their number $[4,5]$. Because the technique relies heavily on the phase of the noise and the canceling acoustic wave, its effectiveness is limited to situations where the noise is limited to a narrow acoustic band or is tonal in nature. Another complication is that in more complex geometries, there can be zones 
of acoustic magnification which require a large number of acoustic sources and sensors in order to ensure that the areas of acoustic magnification do not occur in areas of interest. Performance is best when the acoustic sources are placed near the origin of the disturbance [6]. However, physical constraints on the problem usually dictate how close the acoustic sources can be placed with respect to the origin of the noise.

\section{Active Structural Acoustic Control (ASAC)}

Active Structural Acoustic Control has been applied to many problems in sound transmission and structural sound radiation [3]. It is different than ANC in that it is the structure that is being controlled directly and not the acoustic field itself. Structural actuators can take on many forms, including shakers [7] and piezoelectric patches [8]. Global attenuation of the acoustic field can be attained using only a few actuators, whereas ANC requires many acoustic sources. Because the required number of ASAC actuators is less than that of ANC and because piezoelectric patches are smaller and lighter than speakers, ASAC can be used in situations where weight and volume are at a premium, as in the case of a payload fairing. However, ASAC is used primarily to address transmission control because it is difficult to match the impedance of the structure with that of the acoustic medium.

\subsubsection{Passive Control Techniques}

Passive control techniques attempt to modify the damping characteristics and stiffnesses of a structure without using active elements. Passive control techniques that have been considered for payload fairings include: adding non-structural mass [3], changing the fluid medium within the fairing [9] and adding acoustic blankets [10].

\section{Non-Structural Mass}

The addition of non-structural mass increases the insertion loss of the fairing and reduces the transmission of the acoustic loads into the payload bay. However, its effectiveness is frequency dependent, and it is most effective between the ring frequency 
of the the fairing and the critical frequency of the material. Below the ring frequency of the fairing, the addition of non-structural mass has very little effect, making it a poor choice for low frequency acoustic attenuation.

\section{Helium as a Fluid Medium}

Currently, the vibroacoustic disturbances are transmitted from the fairing to the payload through the fluid, air. Replacing air with a less dense fluid, such as helium, will reduce the structural-acoustic coupling. However, the payload fairings being used currently are not designed to be air-tight. As a rocket leaves the atmosphere, the pressure within the fairing matches the pressure outside it. Making the fairing air-tight would require sealing the entire payload compartment as well as possibly redesigning it to sustain the difference in pressure between the payload compartment and the outside environment. In addition, the lower density of helium would also cause a reduction in aerodynamic damping within the payload cavity, which may allow structural vibrations to become more severe. However, this side-effect is relatively minor because isolators are often used to reduce the amount of vibration being transmitted from the rocket to the payload.

\section{Acoustic Blankets}

The use of acoustic blankets is one of the more popular passive measures being utilized to reduce acoustic reflection within payload fairings. Other material systems used for the same purpose include sandwiched panels containing foam, syntactic, and balsa wood cores. The amount of acoustic absorption is dependent upon the material, thickness and excitation frequency. Typical thicknesses for the acoustic blankets range from two to four inches, which correspond to a peak absorption coefficient between 300 and $500 \mathrm{~Hz}$. At low frequencies, the thickness of the acoustic blankets is small compared to the acoustic wavelength, such that there is a wide disparity between the impedances of the fluid and the acoustic blankets. The impedance mismatch makes it difficult for the fluid to transmit its acoustic energy to the acoustic blankets, and the absorption coefficient is small. The same is true at very high frequencies, where 
the absorption coefficient is proportional to $1 / f^{5}[3]$.

\subsection{Objective}

This thesis has two objectives: to develop a theoretical framework for multi-layer Active Structural Acoustic Control (ASAC) and to demonstrate through experimentation the merits of such a system. The thesis will focus exclusively on the vibroacoustic subproblem of transmission control. The performance of using single-layer control and dual-layer control will be compared, and possible research paths for future ASAC development will be suggested.

The project will focus solely on transmission control because work done in the past $[11,12]$ suggests that the large impedance mismatch between structural materials and air prevents effective reflection control using traditional ASAC methods. Any reflection control gains using ASAC methods were offset by the diminished effectiveness of transmission control, and project constraints preclude the use of traditional ANC techniques such as noise-canceling speakers within payload fairings. Future research may yield technologies that solve the reflection control problem, and hopefully, the multi-layer approach will provide the framework to incorporate both transmission and reflection control into a single system.

\subsection{Previous Work}

The previous work done at MIT has advanced Active Structural Acoustic Control methodology significantly and serves as the basis for this thesis. Roger Glaese developed the idea of trying to match the impedance of the controller with that of the fluid. Because only the impedances at the material boundaries were considered, a local model of the system behavior was used to design a compensator. The fact that the compensator only had a local model of the system meant that the resulting compensator could not rely on performance measurements in the acoustic field or on direct measurements of the acoustic disturbances. As a consequence, the controller 
was suboptimal. However, Glaese was able to demonstrate the viability of using feedback techniques for an ASAC system, and he stressed the importance of the idea of impedance as a way of maximizing energy flow away from a system [1].

Koji Asari attempted to address both reflection and transmission control. Two types of controllers were used: a sensitivity weighted LQG controller and an acoustic impedance matching controller. The structural modes were targeted using a sheet of polyvinylidene fluoride (PVDF) as a strain sensor and a patch of piezoceramic wafer as an actuator. The acoustic modes were targeted using a speaker as an actuator and a microphone as a sensor. For both structural and acoustic modes, the acoustic field within the chamber was attenuated significantly [11]. However, implementing an ANC system within a payload system is impractical due to weight and space restrictions, such that another solution for reflection control must be found.

Robert Pascal constructed a finite element model for the acoustic test chamber using ANSYS. Piezoelectric actuators, microphones, accelerometers, speakers, strain gauges were modeled within the finite element model, which allowed Pascal to verify the relative merits of each sensor/actuator and their locations. Pascal had also tried to use multiple piezoelectric patches as sensuators, which can exert a force and sense the amount of deflection at the same time. However, the distributed sensuator was not successfully implemented because it proved to be very sensitive to unmodeled but present manufacturing (slightly warped plate within design tolerances) and boundary condition uncertainties (edges boundary conditions are a combination of clamped and pinned idealizations). The fact that the piezoelectric material properties are very sensitive to their environment and must be known precisely made the problem that much more difficult. However, the finite element model that Pascal had constructed proved to be extremely useful for identifying the structural and acoustic modes [13].

Carlos Gutiérrez developed a structural acoustic diode that was designed to attenuate the transmission of acoustic energy from the outside to the inside while allowing acoustic energy to flow more easily from the inside to the outside. The results from the acoustic power diode's implementation were worse than when separate controllers were used to target the acoustic and structural modes independently. The two control 
loops used in the diode's implementation (one as the usual force feedback upon the structure to reduce acoustic transmission, the other as an impedance match between the fluid and the plate) occasionally offered contradictory commands to the plate. As a consequence, the plate was not able to perform either task optimally, and its overall performance suffered [12].

\subsection{Thesis Outline}

This thesis presents the results of using multi-layer active structural control to reduce the amount of energy flowing from one side of the test chamber to the other. The two sides of the test chamber represent two distinct and separate volumes: the volume representing the "inside" of the launch fairing and the volume representing the "outside" of the launch fairing that represents the source of the structural-acoustic disturbance. Two structural layers and four different controller configurations were used in the multi-layer configuration to determine which design methodology was most effective in achieving broadband reductions in the transmission of acoustic energy across the test chamber.

Chapter 2 describes the various test chamber configurations used during the experiments. A single layer configuration was used to provide the baseline metric to which all the dual layer systems could be compared to. Two dual-layer configurations are also presented. The first configuration had an open ended "interior" cavity while the second configuration was sealed with an end cap at that end. The first configuration was used for most of the experimental work as three of the four controller configurations were designed for it. However, the second configuration was necessary because the fourth controller (Power Diode) configuration required a disturbance source at both the top and the bottom of the test chamber to verify that it was working properly. The four controller types are described fully in Chapter 5. The dimensions of the test chamber and the structural plates are listed, and the types of electronics and computational hardware used to support the experiments are also described.

Chapter 3 describes how state-space models that are used to develop controllers 
are derived. The measurement model methodology was used exclusively to obtain plant descriptions. Transfer functions between the disturbance sources, actuators and sensors were taken experimentally, and state-space representations of the model were obtained using the Frequency Domain Observability Range Space Extraction (FORSE) algorithm developed by Jacques. The state-space models were then used to develop controllers that were implemented and tested within the test chamber.

Chapter 4 describes the methodology that was used to develop the controllers. The Linear Quadratic Gaussian (LQG) Controller formulation was used to design all the controllers used in the experiments. The LQG Controller is broken down to its two components: the Kalman filter that served as a state estimator and a Linear Quadratic (LQ) Regulator that determines actuator inputs. The series interconnection of the Kalman filter and the LQ Regulator is also described.

Chapter 5 describes how each of the four controller types are developed and presents the results achieved by each controller type. The four types of controllers used were the Multi-Input Multi-Output (MIMO) Controller, the Successive Loop Closure (SLC) Controller, the Interference Controller and the Power Diode Controller. Open loop transfer functions and controller transfer functions are presented, and the closed loop transfer functions are compared to their open loop counterparts to determine broadband performance. Advantages and disadvantages of each controller are discussed, and narrowband and broadband performance metrics are given for all four controller types.

Chapter 6 summarizes the results presented in Chapter 5 and presents suggestions for future research. 


\section{Chapter 2}

\section{Experimental Setup}

\subsection{Test Chamber}

\subsubsection{Dimensions}

A picture of the test chamber is shown in Figure 2-1. Most of the experiments were conducted without the top end section, for which the test chamber's length was 40.38 in (including two wooden spacers). The top end section was only used when implementing the Power Diode, which increased the test chamber's overall length to 48.38 in (including two wooden spacers). The test chamber is made of cast iron, and its diameter is twelve inches. The iron tube is subdivided into three sections, and their dimensions are given in Table 2.1.

Table 2.1: Lengths of Test Chamber Sections and Spacers

\begin{tabular}{l||r}
\hline Section & Length (in) \\
\hline \hline Top End Section & 8.00 \\
Upper Section & 15.00 \\
Middle Section & 8.71 \\
Upper Ring Spacer & 0.46 \\
Lower Ring Spacer & 0.71 \\
Bottom End Section & 15.50 \\
\hline Total Length & 48.38 \\
\hline
\end{tabular}




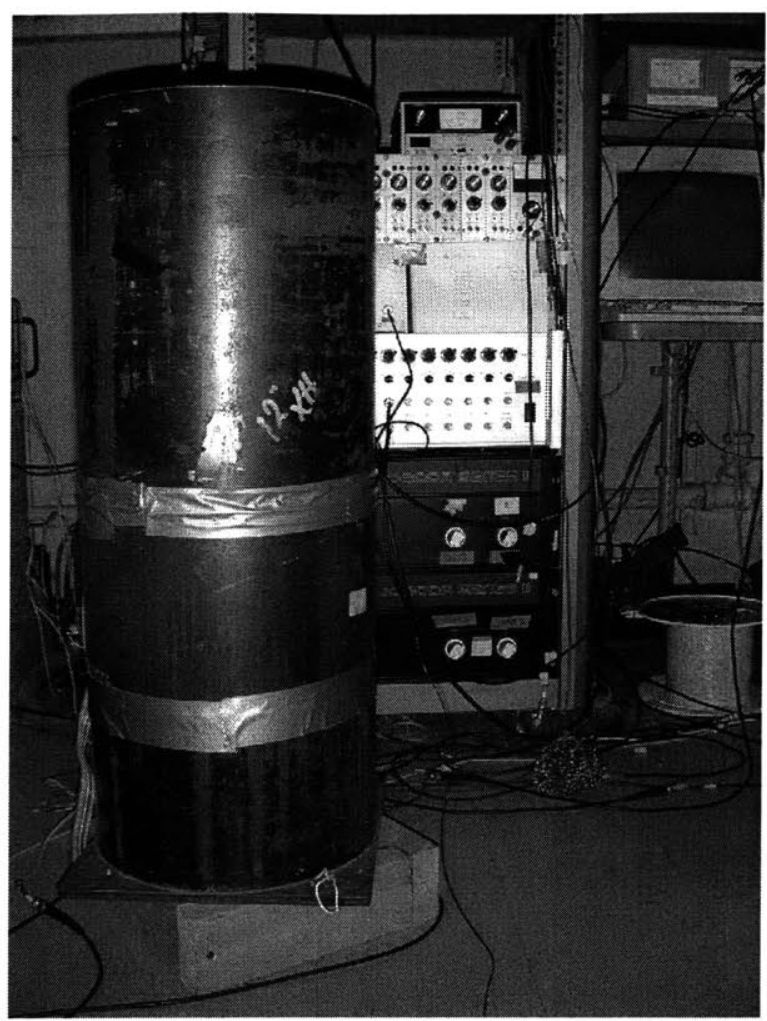

Figure 2-1: Test Chamber in Single Layer Configuration

\subsubsection{Excitation Sources}

A pair of ten inch diameter sub-woofers served as the disturbance sources. Each of the two speakers was located in one of the end sections of the chamber. All test chamber configurations included the bottom speaker, and only the Power Diode configuration made use of the top speaker. The top speaker was used to affirm that the acoustic energy was only allowed to pass the Power Diode in one direction. Therefore, for all the experiments not related to the Power Diode, the top end section, including the top disturbance speaker, was removed. The speakers were set to generate broadband noise over the $20-1000 \mathrm{~Hz}$ range, which is representative of the type of acoustic excitation that payloads are subjected to during launch.

\subsubsection{Test Chamber Configurations}

Two main test chamber configurations were used during the experiments: a one plate system and a two plate system. Figure 2-2 shows the schematic of the one plate 


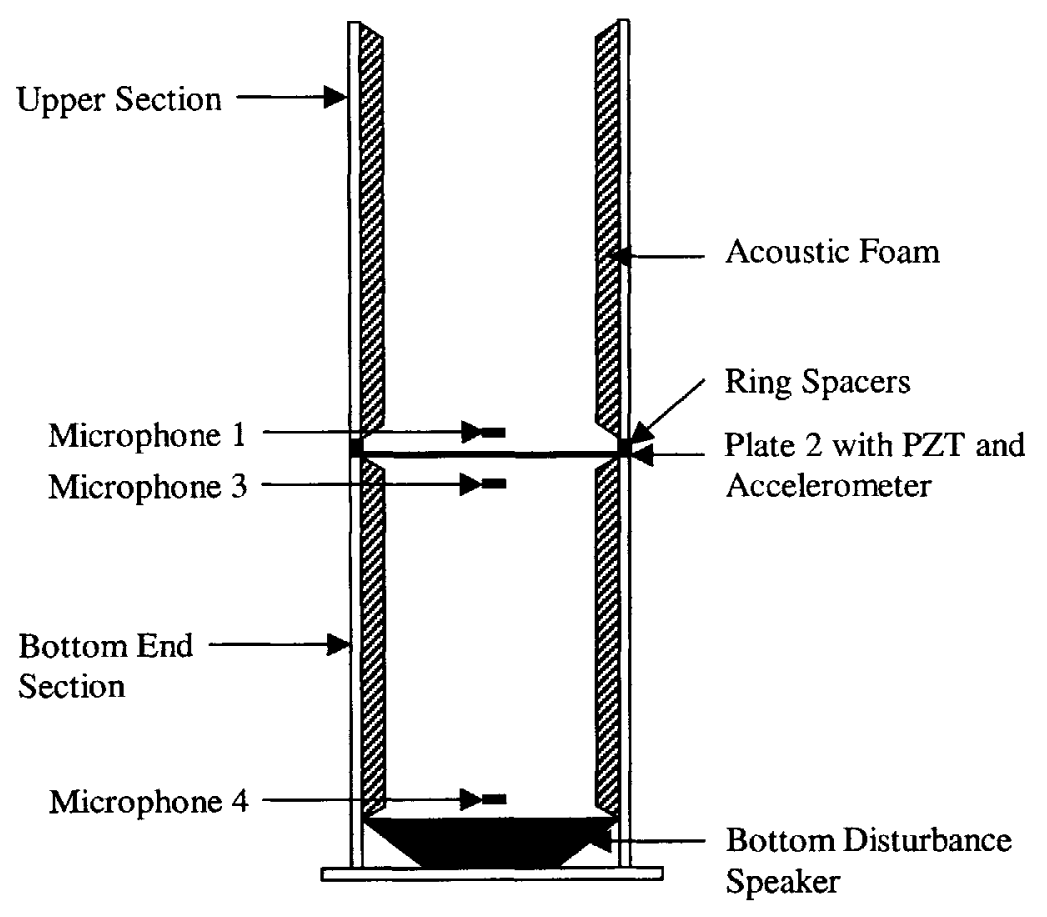

Figure 2-2: Single Layer Chamber Configuration

system, which was used to determine how the transmission of vibro-acoustic energy is affected by doubling the mass and thickness of a plate. The one plate system also provided a baseline as to how effective actively controlling a single plate was in reducing the transmission of vibro-acoustic energy compared to two plate systems.

Figure 2-3 shows the schematic of the two plate system. Plate 2 is located at the same spot in both the single and dual plate configurations. In the two plate configuration, Plate 1 is set above Plate 2 at three different distances: $0.046,1.16$ and $9.88 \mathrm{in}$. These distances were achieved using two wooden ring spacers that have thicknesses of 0.46 and $0.70 \mathrm{in}$, and an iron section of the pipe that has a length of 8.71 in. The Power Diode configuration shown in Figure 2-4 uses the two plate system where the plates are set 9.88 in apart and the top end section of the test chamber is added. In all test chamber configurations, acoustic foam was placed along the inner surface of the Bottom End Section and of Upper Section to minimize the excitation of the non-axisymmetric modes that resulted from an imperfect test chamber. 


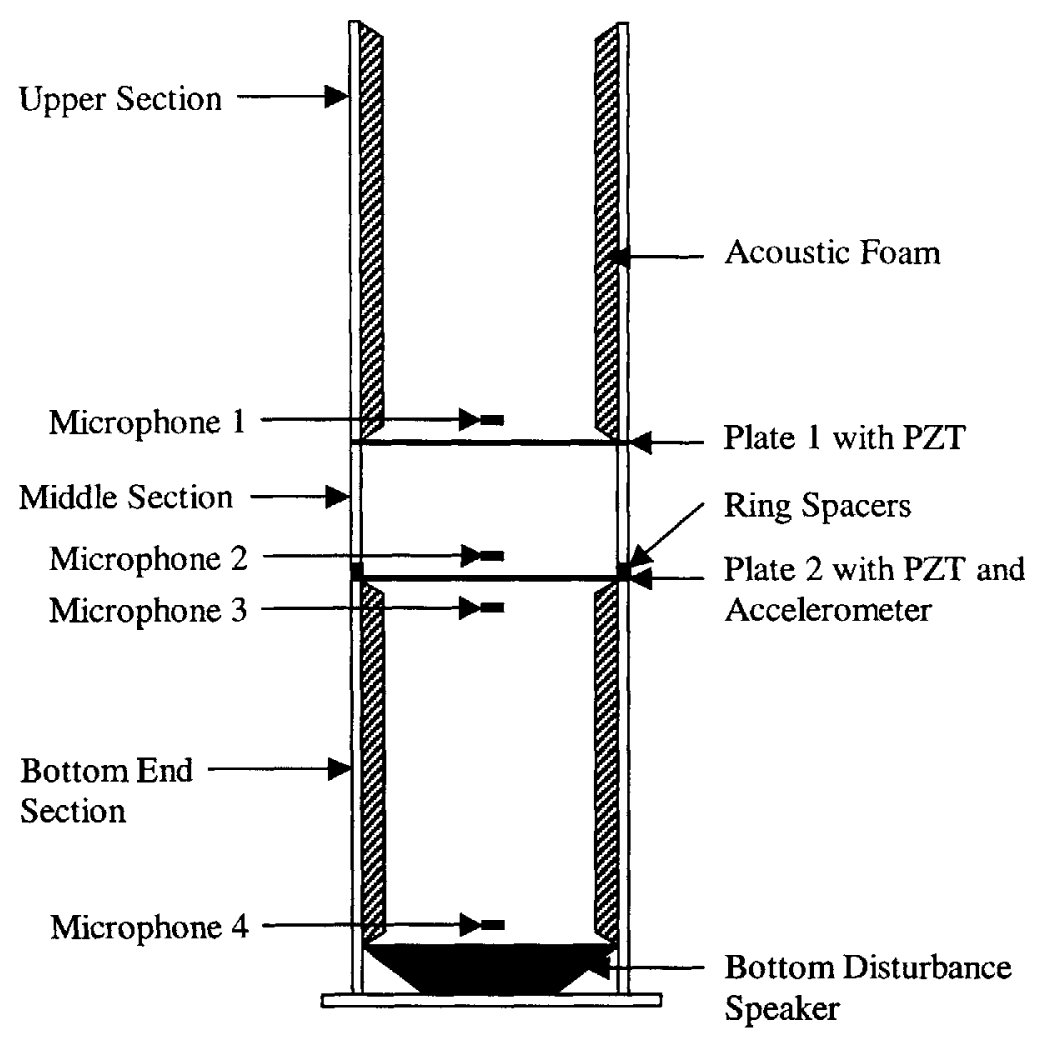

Figure 2-3: Dual Layer Chamber Configuration

\subsubsection{Structural Plates}

All of the plates are made of aluminum and are twelve inches in diameter. One plate is $\frac{1}{16}$ in thick while all the others are $\frac{1}{32}$ in thick. The thicker plate was only used to compare the acoustic transmission characteristics when the mass and thickness of the structural plate were doubled without using any active control. Therefore, the thicker plate did not have a PZT actuator affixed to it. PZT patches were affixed to the two regular plates that were $\frac{1}{32}$ in thick, and an accelerometer was affixed to one of these two plates (on the opposite side as the PZT patch). The PZT patches were affixed to the aluminum plates using a mixture of thinned-out five minute epoxy and conductive epoxy. A glob of conductive epoxy was placed on the center of each of the PZT patches to ensure that the lower surfaces of the PZT actuators and the upper surfaces of the aluminum plates had good electrical connections. Thinned-out five minute epoxy was dabbed onto the area that did was not covered by the conductive epoxy to provide a strong bond between the aluminum plates and the PZT actuators. 


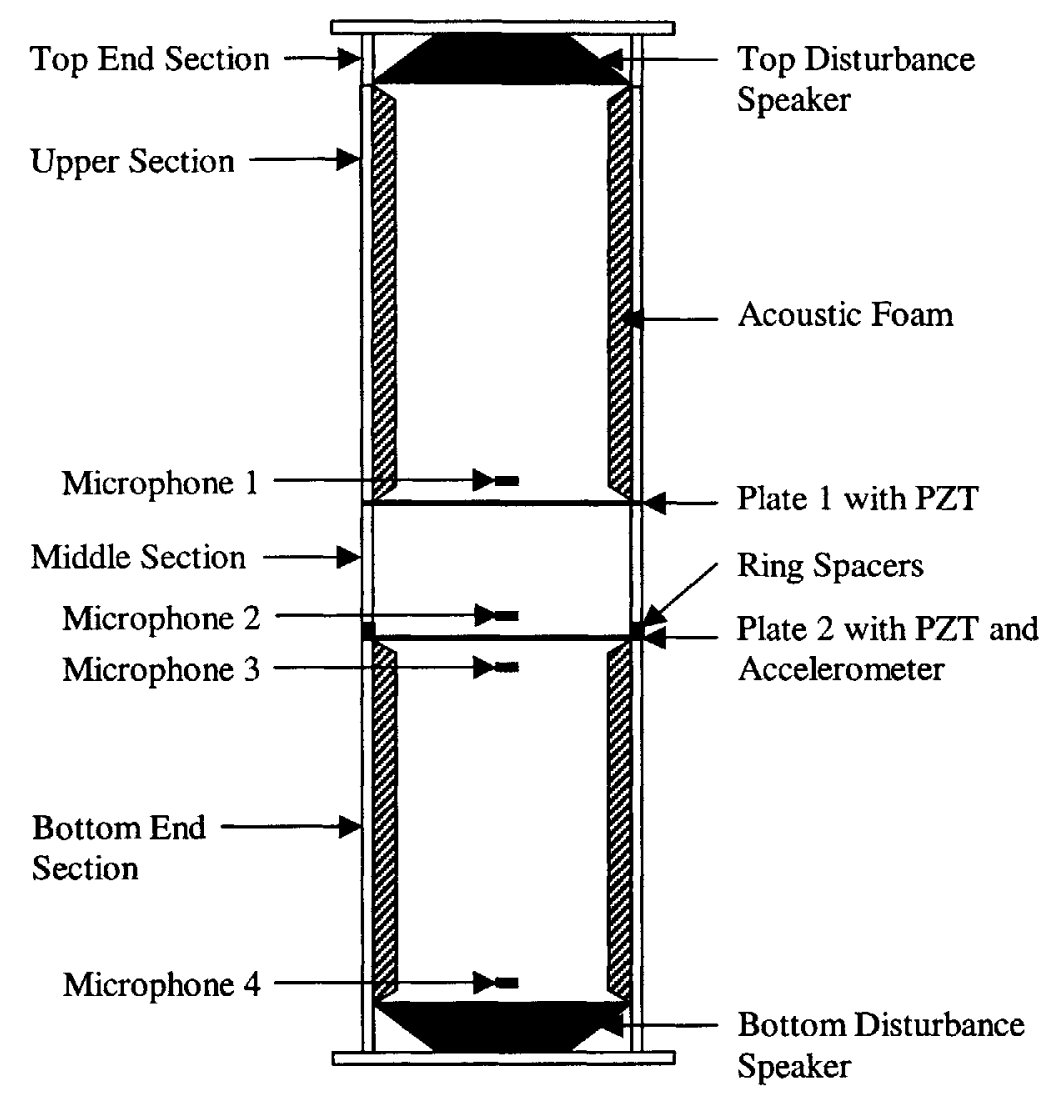

Figure 2-4: Diode Chamber Configuration

The accelerometer was also affixed using five minute epoxy. The plate that had both a PZT patch and an accelerometer was designated as Plate 2, and the plate that only had a PZT patch was designed as Plate 1. Both plates were mounted in the test chamber with the piezo facing upward. A picture of Plate 1 is shown in Figure 2-5.

\section{$2.2 \quad$ Electronics}

\subsubsection{Amplifiers}

The test chamber provided the environment necessary to conduct structural-acoustic experiments, but electronics and computational power were required to make the active transmission control possible. Two Crown DC-300A Series II power amplifiers, with two channels each, were used to provide power to the two disturbance speakers and to the two PZT actuators. The amplifiers provided $305 \mathrm{~W}$ to each channel. A 


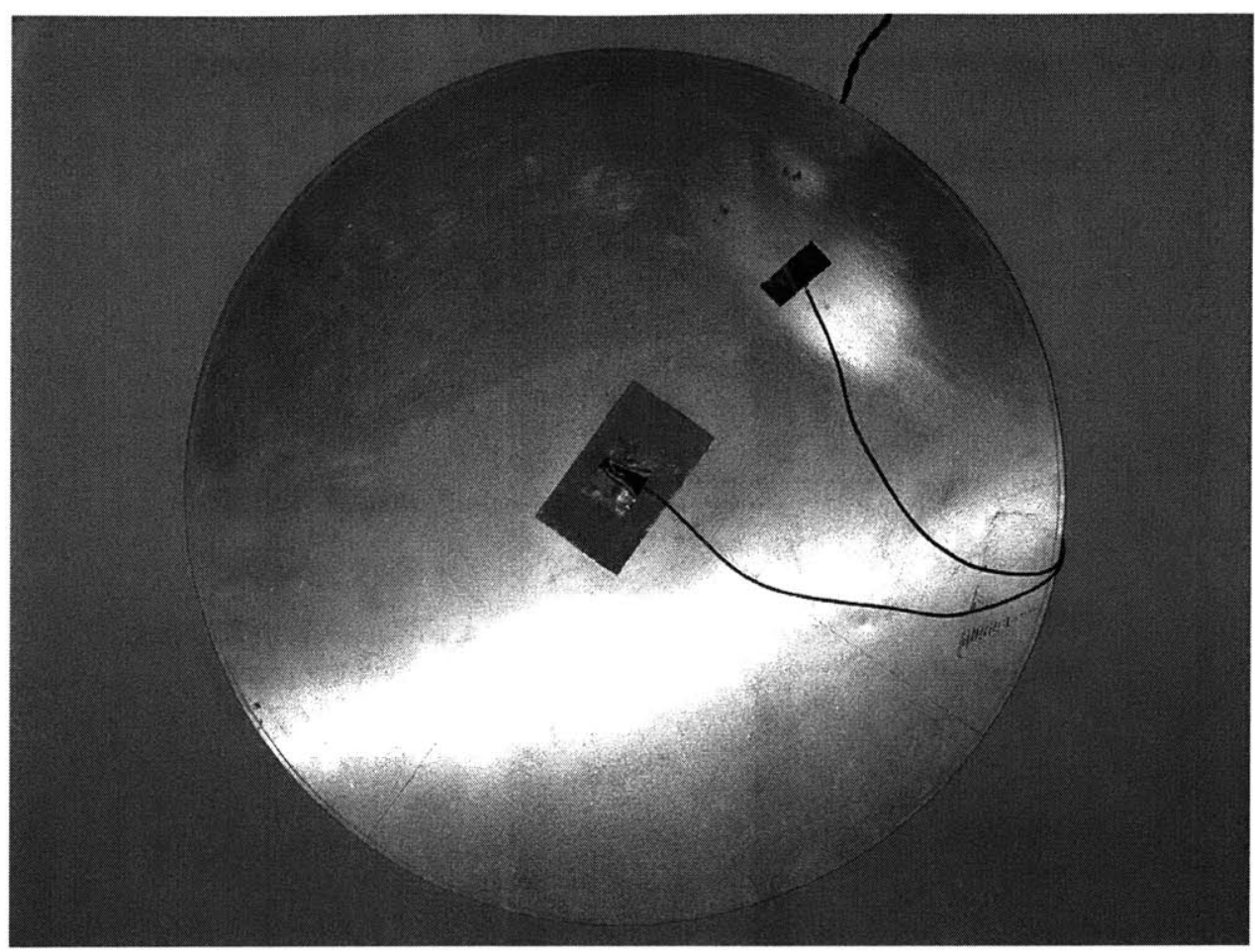

Figure 2-5: Structural Plate with PZT Patch

block diagram of the electrical system is shown in Figure 2-6.

\subsubsection{Measurement Devices}

Microphones and an accelerometer were used to measure the pressure of the acoustic field along the test chamber. An ENDEVCO charge amplifier was used as the signal conditioner for the accelerometer, and a 3-10 V VDC power supply was used as a power source for the electret condenser microphones. Conditioners with variable gain were also used for the microphones. Each microphone was different and the signals that each one produced varied in magnitude. The gains of the conditioners were set to eliminate these differences. Several microphones were set to measure the pressure at the same point, and the conditioner gains were set until the amplitudes of their output voltages were identical.

Besides being used to calibrate the microphones, the conditioner gains were important in that the conditioner output voltage range should match the input range of the analog to digital (A/D) converter board of the real time computer. Because the 


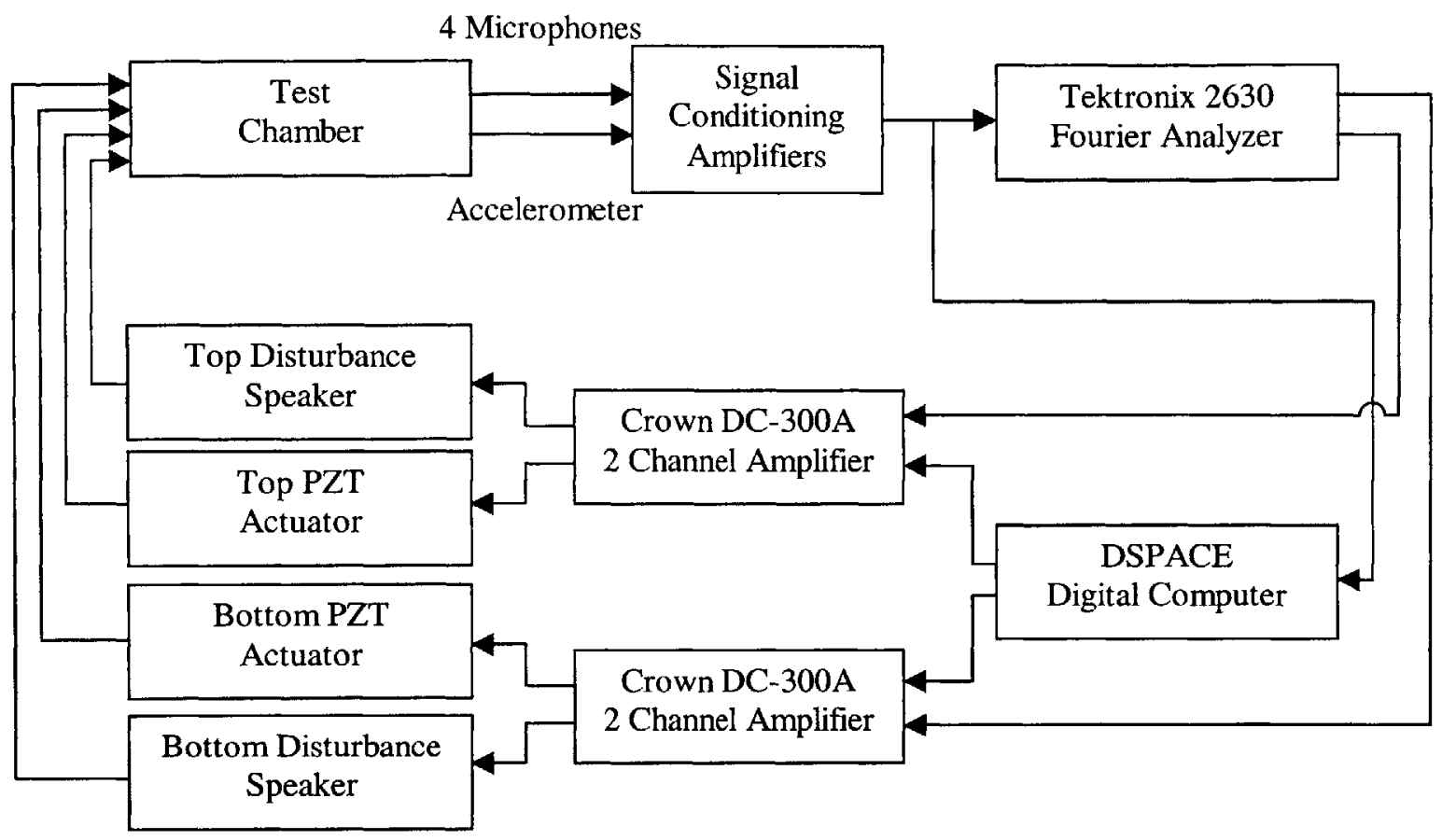

Figure 2-6: Block Diagram of the Experimental Setup

analog signals are resolved digitally, the resolution of the A/D converter was fixed over its $\pm 10 \mathrm{~V}$ range. By matching the magnitudes of the conditioned signal to the input range of the A/D converter, the quantization error was minimized.

\subsubsection{Function Generator}

A Tektronix 2630 Fourier Analyzer was used to generate the band-limited white noise that was used to excite the system. The unit had one output and four inputs, so that multiple responses could be recorded for a given excitation. The multiple inputs of the unit were useful because they allowed transfer functions to be obtained two at a time. The output was directly fed into one of the inputs, so that, hypothetically, three transfer functions could be taken at once. However, the software interface limited the number of transfer functions that could be taken to two. The transfer functions taken were between the various inputs (disturbance speakers and PZT patches) and the various outputs (microphones and accelerometer). The transfer functions were then used to create a state-space model of the plant, so that controllers could be designed and implemented. The Tektronix 2630 Fourier Analyzer was also used to 
take transfer functions while the controllers were running. Comparing the transfer functions when the controllers was running to when they were not gave an indication of how well the controllers were performing.

\subsubsection{Real Time Computer}

The real time computer used to implement the controllers during experimentation was located on an expansion board that had been installed into a Pentium PC. The dSPACE expansion board had a PowerPC 750 processor running at $480 \mathrm{MHz}$ that was dedicated to real time processing. This performance translated to being able to run two controllers with up to fifty-five states each at $5 \mathrm{kHz}$ simultaneously.

The expansion board that contained the real time processor was connected to an input and to an output expansion board that were also installed in the host computer. The input and output expansion boards were then connected to rack-mounted input and output boards that had thirty-two BNC connectors each. Coaxial cables were used to connect the inputs and outputs of the real time computer to the microphones and accelerometer, and to the PZT actuators, respectively.

MATLAB was used to design the controllers, and software that accompanied the dSPACE system was used to compile and down-load the controllers onto the real time processor. ControlDesk, which was also bundled with the dSPACE system, was used to change controller parameters (such as gains) interactively once it was down-loaded to the processor, which saved a lot of time because the controller did not have to be recompiled every time controller parameters were changed. 


\section{Chapter 3}

\section{Structural-Acoustic Modeling}

\subsection{Measurement Model}

The choice of using a measurement-based model instead of a finite element model was based on the results obtained by Robert Pascal [13] and Koji Asari [11]. Although useful in offering insight toward the physical behavior of the test chamber, the finite element model could not produce a model with enough accuracy such that an effective structural controller could be designed. In contrast, the measurement-based method is able to produce an accurate model of the system that include external influences that may or may not be captured using an idealized model. Therefore, all controllers were designed using measurement models. However, there are three main disadvantages of measurement models that a user must be aware of.

First, all physical intuition into the dynamics of the system is lost because the model is obtained by minimizing the difference between two curves: the transfer function of a state-space realization and the transfer function of the measured model. Second, the types of external influences that the measurement model includes is dependent upon what the designer chooses to include in the transfer function matrix, which may affect compensator performance, and some input-output relationships may not even be quantifiable because the performance variables and disturbance sources are prohibitively difficult or impossible to measure. Third, although the frequency, damping and amplitude of the system poles can be specified explicitly, zeros are a lot 
more difficult to characterize. As a consequence, non-minimum phase zeros can occur in the model depending on the order in which the model states were eliminated or at what point the model is tuned. The presence of non-minimum phase zeros affects the bandwidth of the controller and, ultimately, compensator performance.

\subsubsection{Transfer Function Matrix}

The measurement model requires the open loop system response excited by a known input. In the case of the test chamber, band limited white noise was used to excite all the input channels (actuators and disturbance sources), and measurements were taken at all the output channels (sensor and performance measurements). The transfer functions taken between each input and output can be arranged in a four block transfer function matrix as shown in Figure 3-1. $\mathrm{G}_{\mathrm{zw}}$ represents the transfer function block from the disturbance sources to the performance measurements. $G_{z u}$ represents the transfer function block from the control actuators to the performance measurements. $\mathrm{G}_{\mathrm{yw}}$ represents the transfer function block from the disturbance sources to the feedback sensors, and $\mathrm{G}_{\mathrm{yu}}$ represents the transfer function block from the control actuators to the feedback sensors.

\subsubsection{State-Space Model}

Once the transfer function matrix was constructed, a state-space model was obtained using a program that uses the Frequency Domain Observability Range Space Extraction (FORSE) algorithm. The FORSE algorithm, developed by Jacques [14], creates a state-space realization that most closely resembles the measurement model transfer functions. It is based on Liu's Observability Range Space Extraction (ORSE) algorithm [15] but is extended from arbitrary real signals to complex signals.

The procedure used in obtaining the state-space model is as follows. A large number of states is assigned to parameterize the model. The large number of states leads to greater model fidelity, but the resulting large matrix equations require too much time to compute to be used in real time applications. Instead, the model 


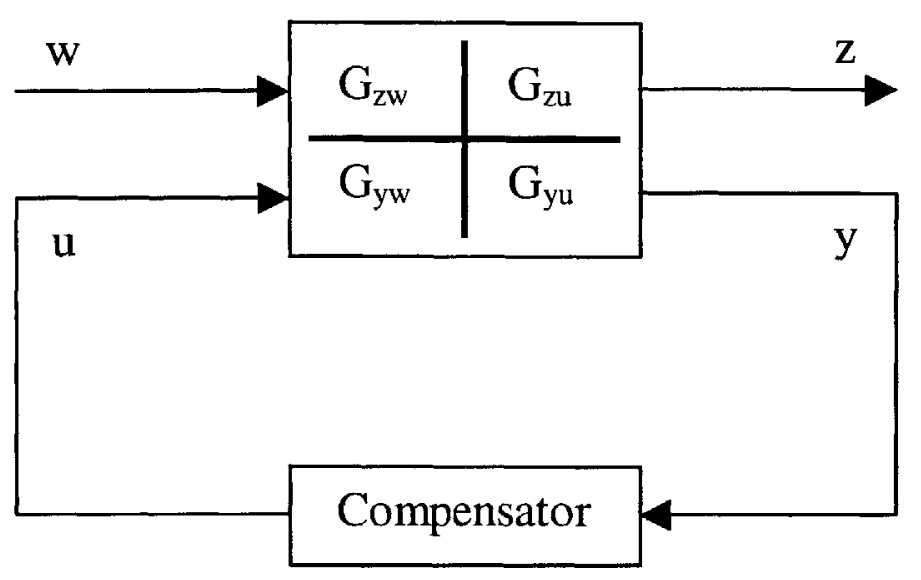

Figure 3-1: The Four Block Problem

is reduced by removing the states that are the least important. The hierarchy of state importance is determined by transforming the original state-space system such that the observability and controllability grammians are the same as the diagonal matrix of the Hankel singular values. States with the lowest Hankel singular values are eliminated, and the model is retuned to readjust and to reoptimize the model. The model tuning uses non-linear optimization algorithms that are applied to system identification. The FORSE algorithm uses the Levenberg-Marquardt algorithm [16], which is designed to solve the non-linear least squares problem where a cost function that is the sum of the quadratic errors is minimized. The reduction and tuning process is repeated until an accurate model that is small enough is obtained. 


\section{Chapter 4}

\section{Linear Quadratic Gaussian Controller}

The Linear Quadratic Gaussian (LQG) or $\mathcal{H}_{2}$ controller assumes linear dynamics, a quadratic cost function and Gaussian noise. Unlike a Linear Quadratic Regulator (LQR) that uses state feedback, the LQG controller does not have access to the state vector and requires a state estimator. The additional requirement of a state vector affects LQG controller performance compared to the LQR. The LQR is guaranteed to have at least $60^{\circ}$ phase margin and a $6 \mathrm{~dB}$ gain margin [17], but the LQG controller has no performance guarantees. However, the LQG design methodology was required for the structural-acoustic problem posed in the thesis because the state-space realization is based purely on the experimentally determined transfer functions. The resulting state-space description is only an optimized curve-fit and does not have any physical meaning. Therefore, an estimator has to be used to bridge the gap between the signals obtained from the sensors and a state regulator, and a LQG controller provides a good solution to the control problem because it provides optimal solutions to both the estimation and state-feedback problems.

The limitations, on which problems the LQG controller is designed to solve, are strict. The most important constraint is that the dynamics have to be linear. For physical systems in general, system dynamics are nonlinear. The LQG controller methodologies are still applied to some of these problems, but the systems have to be 
linearized about predetermined operating points. This is also true in the structuralacoustic problem presented in this thesis where the dynamic pressure of the fluid and the velocity of the plate are related by a non-linear relationship. Therefore, controller performance will deteriorate or the controller will become unstable when then system starts behaving in a sufficiently non-linear fashion.

A second problem that affects how well LQG controllers operate in real systems is the nature and characterization of the disturbance source. Not all disturbance sources are Gaussian nor are all random processes ergodic. Ergodicity is difficult to prove and is often assumed, and the central limit theorem of statistics is often invoked in many engineering applications where noise is frequently due to a superposition of many small contributions, which tends toward a Gaussian distribution [18]. These assumptions and simplifications are hard to prove, but they reduce an unmanageable problem to a form that can be solved using known solution techniques and have proven to be useful in addressing many real world problems. These assumptions have been made in this structural-acoustic problem, although care was taken to verify that these assumptions were not violated grossly. In particular, the plant characteristics (transfer functions) were assessed periodically and compared with the original values to ensure that the process was stationary (a condition for ergodicity).

\subsection{LQG Controller Formulation}

The state-space description of the LQG problem formulation can be written as

$$
\begin{aligned}
\dot{\boldsymbol{x}} & =A \boldsymbol{x}+B_{1} \boldsymbol{w}+B_{2} \boldsymbol{u} \\
\boldsymbol{z} & =C_{1} \boldsymbol{x}+D_{11} \boldsymbol{w}+D_{12} \boldsymbol{u} \\
\boldsymbol{y} & =C_{2} \boldsymbol{x}+D_{21} \boldsymbol{w}+D_{22} \boldsymbol{u}
\end{aligned}
$$

and the quadratic cost function to be minimized is

$$
J_{L Q G}=\int_{0}^{\infty}\left(\boldsymbol{x}^{T} Q \boldsymbol{x}+\boldsymbol{u}^{T} R \boldsymbol{u}+2 \boldsymbol{x}^{T} N \boldsymbol{u}\right) \mathrm{d} t
$$


where $\boldsymbol{x}, \boldsymbol{w}, \boldsymbol{u}, \boldsymbol{y}$ and $\boldsymbol{z}$ are the state, disturbance or noise, control, output and performance variable vectors respectively. In all well-posed control problems $D_{22}=0$, or else the problem formulation would be singular. This problem can be solved by redifining $\boldsymbol{y}_{\text {new }}=\boldsymbol{y}-D_{22} \boldsymbol{u}$. For the controller design done for this thesis, $D_{22}=0$ was enforced when obtaining a state-space realization using the FORSE algorithm. The cross-coupling penalty matrix, $N$, was set to the null matrix because an explicit relationship between the state and the control did not exist. The modified equations used in controller design are:

$$
\begin{aligned}
\dot{\boldsymbol{x}} & =A \boldsymbol{x}+B_{1} \boldsymbol{w}+B_{2} \boldsymbol{u} \\
\boldsymbol{z} & =C_{1} \boldsymbol{x}+D_{11} \boldsymbol{w}+D_{12} \boldsymbol{u} \\
\boldsymbol{y} & =C_{2} \boldsymbol{x}+D_{21} \boldsymbol{w} \\
J_{L Q G} & =\int_{0}^{\infty}\left(\boldsymbol{x}^{T} Q \boldsymbol{x}+\boldsymbol{u}^{T} R \boldsymbol{u}\right) \mathrm{d} t
\end{aligned}
$$

The control gain, $K$, and the Kalman filter gain, $L$, can be derived independently by solving two algebraic Riccati equations (AREs) for $P$ and $S$. The two AREs are

$$
\begin{gathered}
A^{T} P+P A-P B_{2} R^{-1} B_{2}^{T} P+Q=0 \\
A S+S A^{T}-S C_{2}^{T} V^{-1} C_{2} S+B_{1} W B_{1}^{T}=0
\end{gathered}
$$

and the gains are given by

$$
\begin{gathered}
K=R^{-1} B_{2}^{T} P \\
L=S C_{2}^{T} V^{-1}
\end{gathered}
$$

$W$ and $V$ are the process and sensor noise intensities respectively. The equations for the compensator are given by

$$
\begin{aligned}
\dot{\hat{\boldsymbol{x}}} & =\left(A-B_{2} K-L C_{2}\right) \hat{\boldsymbol{x}}+L \boldsymbol{y} \\
\boldsymbol{u} & =-K \hat{\boldsymbol{x}}
\end{aligned}
$$




\subsection{LQR and Kalman Filter Interconnection}

MATLAB Controls Toolbox was used to design and implement the LQG controller. However, MATLAB does not have a function that directly determines the structure of the compensator. Instead the compensator was made from an interconnection of a LQ Regulator and a Kalman Filter. The algebraic Ricatti equations to be solved are still given by equations 4.5 and 4.6 , but the equations for the LQ Regulator and for the Kalman Filter are different.

The equations for the Kalman filter is

$$
\begin{aligned}
\dot{\hat{\boldsymbol{x}}} & =A \hat{\boldsymbol{x}}+B_{1} \boldsymbol{w}+B_{2} \boldsymbol{u}+L\left(\boldsymbol{y}-C_{2} \hat{\boldsymbol{x}}\right) \\
\boldsymbol{y} & =C_{2} \hat{\boldsymbol{x}}+\boldsymbol{v}
\end{aligned}
$$

and the equations for the LQ Regulator is

$$
\begin{aligned}
\dot{\boldsymbol{x}} & =A \boldsymbol{x}+B_{1} \boldsymbol{w}+B_{2} \boldsymbol{u} \\
\boldsymbol{u} & =-K \boldsymbol{x}
\end{aligned}
$$

The $\boldsymbol{x}$ in the LQ Regulator equations is the $\boldsymbol{y}$ of the Kalman Filter equations. The gains $K$ and $L$ are given by equations 4.9 and 4.10 . Figure $4-1$ shows a schematic of the interconnected system.

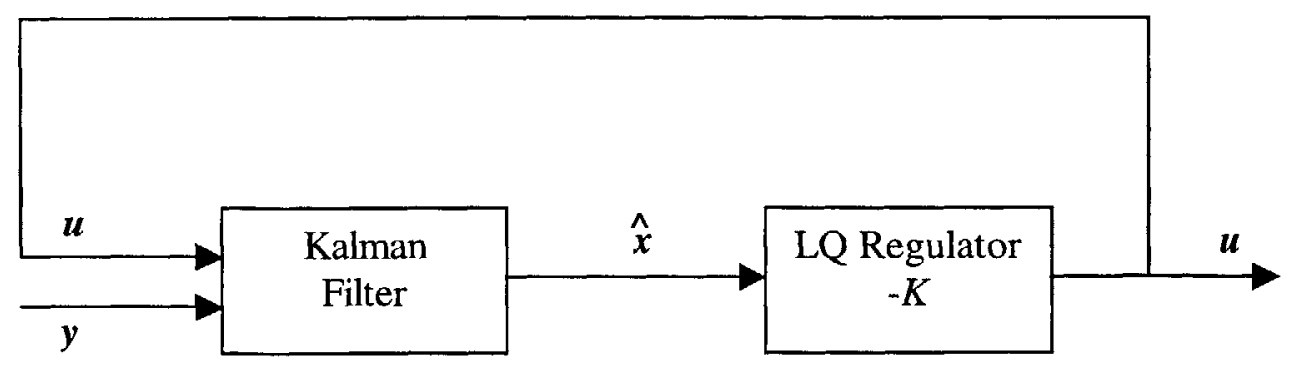

Figure 4-1: LQ Regulator and Kalman Filter Interconnection 


\section{Chapter 5}

\section{Experimental Results}

\subsection{Passive Configuration Performance}

The Single Layer Chamber Configuration and the Dual Layer Chamber Configuration shown in Figure 2-2 and Figure 2-3, respectively, were used to determine the effects of modifying characteristics of the structural layer. Figure 5-1 shows the transfer functions taken before and after plate 2 (plate 1 not present) was replaced with a plate twice its thickness and mass. The transfer function taken with the thicker plate in the system represented a $0.86 \mathrm{~dB}$ broadband reduction from 40 to $1000 \mathrm{~Hz}$ compared to the thinner, original plate. The frequency ranges of $40-90 \mathrm{~Hz}$ and $220-$ $1000 \mathrm{~Hz}$ experienced the greatest amounts of the attenuation relative to the original values, $14 \mathrm{~dB}$ and $9 \mathrm{~dB}$ respectively. However, these reductions occurred over ranges that had the smallest responses, so that their reductions were largely canceled by the 100-200 $\mathrm{Hz}$ range, where the response of the system was much larger.

Figure 5-2 shows the transfer functions taken before and after plate 1 was added to the system. The combined weights of the two plates is comparable to that of the thicker plate. At first glance, the reduction in the transfer function of the two plate system does not seem to be as large as the reduction obtained when using a thicker plate. Although the response from $40-90 \mathrm{~Hz}$ was only reduced by about $0.48 \mathrm{~dB}$ and the response from $220-1000 \mathrm{~Hz}$ reduced by a comparable $11.70 \mathrm{~dB}$, the performance over the range where the system's response is the greatest, $90-220 \mathrm{~Hz}$, was much 

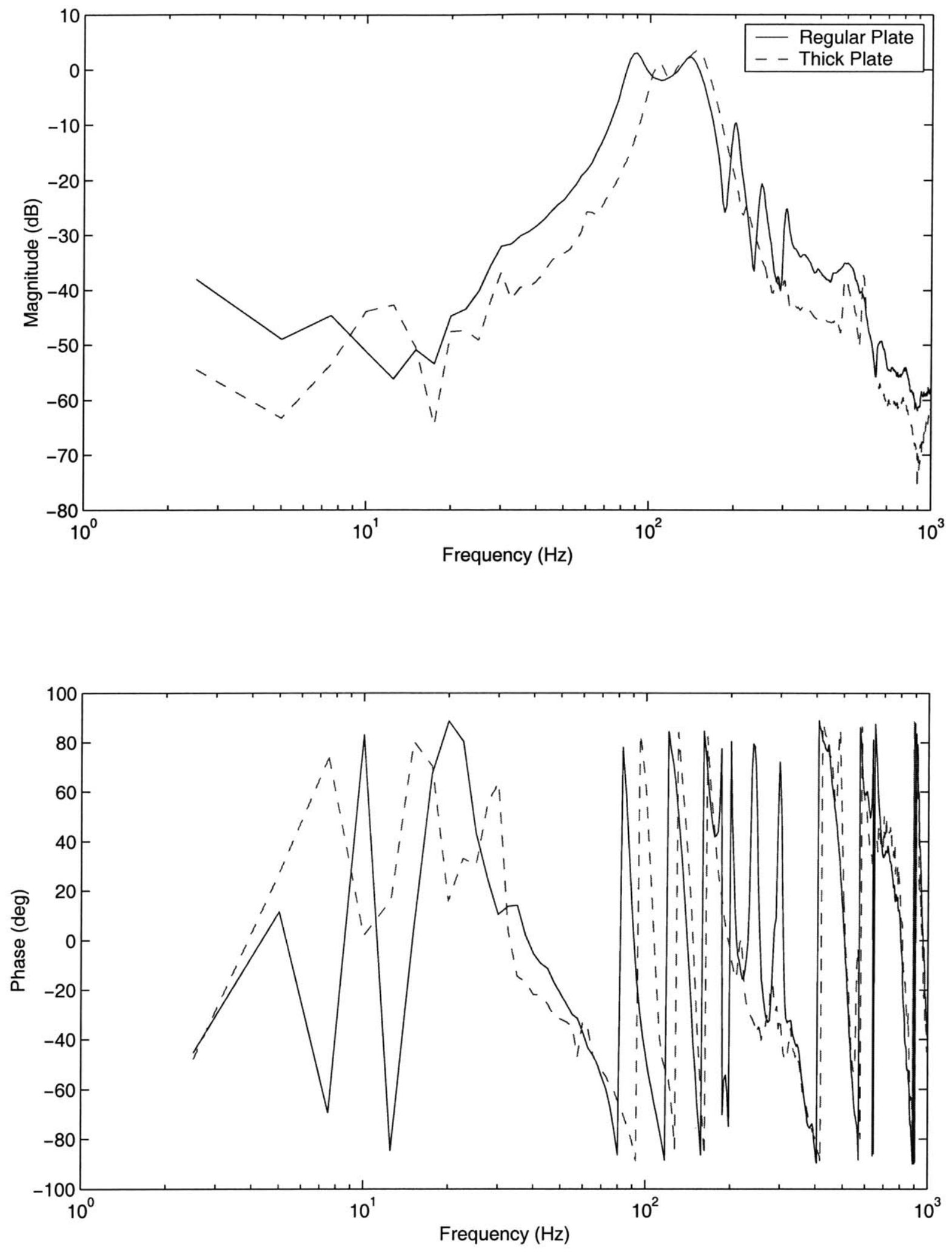

Figure 5-1: Regular Plate vs. Thick Plate (twice the thickness) 

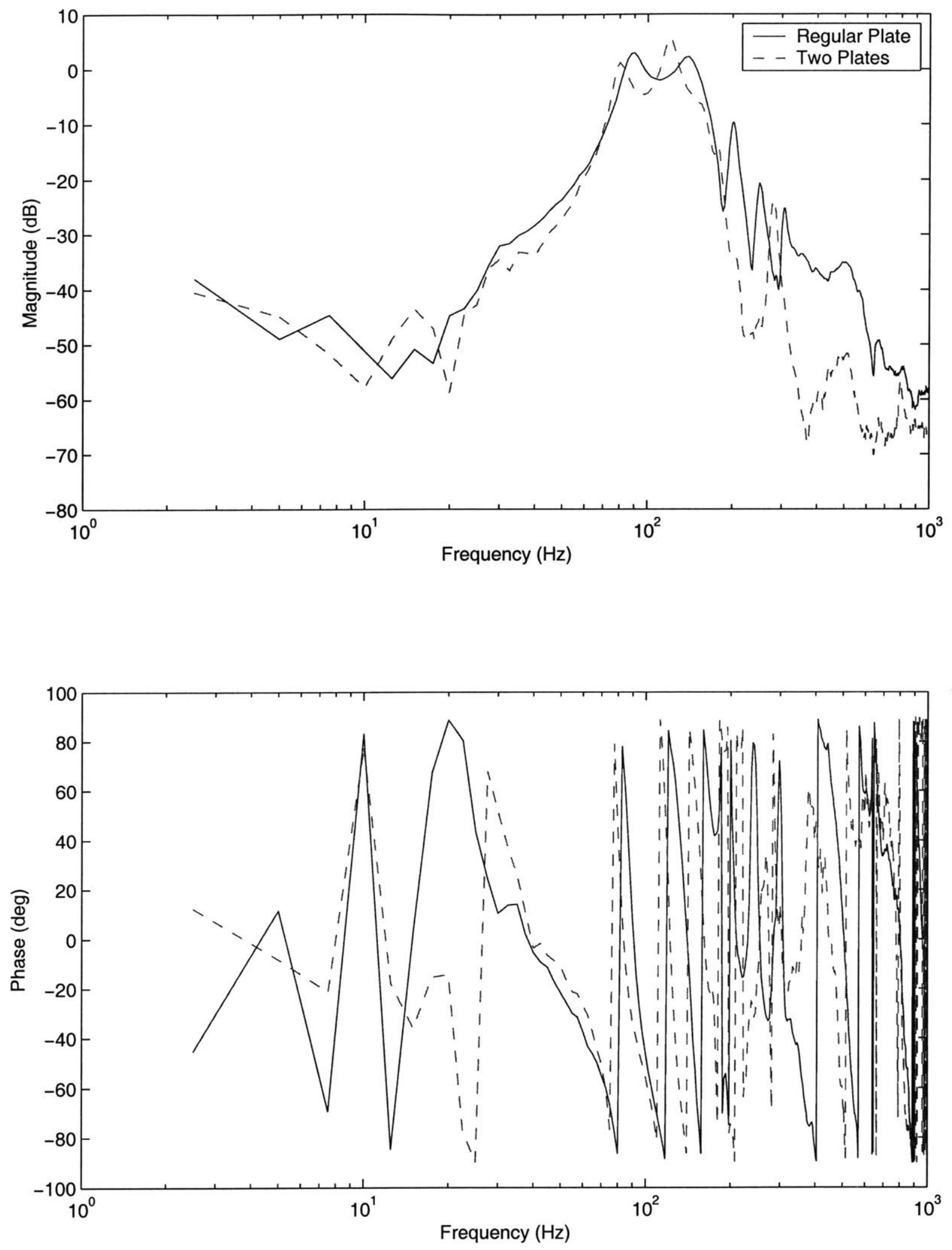

Figure 5-2: Regular Plate vs. Two Plates (9.875 in apart) 
better. The two plate system showed a reduction of $1.70 \mathrm{~dB}$ while the thicker plate showed an increase of $0.98 \mathrm{~dB}$ over the range of 90-220 Hz. Overall, the two plate system performed significantly better, posting a broadband reduction of $1.92 \mathrm{~dB}$ from 40 to $1000 \mathrm{~Hz}$.

\subsection{Active Control}

\subsubsection{MIMO Controller}

The test chamber configuration used to test the Multi-Input Multi-Output (MIMO) Controller was the Dual Layer Chamber Configuration shown in Figure 2-3. The control loop diagram for the MIMO Controller is shown in Figure 5-3. The goal of the compensator was to minimize the broadband response from the disturbance speaker, which represents the volume outside the fairing, to Microphone 1, which represents the volume inside the fairing.

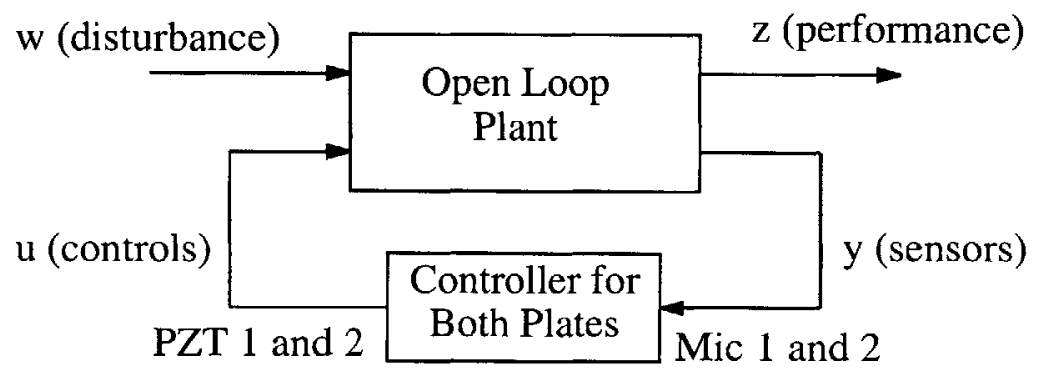

Figure 5-3: MIMO Controller Configuration

\section{Open Loop}

The components of the test chamber used for this configuration can be separated into three categories: sensors, actuators and the disturbance source. The feedback sensors $(\boldsymbol{y})$ were Microphones 1 and 2 (referred to as Mic1, Mic2), the actuators ( $\boldsymbol{u}$ ) were PZT1 and PZT2 and the disturbance source $(\boldsymbol{w})$ was the bottom disturbance speaker. The feedback sensors were also the performance sensors $(\boldsymbol{z})$. Microphone 4 (Mic4) was used to provide measurements of the sound field at the disturbance 
speaker, so that transfer functions could be determined from the disturbance speaker to the performance sensors.

Because both plates were actuated upon in the Dual Layer Chamber Configuration for the MIMO Controller, the $G_{y u}$ block was a $2 \times 2$ transfer function matrix. The four $G_{y u}$ transfer functions were PZT1-to-Mic1, PZT1-to-Mic2, PZT2-to-Mic1 and PZT2-to-Mic2. The $G_{y w}$ block was a $2 \times 1$ transfer function matrix that included the transfer functions Spkr-to-Mic2 and Spkr-to-Mic1. The $G_{y u}$ transfer functions are shown in Figure 5-4, and the $G_{y w}$ transfer functions are shown in Figure 5-5. In this configuration, $G_{z w}$ is identical to $G_{w u}$ and $G_{z u}=0$.

The modes of the system are easily determined by looking at the $G_{y w}$ transfer functions. The first and second plate symmetric modes of the plate are at $77.9 \mathrm{~Hz}$ and $275.6 \mathrm{~Hz}$ respectively, and the first and second chamber acoustic modes are at $118.8 \mathrm{~Hz}$ and $256.4 \mathrm{~Hz}$, respectively. The symmetric plate modes are highly observable and controllable. In contrast, the asymmetric modes of the plate, the acoustic modes of the lower chamber and the dynamic modes of the speaker are much less observable and controllable, and they appear in the transfer functions as near polezero cancellations. It is also interesting to note the difference between the frequencies of the acoustic modes determined experimentally and the frequencies of the acoustic modes determined theoretically, which are about $168 \mathrm{~Hz}$ and $336 \mathrm{~Hz}$, respectively. The reason for the discrepancy between the theoretical and experimental values can be attributed to the coupling of acoustics with the dynamics of the speaker. The frequency of the fundamental acoustic mode is pushed down from $168 \mathrm{~Hz}$ to $118.8 \mathrm{~Hz}$ and the speaker mode is pushed up from $171 \mathrm{~Hz}$ to $178 \mathrm{~Hz}$. In fact, in this configuration, with the inter-plate spacing at $9.875 \mathrm{in}$, the speaker-acoustic coupling is so strong that the first acoustic mode dominates all other modes. This was not the case when the inter-plate spacing was smaller, and the first structural mode had the greatest magnitude. 

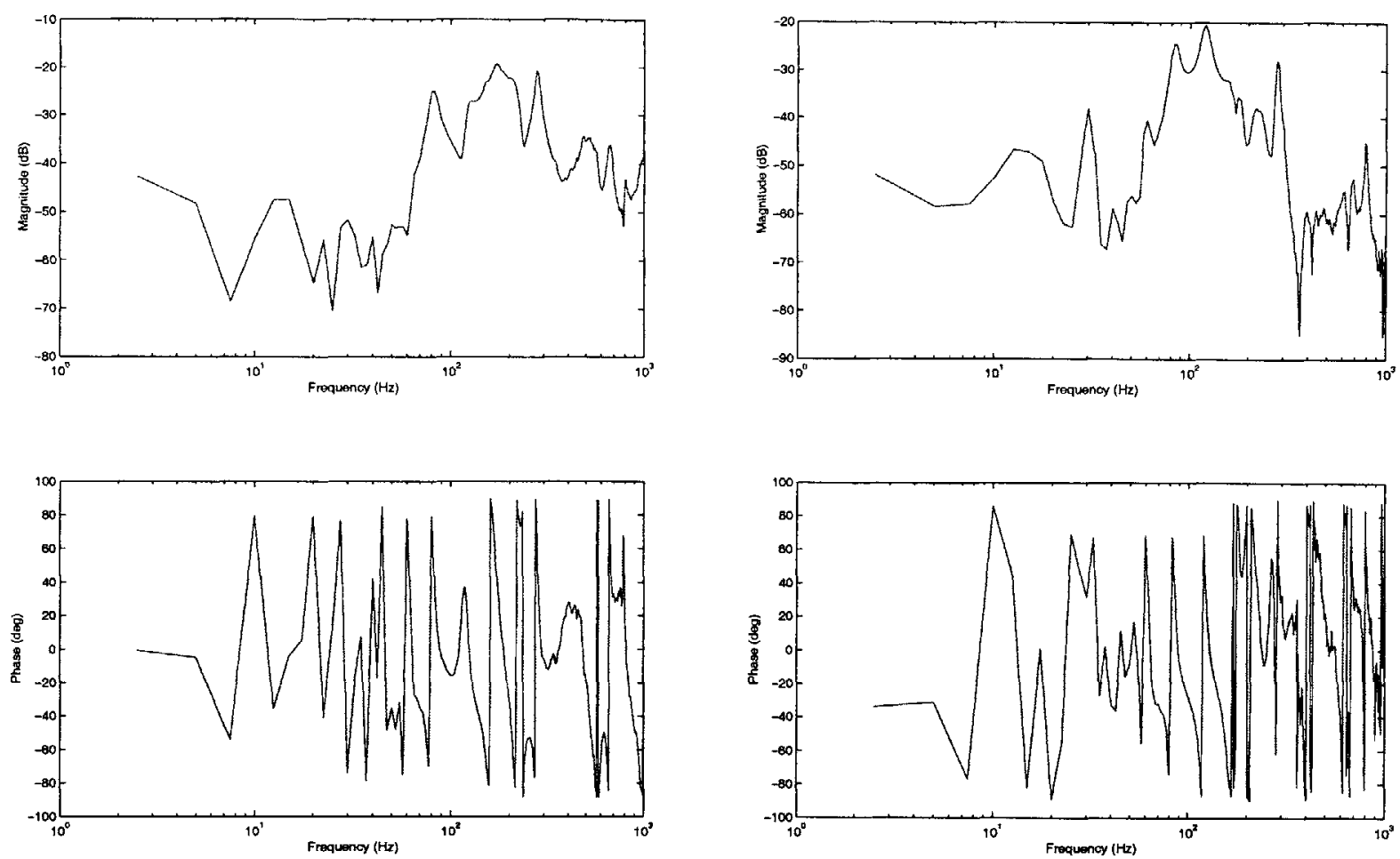

$G_{y_{1} u_{1}}:$ PZT1-to-Mic1

$G_{y_{1} u_{2}}:$ PZT2-to-Mic1
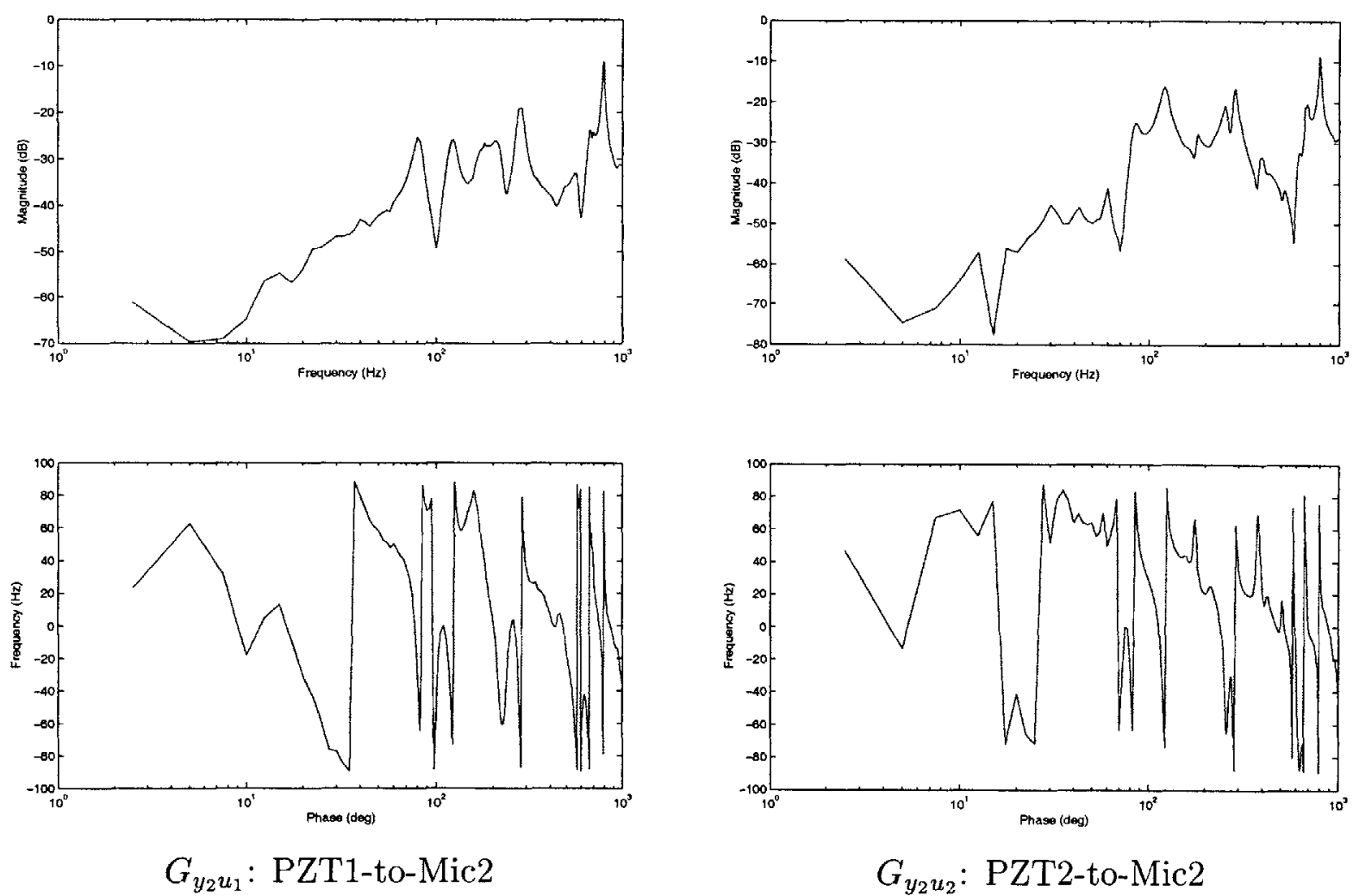

Figure 5-4: MIMO Controller Open Loop $G_{y u}$ Transfer Functions 

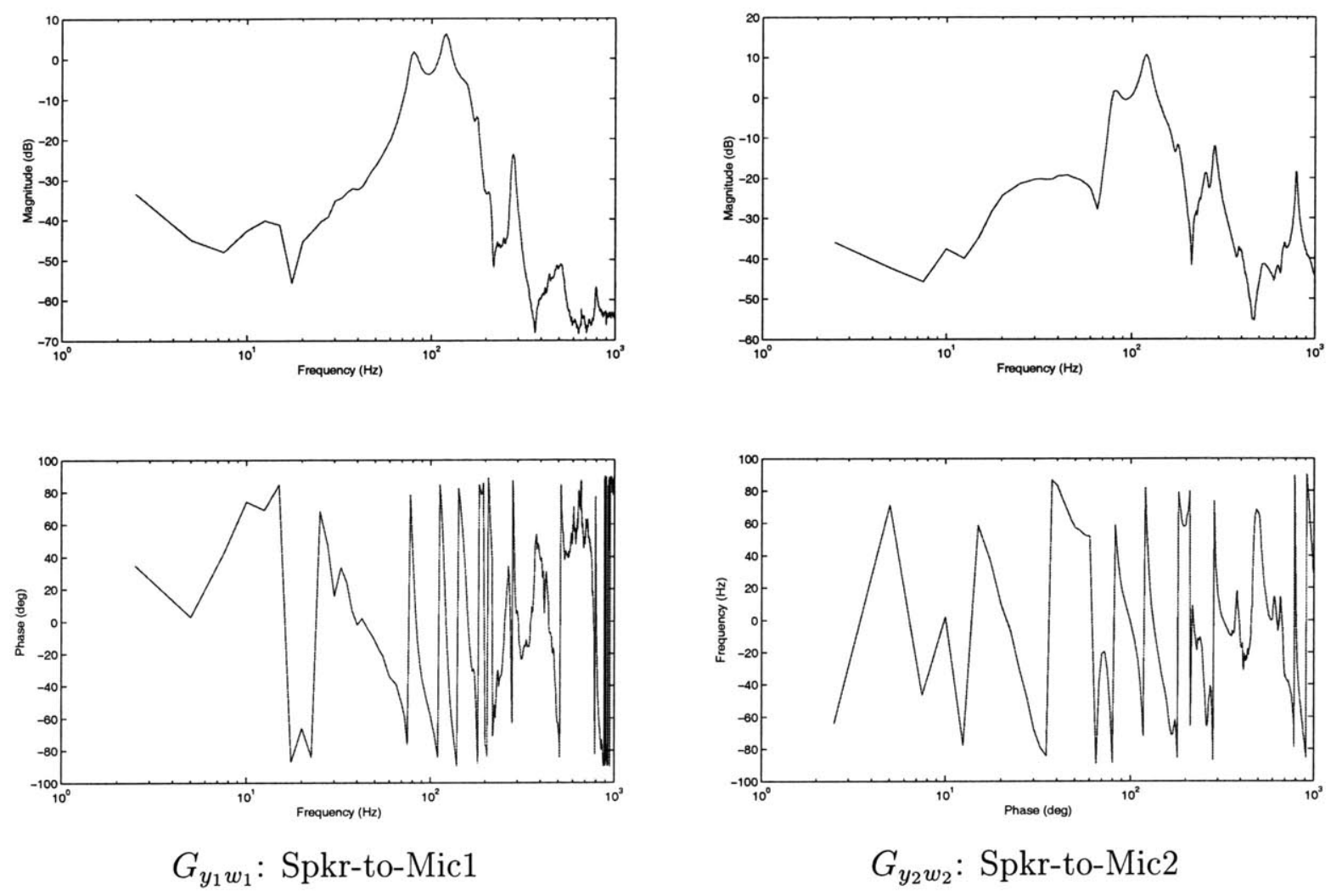

Figure 5-5: MIMO Controller Open Loop $G_{y w}$ Transfer Functions 


\section{Controller Design}

The compensator used for transmission control was designed using the LQG method described in Chapter 4 and was implemented using a dSPACE digital computer operating at a sampling frequency of $5 \mathrm{kHz}$. The size of the MIMO Controller was 75 states, which was near the limit of the digital computer's processing capabilities.

Figure 5-6 shows the compensator transfer functions. The controller responses are large at the frequencies where the corresponding states have been penalized by entering positive values in the $Q$ matrix in the LQR cost function. The three peaks in the $G_{u y}$ transfer functions correspond to penalizing the modes at $77.9,118.8$ and $275.6 \mathrm{~Hz}$.

Figure 5-7 shows the Nichols plot that is derived from the loop transfer function formed by interconnecting the plant $G_{y u}$ and the compensator. The Nichols plot for the top and bottom plate systems have the same outline because they share the same dynamics and have the same control and performance penalties on the same modes. The gain and phase margins of the system are about $37 \mathrm{~dB}$ and $150^{\circ}$.

\section{Closed Loop Performance}

The open loop and closed loop transfer functions for the MIMO Controller are plotted in Figure 5-8. The structural-acoustic system was most sensitive to disturbances at $118.8 \mathrm{~Hz}$, where the magnitude of the transfer functions are the greatest. Because the magnitude of this acoustic mode was greater than any other, reducing the system response at this frequency would improve broadband performance the most. However, there is a limit to how much reduction can be attained. Bode's gain-phase integral relation and sensitivity integral posit that poles and zeros in the right half plane adversely affect the performance of the compensated system [17]. The water bed effect of the system's sensitivity function ensures that the sensitivity function must have regions that are greater than unity if there are regions that are less than unity, which implies that a system's response must increase at some frequency region if it is reduced at some other frequency region. Although it would be preferable to minimize 

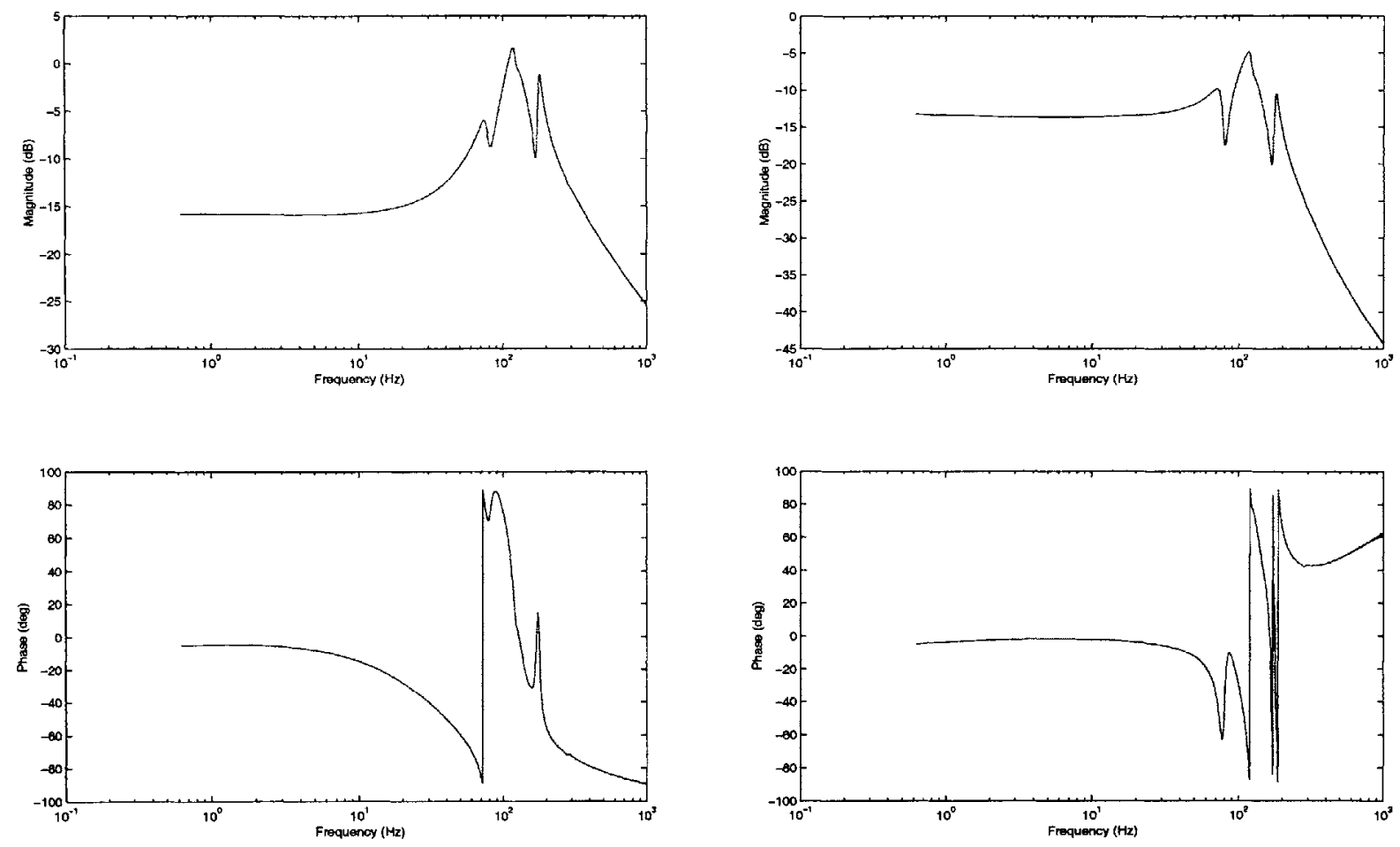

$G_{u_{1} y_{1}}:$ Mic1-to-PZT1

$G_{u_{1} y_{2}}:$ Mic2-to-PZT1
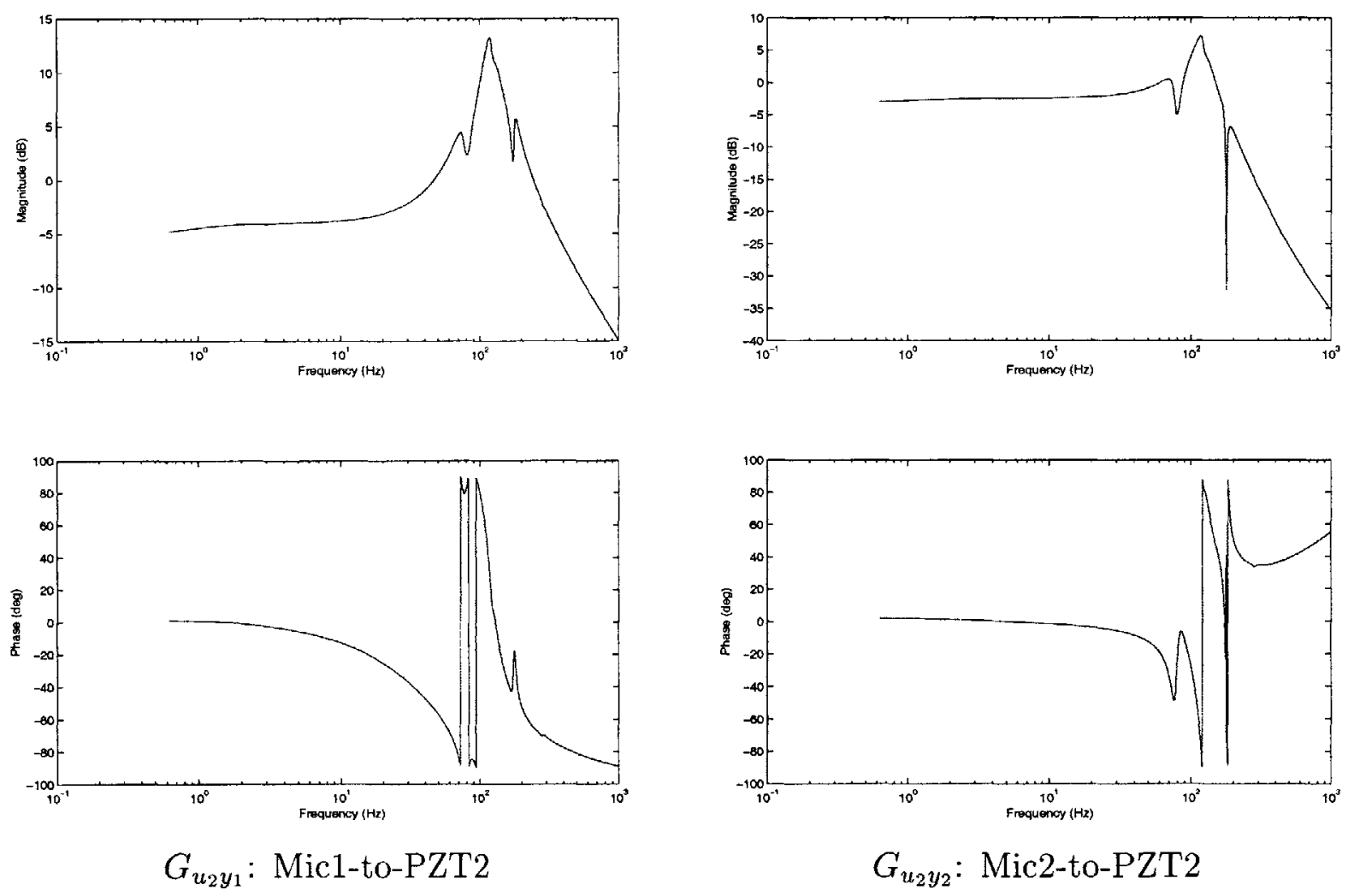

Figure 5-6: MIMO Controller $G_{u y}$ Transfer Functions 

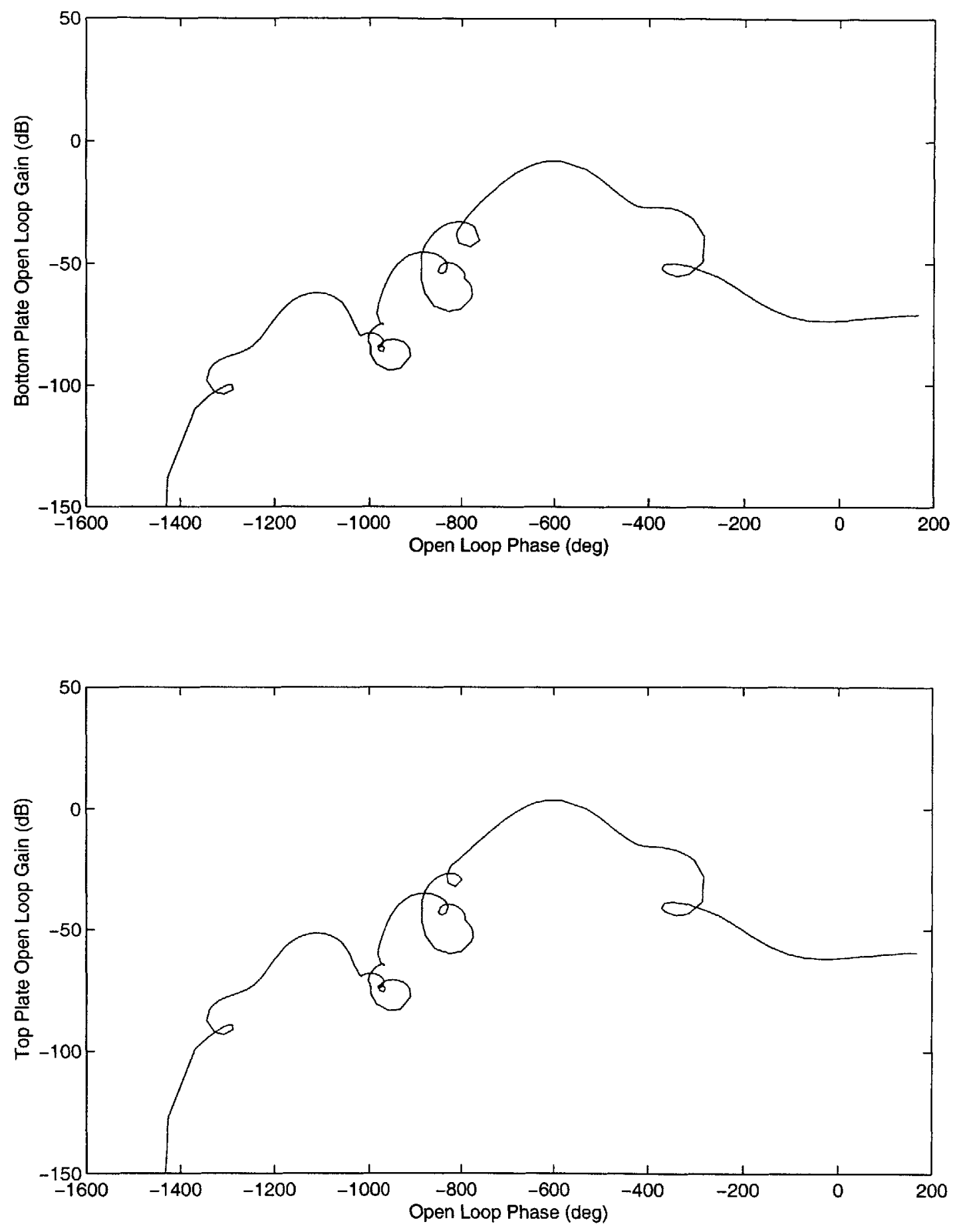

Figure 5-7: MIMO Controller Nichols Chart 

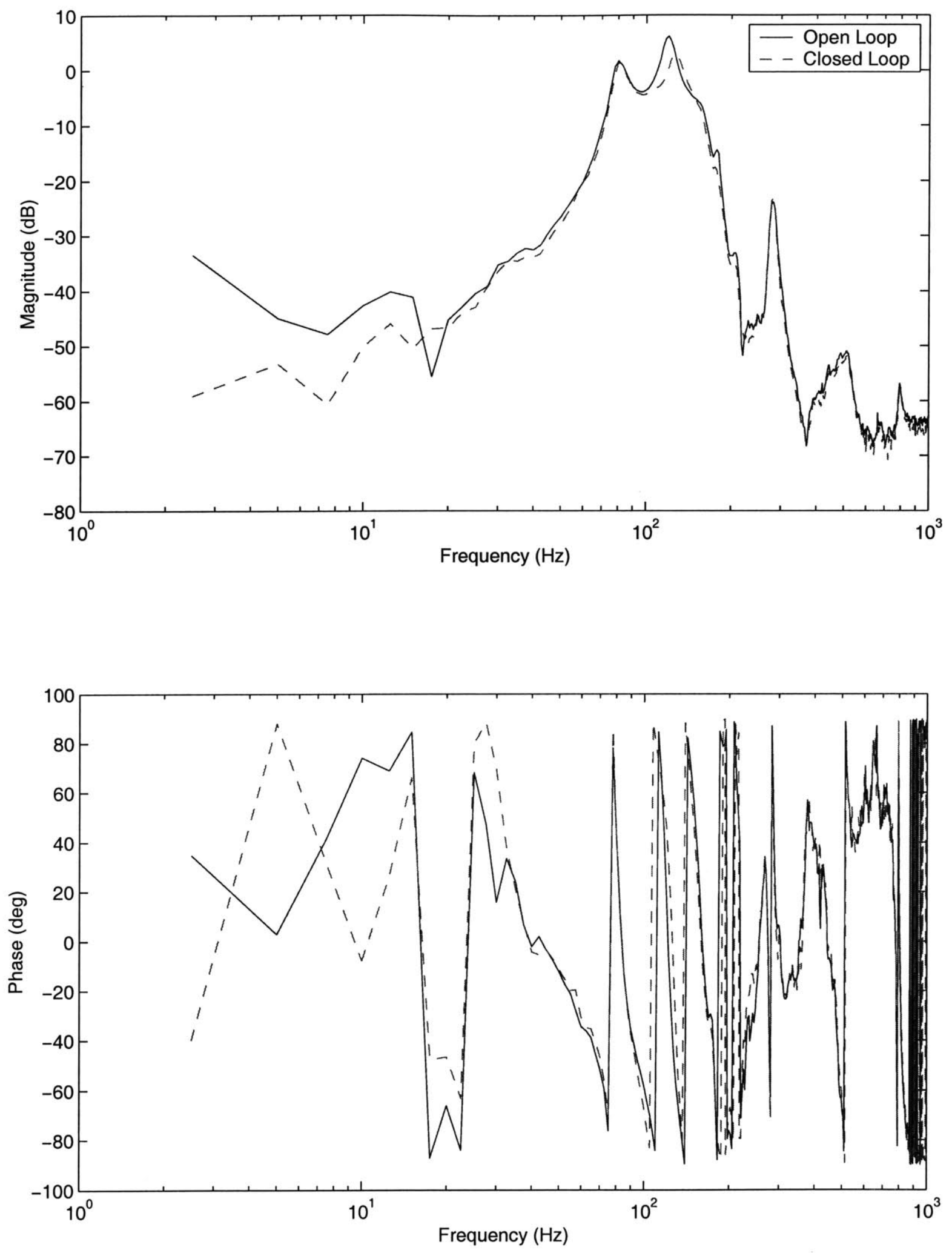

Figure 5-8: MIMO Controller Performance 
the system response over the entire broadband region, this goal may not be realized; the response may "spike" in a frequency range where the designer wants the response to be minimized.

The broadband performance for the MIMO Controller from $40-1000 \mathrm{~Hz}$ was a $1.33 \mathrm{~dB}$ reduction from the speaker to Mic1. The lower frequency threshold was set at $40 \mathrm{~Hz}$ and not at 1 or $10 \mathrm{~Hz}$ because the coherence function over the 1-40 $\mathrm{Hz}$ range was low. Therefore, the results over that range were not consistent and the data was not reliable. However, the frequency response of the system from $40 \mathrm{~Hz}$ onward had high coherence and is suitable to be used as a broadband performance metric.

The frequency range that contributed most to the broadband reduction was at the first acoustic mode $(118.8 \mathrm{~Hz})$ where the response was reduced by $6.27 \mathrm{~dB}$. The first plate mode and the second acoustic mode were reduced by $1.0514 \mathrm{~dB}$ and $3.79 \mathrm{~dB}$, respectively. The closed loop transfer function plotted in Figure 5-8 provided the best broadband performance among all the MIMO Controllers tested. When the gain was increased further, the first acoustic mode at $118.8 \mathrm{~Hz}$ was attenuated more. However, the energy only shifted to a slightly higher frequency at about $130 \mathrm{~Hz}$, and broadband performance suffers as the amount of energy being transmitted near $130 \mathrm{~Hz}$ overwhelms the amount of energy being reduced at $118.8 \mathrm{~Hz}$. The results from using the MIMO Controller are summarized in Table 5.1.

Table 5.1: MIMO Controller Performance Summary

\begin{tabular}{l|l|l||r|r}
\hline Mode & \multirow{2}{*}{$\begin{array}{l}\text { Frequency } \\
\end{array}$} & Reduction & \multicolumn{2}{|c}{ Reduction (dB) } \\
\cline { 4 - 5 } & $(\mathrm{Hz})$ & Range (Hz) & SpkrtoMic1 (Top) & SprktoMic2 (Bot) \\
\hline \hline $1^{\text {st }}$ Structural & 77.9 & $75-80$ & 1.05 & 0.97 \\
$1^{\text {st }}$ Acoustic & 118.8 & $116-120$ & 6.27 & 6.50 \\
$1^{\text {st }}$ Acoustic & 130.0 & $125-135$ & -1.19 & -0.92 \\
$2^{\text {nd }}$ Acoustic & 177.5 & $175-180$ & 3.79 & 1.38 \\
\hline \multicolumn{2}{l}{ Broadband $(40-1000 \mathrm{~Hz})$} & 1.33 & 1.39 \\
\hline
\end{tabular}

\footnotetext{
${ }^{\dagger}$ The MIMO Controller changes the acoustic characteristics of the system, and the peak of the $1^{\text {st }}$ acoustic mode shifts from the open loop frequency of $118.8 \mathrm{~Hz}$ to the closed looped frequency of $130.0 \mathrm{~Hz}$
} 


\subsubsection{Successive Loop Closure Controller}

The test chamber configuration used to test the Successive Loop Closure (SLC) Controller was the Dual Layer Chamber Configuration shown in Figure 2-3. The control loop diagram for the SLC Controller is shown in Figure 5-9. The inner control loop, which had the bottom plate controller, was designed first, and the resulting compensated systems served as the open loop plant for the outer control loop, which was closed using the top plate controller. The goal of the overall compensator was to minimize the broadband response from the disturbance speaker, which represents the volume outside the fairing, to Microphone 1, which represents the volume inside the fairing.

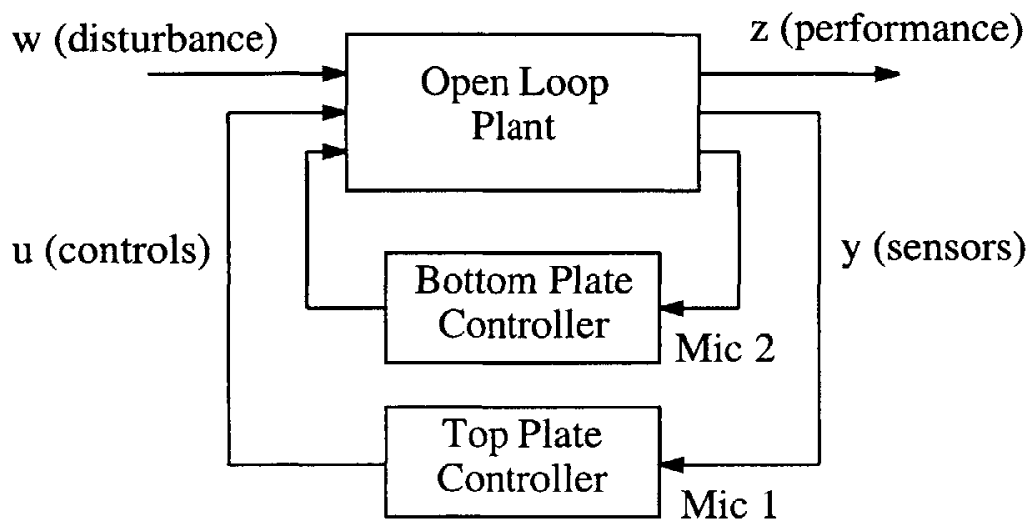

Figure 5-9: Successive Loop Controller Configuration

\section{Open Loop}

The components of the test chamber used for this configuration are the same as those described for the MIMO Controller described in Section 5.2.1. Mic1 and Mic2 were the feedback and the performance sensors $(\boldsymbol{y}$ and $\boldsymbol{z})$. PZT1 and PZT2 were the actuators $(\boldsymbol{u})$, and the bottom speaker was the disturbance source $(\boldsymbol{w})$.

Whereas the MIMO Controller had two inputs and two outputs, the SLC Controller has two controllers with one input and one output each. The $G_{y u}$ and the $G_{y w}$ blocks were $1 \times 1$ for each controller. The size of $G_{y u}$ block was smaller than that of the MIMO Controller because the cross-coupling transfer functions (PZT1- 
to-Mic2 and PZT2-to-Mic1) were not included in the plant description. Each of the two plates had one sensor microphone near it, but the sensor microphone could not differentiate whether the acoustic field it was measuring was due to one plate or to the other. In addition, the compensator for each plate could not estimate what fraction of the response was due to the other plate's actuation because it did not have access the other plate's control input, $\boldsymbol{u}$. Therefore, the cross-coupling transfer functions did not serve any purpose and were not included in the plant descriptions. The $G_{y u}$ and $G_{y w}$ transfer functions for the bottom plate compensator and for the top plate compensator are shown in Figures 5-10 and 5-11, respectively.

Mic2-to-Mic1 was used instead of Spkr-to-Mic1 for the top plate controller $\left(G_{y w}\right)$ because the acoustic cavity pressure acts as the originating noise source for Plate 1. Therefore, Plate 2, actively controlled or not, acts as a shaping filter for the disturbance source. Intuitively, Mic2-to-Mic1 is a better choice than Spkr-to-Mic1 because Mic2-to-Mic1 describes how much energy is flowing through Plate 1, which is the quantity that is to be minimized for active structural control. Although Spkr-toMicl was not used in the plant description, it is the performance metric $(z)$ that describes how well the closed loop dual layer system is working compared to its open loop counterpart because it represents how much acoustic energy is flowing across both plates or the entire active structural system. The two performance metrics, Spkr-to-Mic1 and Spkr-to-Mic2, are shown in Figure 5-12.

The modes of the system are determined by examining the $G_{y w}$ transfer functions. It is interesting to note that the natural frequencies for the top and bottom plate systems were different. The structural modes of the bottom plate system were at $80.4 \mathrm{~Hz}$ and at $280.7 \mathrm{~Hz}$, and the acoustic modes for the bottom plate system were at $118.3 \mathrm{~Hz}$ and $260.0 \mathrm{~Hz}$. In comparison, the top plate system structural modes were at $63.3 \mathrm{~Hz}$ and at $262.4 \mathrm{~Hz}$, and its acoustic modes were at $142.5 \mathrm{~Hz}$ and $201.3 \mathrm{~Hz}$. As in the case of the MIMO Controller, the speaker dynamics interacted with the chamber acoustics, pushing down the first acoustic mode frequencies down from a theoretical value of $168 \mathrm{~Hz}$ and pushing up the the speaker mode frequency from $171 \mathrm{~Hz}$. 

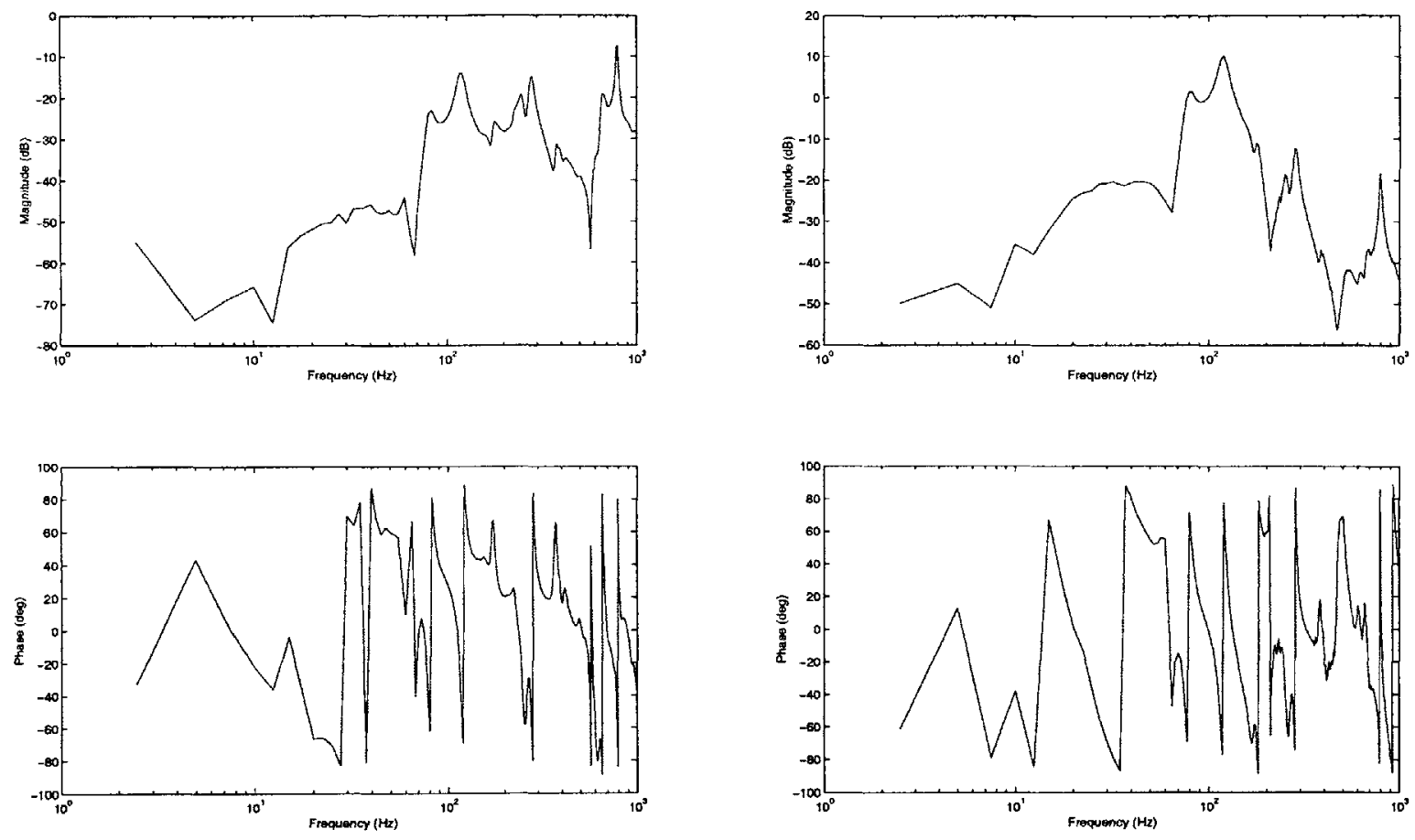

$G_{y u}:$ PZT2-to-Mic2

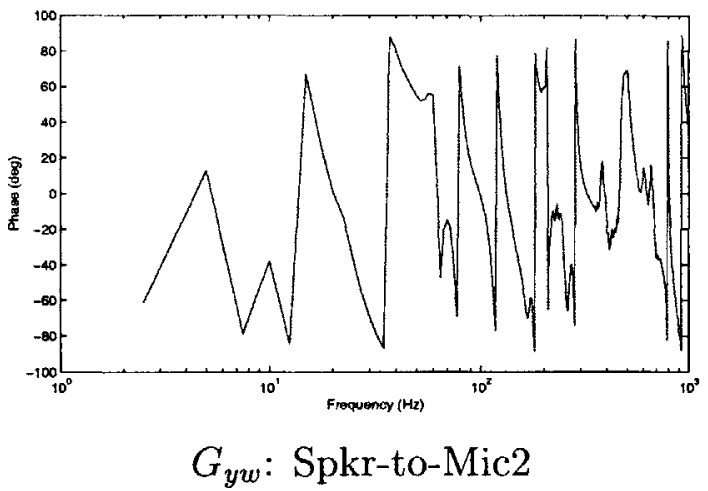

Figure 5-10: SLC Controller Bottom Plate Open Loop $G_{y u}$ and $G_{y w}$ Transfer Functions
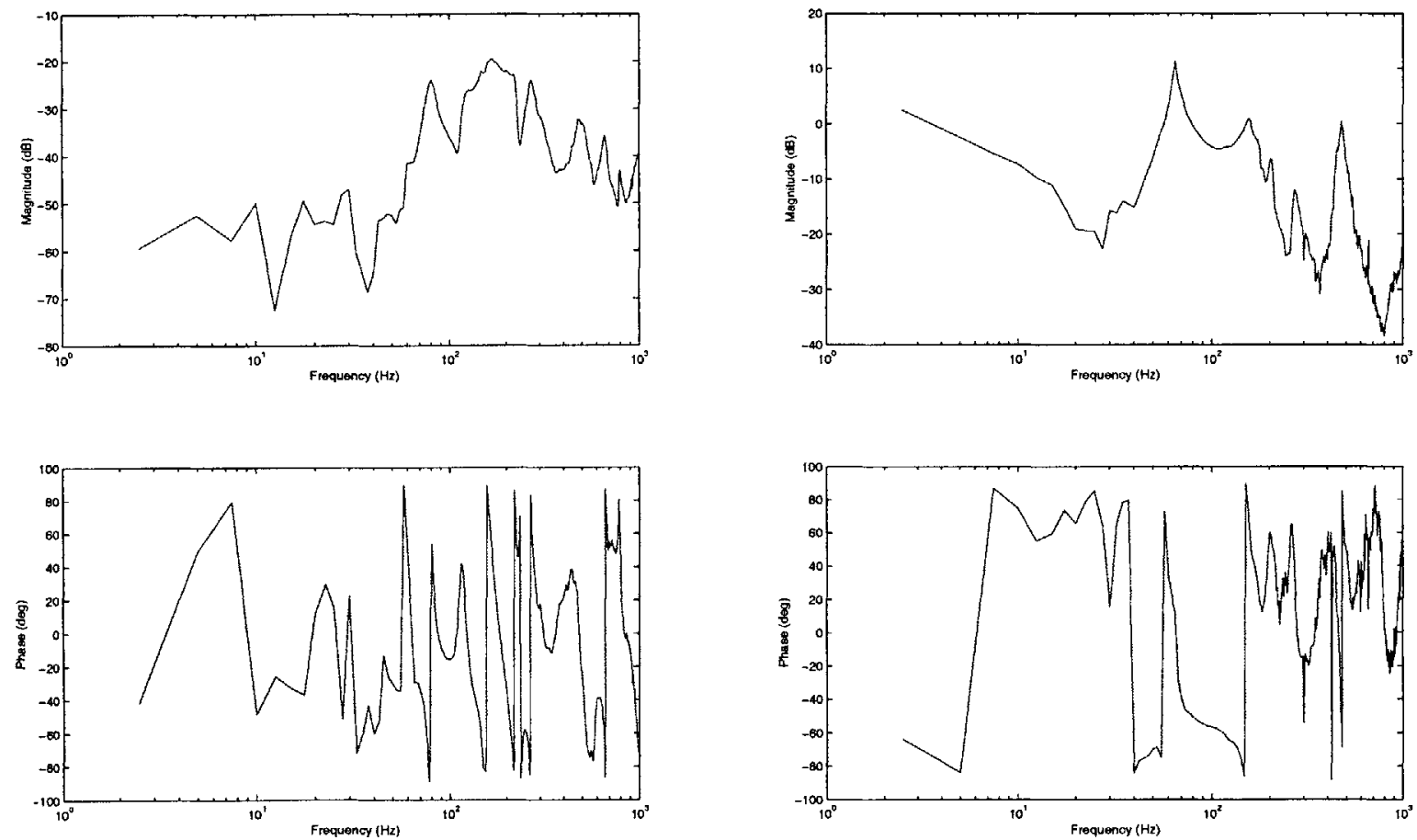

$G_{y u}:$ PZT1-to-Mic1

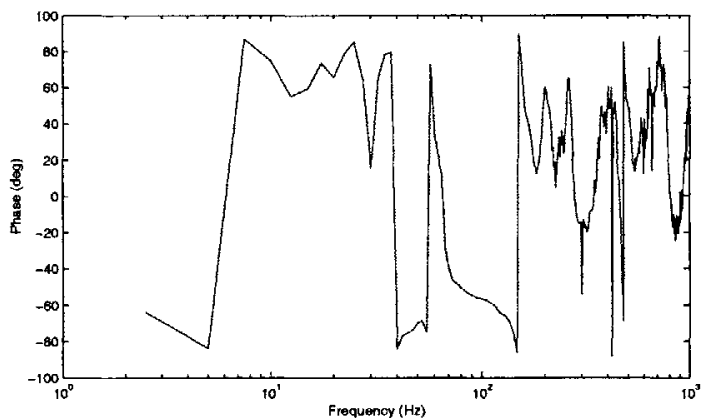

$G_{y w}:$ Mic2-to-Mic1

Figure 5-11: SLC Controller Top Plate Open Loop $G_{y u}$ and $G_{y w}$ Transfer Functions 

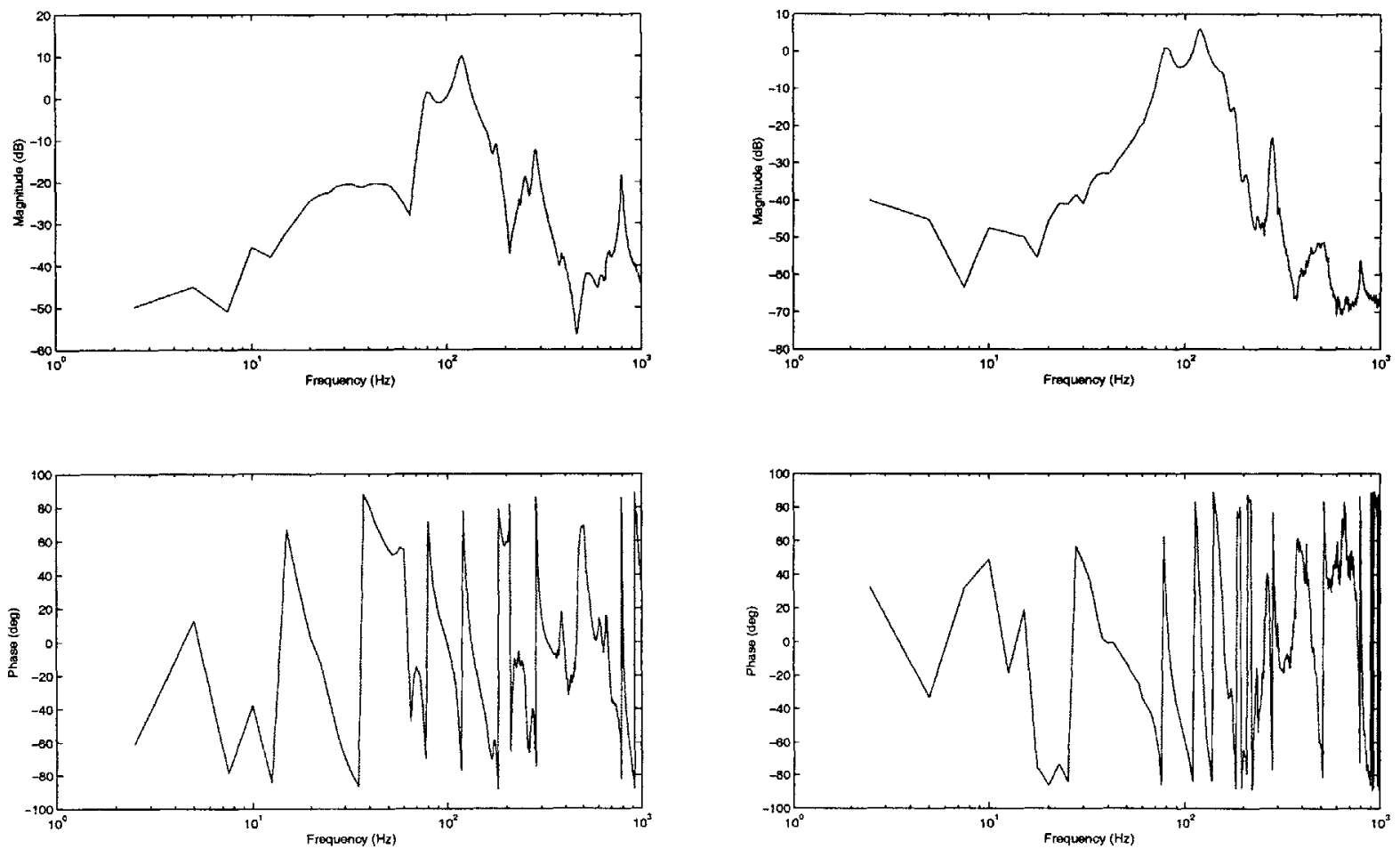

Bottom Plate $G_{z w}:$ Spkr-to-Mic2

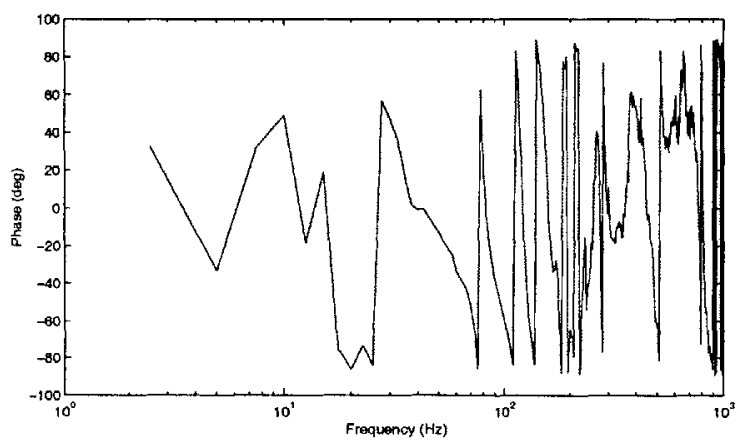

Top Plate $G_{z w}:$ Spkr-to-Mic1

Figure 5-12: SLC Controller Open Loop $G_{z w}$ Transfer Functions for Both Plates 


\section{Controller Design}

The compensator used for transmission control was designed using the LQG Method described in Chapter 4 and was implemented using a dSPACE digital computer operating at a sampling frequency of $5 \mathrm{kHz}$. The sizes of the bottom plate and top plate controllers were fifty-two and fifty-four states, respectively, which was near the limit of the digital computer's processing capabilities.

Figure 5-13 shows the compensator transfer functions. The controller responses are large at the frequencies where the corresponding states have been penalized by entering positive values in the $Q$ matrix in the LQR cost function. The bottom plate controller penalizes the modes at 80.4, 118.3, 123.5, 173.9, 208.8, 235.6, 260.0, 280.7, 376.1 and $794.7 \mathrm{~Hz}$. The top plate controller penalizes the modes at 63.3, 79.4, 158.6, $171.3,269.0$ and $538.2 \mathrm{~Hz}$. The peaks of $G_{u y}$ in Figure 5-13 correspond to these targeted modes, although many of them are overwhelmed by those which have the greatest state penalties.

Figure 5-14 shows the Nichols plot that is derived from the loop transfer function formed by interconnecting the plant $G_{y u}$ and the compensator. The bottom and top plate systems have gain and phase margins of $30 \mathrm{~dB}$ and $180^{\circ}$, and $4 \mathrm{~dB}$ and $110^{\circ}$, respectively.

\section{Closed Loop Performance}

The results obtained from implementing the Successive Loop Closure Controller was better than the results from any of the other tested controller configurations. One of the main differences between the SLC Controller and the MIMO Controller is how the controllers are designed. Although the open loop transfer functions for the Spkr-toMic2 was very similar to that used to design the MIMO Controller, the other "open loop" transfer function, Mic2-to-Mic1, for the top plate controller was significantly different from the one used for the MIMO controller because the SLC Controller's Mic2-to-Mic1 transfer function was taken while the bottom plate system was being actively controlled. In addition, the fact that there was a separate controller for each 

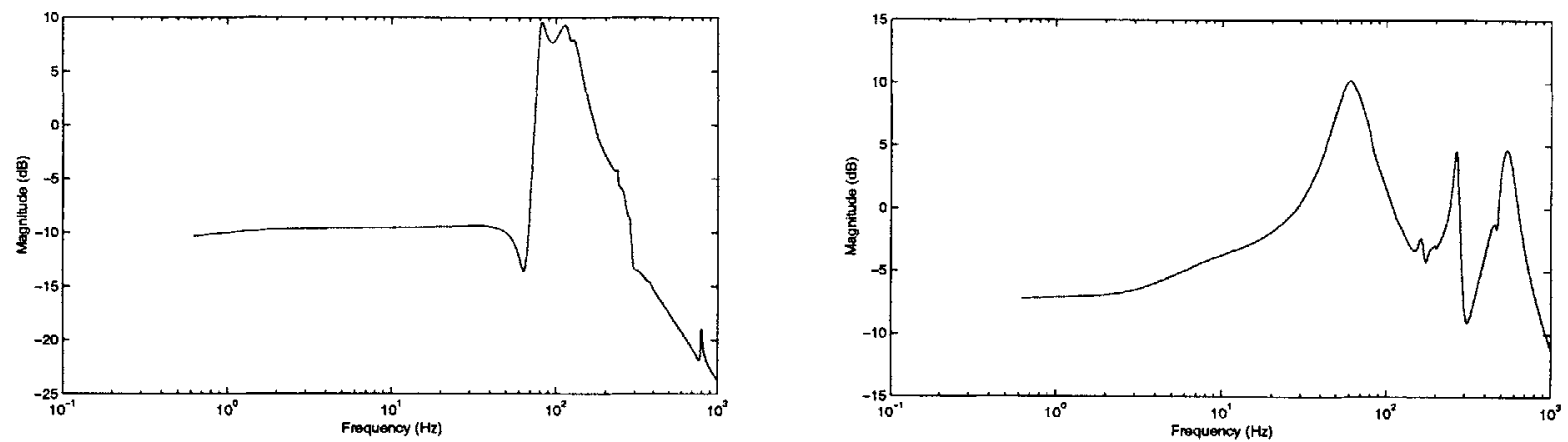

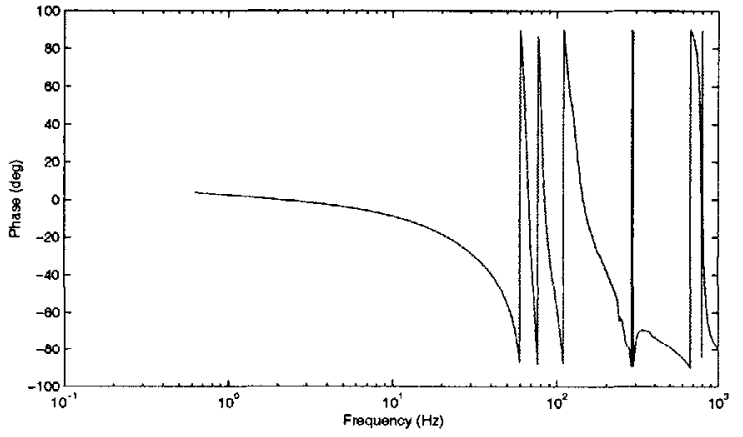

Bottom Plate $G_{u_{2} y_{2}}$ : Mic2-to-PZT2

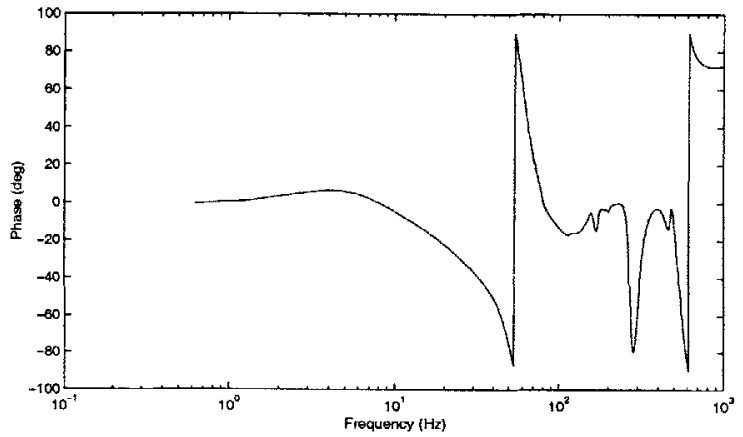

Top Plate $G_{u_{1} y_{1}}:$ Mic1-to-PZT1

Figure 5-13: SLC Controller $G_{u y}$ Transfer Functions 

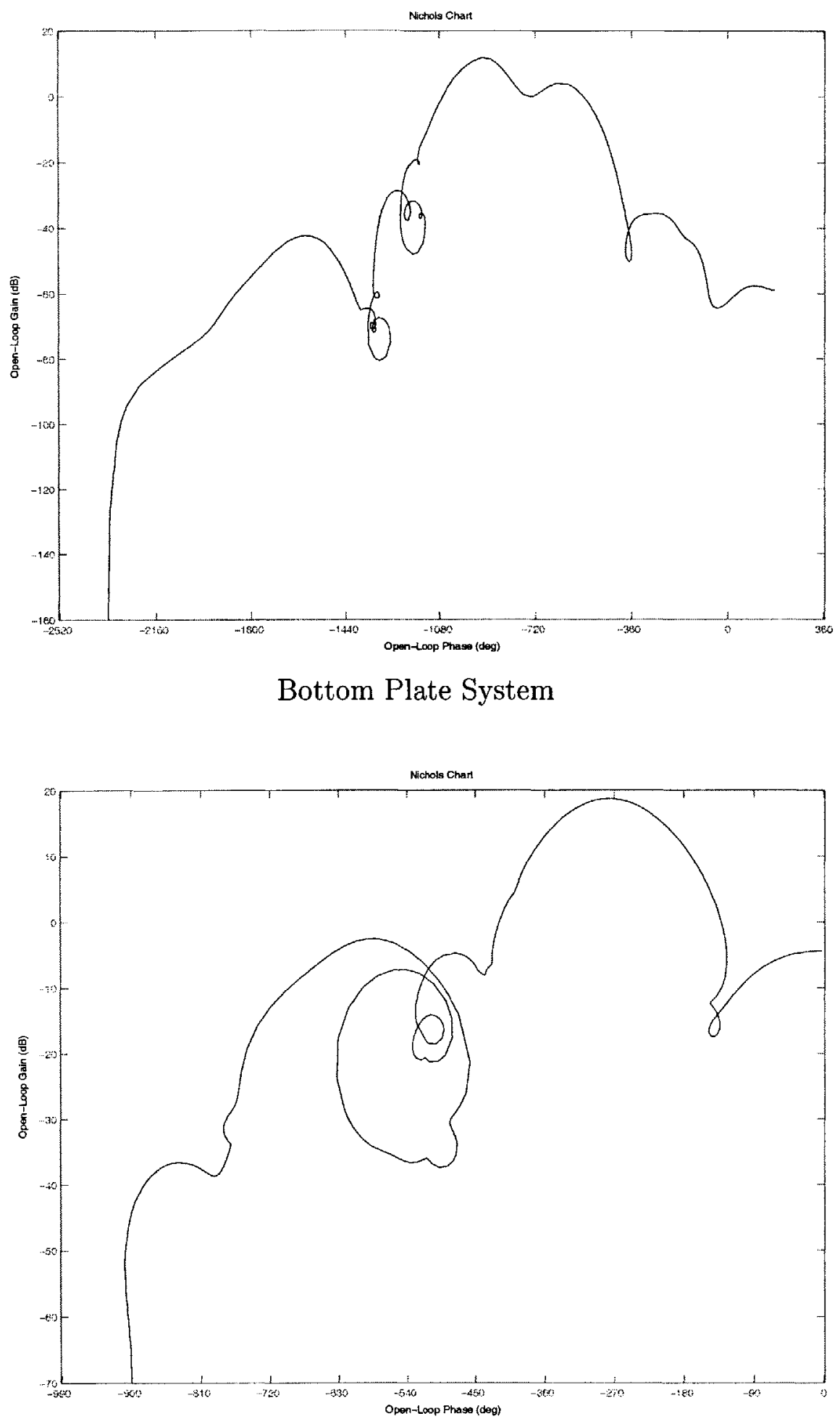

Top Plate System

Figure 5-14: SLC Controller Nichols Chart 
plate significantly increased the flexibility that the designer had in targeting modes. If a mode was nullified by the lower plate controller, then the top plate could target a more prominent mode instead of the same mode, and therefore, improve broadband performance. However, this flexibility in the targeting of modes comes at a price; the SLC Controller takes longer to design and to implement, and there is no easy way to predict the how the control gain of the top plate system should compare to the control gain of the lower plate system. These "optimal" gain values have to be determined empirically through experimentation.

Figures 5-15 and 5-16 show the open and closed loop transfer functions of the SLC Controller. Figure 5-15 shows the system performance of Spkr-to-Mic1 when only the bottom plate is active, and Figure 5-16 shows the same performance metric when both plates are active. On its own, the bottom plate controller was very effective in reducing the first structural and acoustic modes such that the closed loop transfer function was relatively flat from $70-130 \mathrm{~Hz}$. The first structural mode was reduced by $1.70 \mathrm{~dB}$, and the first acoustic mode was reduced by $6.75 \mathrm{~dB}$. The fact that the first acoustic mode had the greatest magnitude and that it experienced the greatest reduction made it the single largest contributor toward improving broadband performance. The system also proved to be quite stable over a large range of gain as its large stability and phase margins had indicated. A summary of the SLC Controller using only the bottom plate controller can be found in Table 5.2 .

Although the performance when using only the bottom plate controller was good, closing the loop by introducing the top plate controller improved performance significantly. With both of the controllers of the SLC Controller active, the system's frequency response from $70-130 \mathrm{~Hz}$ decreased by about $2 \mathrm{~dB}$. The attenuation of the first structural and the first acoustic modes were increased to $6.42 \mathrm{~dB}$ and $9.61 \mathrm{~dB}$, respectively. The top plate controller was also able to target the second structural mode and was able to reduce its response at $280.7 \mathrm{~Hz}$ by $7.25 \mathrm{~dB}$. However, at the gains necessary to attain theses reductions in modal responses, the top plate controller started to exhibit instabilities, especially at the second acoustic frequency at $173.9 \mathrm{~Hz}$. The spike at the second acoustic mode was an increase of $9.51 \mathrm{~dB}$. The 
closed loop response shown in Figure 5-16 represents the best performance that was attainable. Increasing the gain would drive the system unstable, and reducing the gain would decrease the second acoustic mode response but would also begin to eliminate the reductions attained at the first and second structural modes and at the first acoustic mode. The Nichols chart in Figure 5-14 corroborates the experimental results as the top plate system's gain margin was only about $4 \mathrm{~dB}$ as compared to the $30 \mathrm{~dB}$ for the bottom plate system's. The problem was exacerbated by the fact that the pole associated with the second acoustic mode is closely coupled with the disturbance speaker dynamics and has very poor controllability and observability characteristics. Even so, attempts were made to try to remove the destabilizing effects by redesigning the controllers, but the problem persisted. However, even with the increase in the system's response at the second acoustic frequency, the broadband characteristics of the SLC Controller improved to $2.08 \mathrm{~dB}$, which was better than any other controller configuration tested. Table 5.3 summarizes the results obtained when controlling both plates.

Table 5.2: SLC Controller (Bottom Only) Performance Summary

\begin{tabular}{l|l|l||r|r}
\hline Mode & \multirow{2}{*}{$\begin{array}{l}\text { Frequency } \\
\text { (Hz) }\end{array}$} & $\begin{array}{l}\text { Reduction } \\
\text { Range (Hz) }\end{array}$ & \multicolumn{2}{|c}{ Reduction (dB) } \\
\cline { 4 - 5 } & & SpkrtoMic1 (Top) & SprktoMic2 (Bot) \\
\hline \hline $1^{\text {st }}$ Structural & 80.4 & $78-82$ & 1.70 & 1.76 \\
$1^{\text {st }}$ Acoustic & 118.3 & $118-122$ & 6.75 & 6.77 \\
$2^{\text {nd }}$ Acoustic & 173.9 & $170-175$ & 0.66 & 0.00 \\
$2^{\text {nd }}$ Structural & 280.7 & $275-283$ & 1.28 & 1.72 \\
\hline \multicolumn{2}{l|}{ Broadband $(40-1000 \mathrm{~Hz})$} & 1.06 & 0.99 \\
\hline
\end{tabular}

Table 5.3: SLC Controller (Both On) Performance Summary

\begin{tabular}{l|l|l||r|r}
\hline Mode & \multirow{2}{*}{\begin{tabular}{l} 
Frequency \\
\cline { 3 - 4 }
\end{tabular}} & \multicolumn{1}{|c||}{ Reduction } & \multicolumn{2}{|c}{ Reduction (dB) } \\
\cline { 4 - 5 } & Range (Hz) & SpkrtoMic1 (Top) & SprktoMic2 (Bot) \\
\hline \hline $1^{\text {st }}$ Structural & 80.4 & $78-82$ & 6.42 & 3.06 \\
$1^{\text {st }}$ Acoustic & 118.3 & $118-122$ & 9.61 & 8.19 \\
$2^{\text {nd }}$ Acoustic & 173.9 & $170-175$ & -9.51 & -2.21 \\
$2^{\text {nd }}$ Structural & 280.7 & $275-283$ & 7.25 & 2.63 \\
\hline Broadband (40-1000 Hz) & 2.08 & 1.37 \\
\hline
\end{tabular}



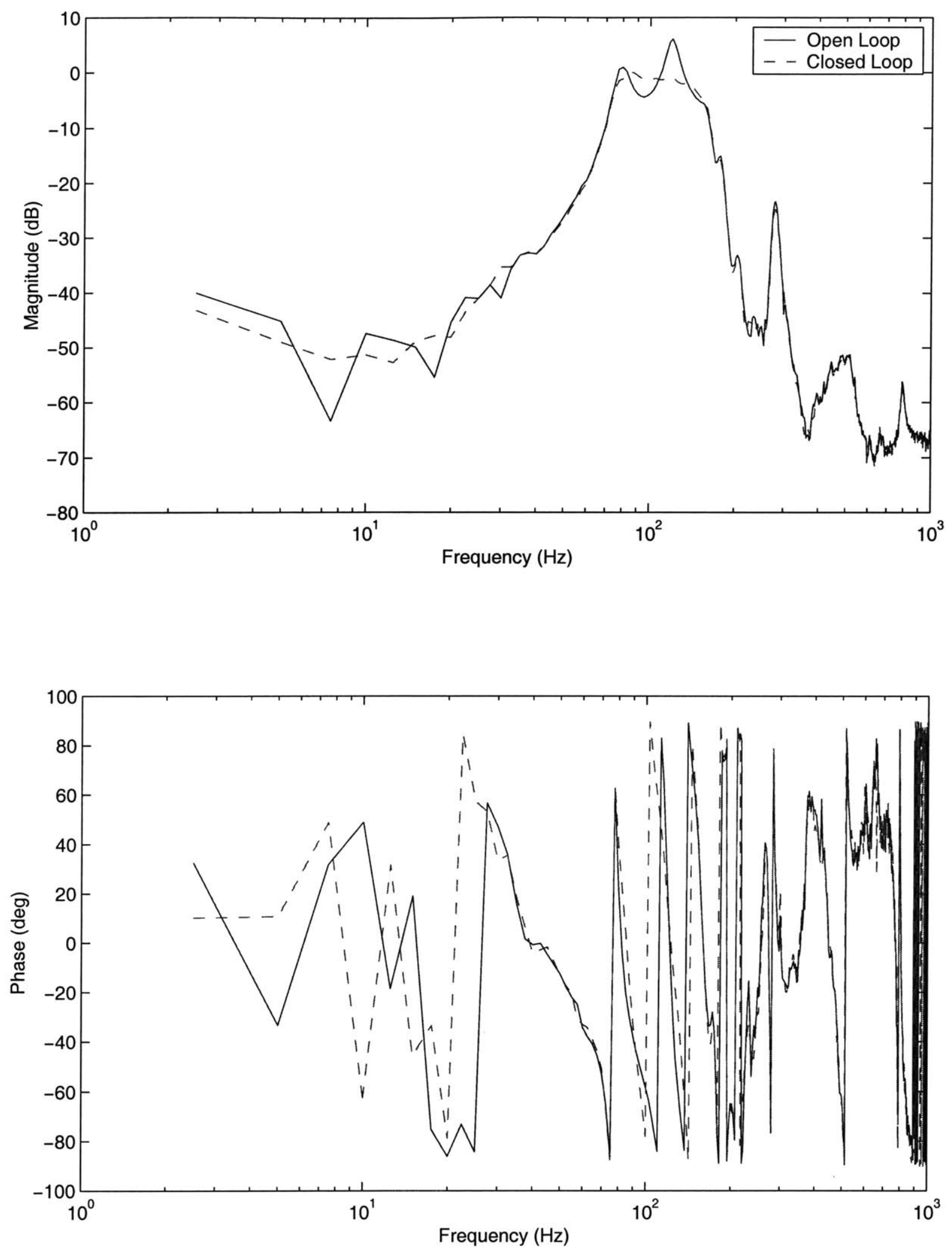

Figure 5-15: Successive Loop Closure Controller Performance (Bottom Plate System On Only) 

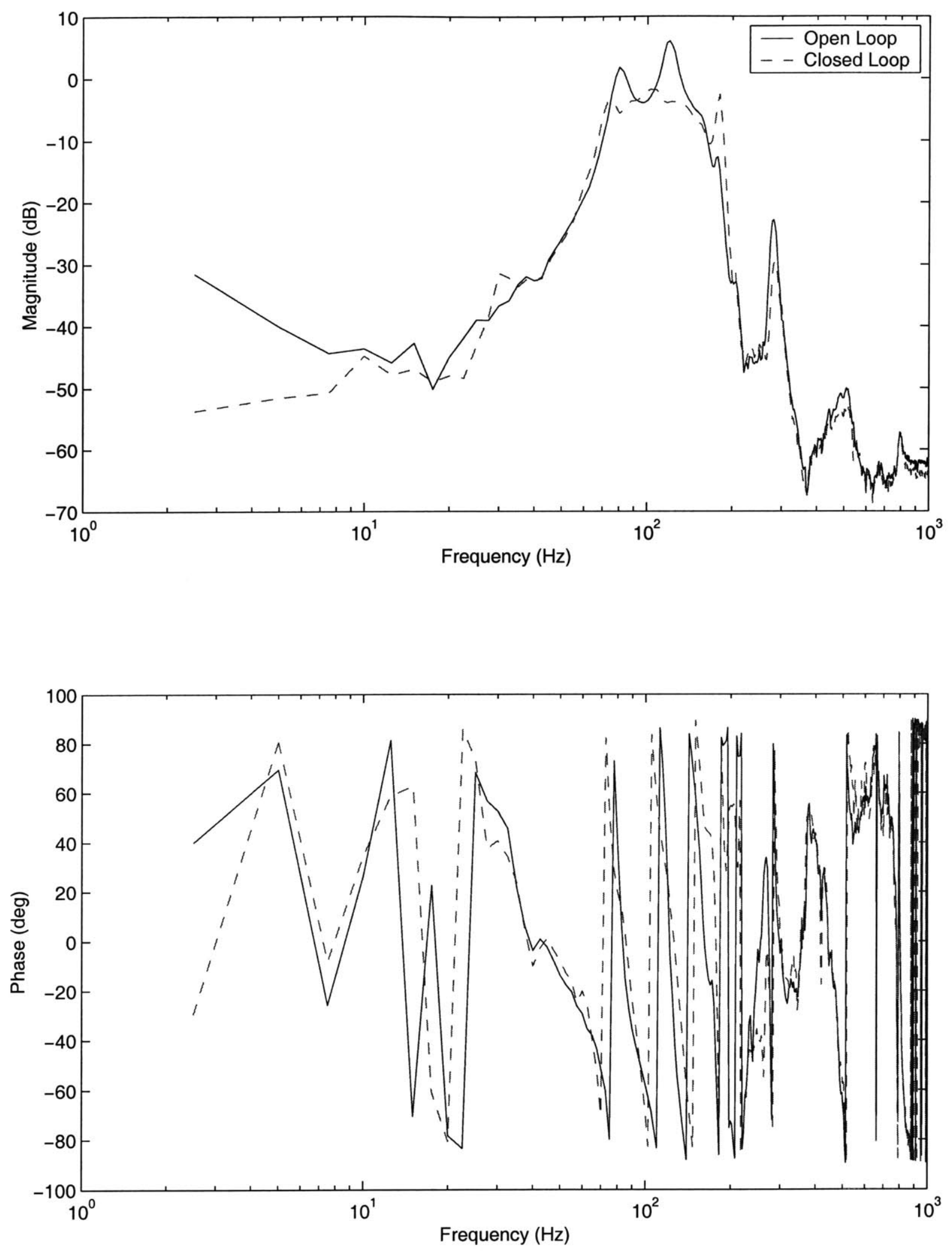

Figure 5-16: Successive Loop Closure Controller Performance (Both On) 


\subsubsection{Interference Controller}

The test chamber configuration used to test the Interference Controller was the Dual Layer Chamber Configuration shown in Figure 2-3. The control loop diagram for the Interference Controller is shown in Figure 5-17. The only difference between the Interference Controller and the Successive Loop Closure (SLC) Controller described in Section 5.2.2 was the order in which the plate controllers were designed. For the Interference Controller, the controller for the top plate system (farther from the disturbance source) was designed first and the controller for the bottom plate system (closer to the disturbance source) designed afterward. For the SLC Controller, the order was reversed. The goal of the Interference Controller was to minimize the broadband response from the disturbance speaker, which represents the volume outside the fairing, to Microphone 1, which represents the volume inside the fairing.

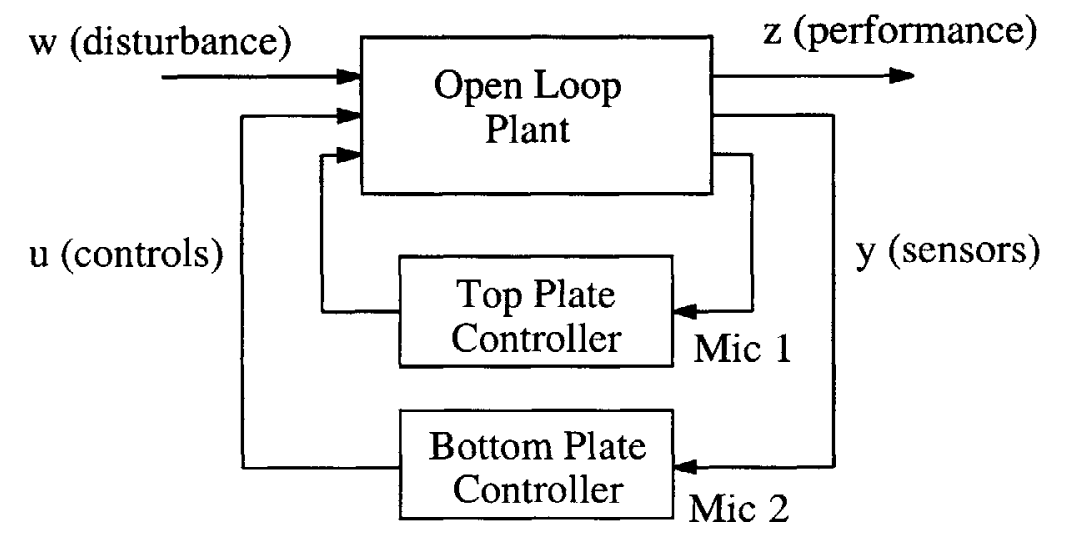

Figure 5-17: Interference Controller Configuration

\section{Open Loop}

The components of the test chamber used for this configuration are the same as those for the SLC Controller described in Section 5.2.2. Mic1 and Mic2 were the feedback and the performance sensors $(\boldsymbol{y}$ and $\boldsymbol{z}$ ). PZT1 and PZT2 were the actuators $(\boldsymbol{u})$, and the bottom speaker was the disturbance source $(\boldsymbol{w})$.

The Interference Control has two controllers with one input and one output each, which is similar to the SLC Controller configuration. Like the SLC Controller, the 
cross-coupling transfer functions (PZT1-to-Mic2 and PZT2-to-Mic1) were not included in the plant description, so that the sizes of the $G_{y u}$ and the $G_{y w}$ blocks were $1 \times 1$. The $G_{y u}$ and $G_{y w}$ transfer functions for the bottom plate compensator and for the top plate compensator are shown in Figures 5-18 and 5-19, respectively.

Mic2-to-Mic1 was used instead of Spkr-to-Mic1 for the top plate controller $\left(G_{y w}\right)$ because the acoustic cavity pressure acts as the originating noise source for the top plate. Therefore, the bottom plate, actively controlled or not, acts as a shaping filter for the disturbance source. Intuitively, Mic2-to-Mic1 is a better choice than Spkrto-Mic1 because Mic2-to-Mic1 describes how much energy is flowing through the top plate, which is the quantity that is to be minimized for active structural control. Although Spkr-to-Mic1 was not used in the plant description, it is the performance metric $(z)$ that describes how well the closed loop dual layer system is working compared to its open loop counterpart because it represents how much acoustic energy is flowing across both plates or the entire active structural system. The two performance metrics, Spkr-to-Mic1 and Spkr-to-Mic2, are shown in Figure 5-20.

The modes of the system are determined by examining the $G_{y w}$ transfer functions. Because the configuration was exactly the same as in the Successive Loop Closure Controller case, the Interference Controller's top and bottom plate systems also had modes at different frequencies. The top plate system had structural modes at $63.0 \mathrm{~Hz}$ and $275.6 \mathrm{~Hz}$ and had acoustic modes at $157.1 \mathrm{~Hz}$ and $206.6 \mathrm{~Hz}$. The bottom plate system had structural modes at $78.7 \mathrm{~Hz}$ and $284.1 \mathrm{~Hz}$ and had acoustic modes at $121.3 \mathrm{~Hz}$ and $179.0 \mathrm{~Hz}$. These values are moderately different than those for the SLC Controller, but the difference can be attributed to the fact that the bottom plate controller was active when the top plate open loop transfer function was taken for the SLC Controller and that the top plate controller was active when the bottom plate open loop transfer function was taken for the Interference Controller. The active structural plate was able to shift the acoustic and structural modes significantly. 

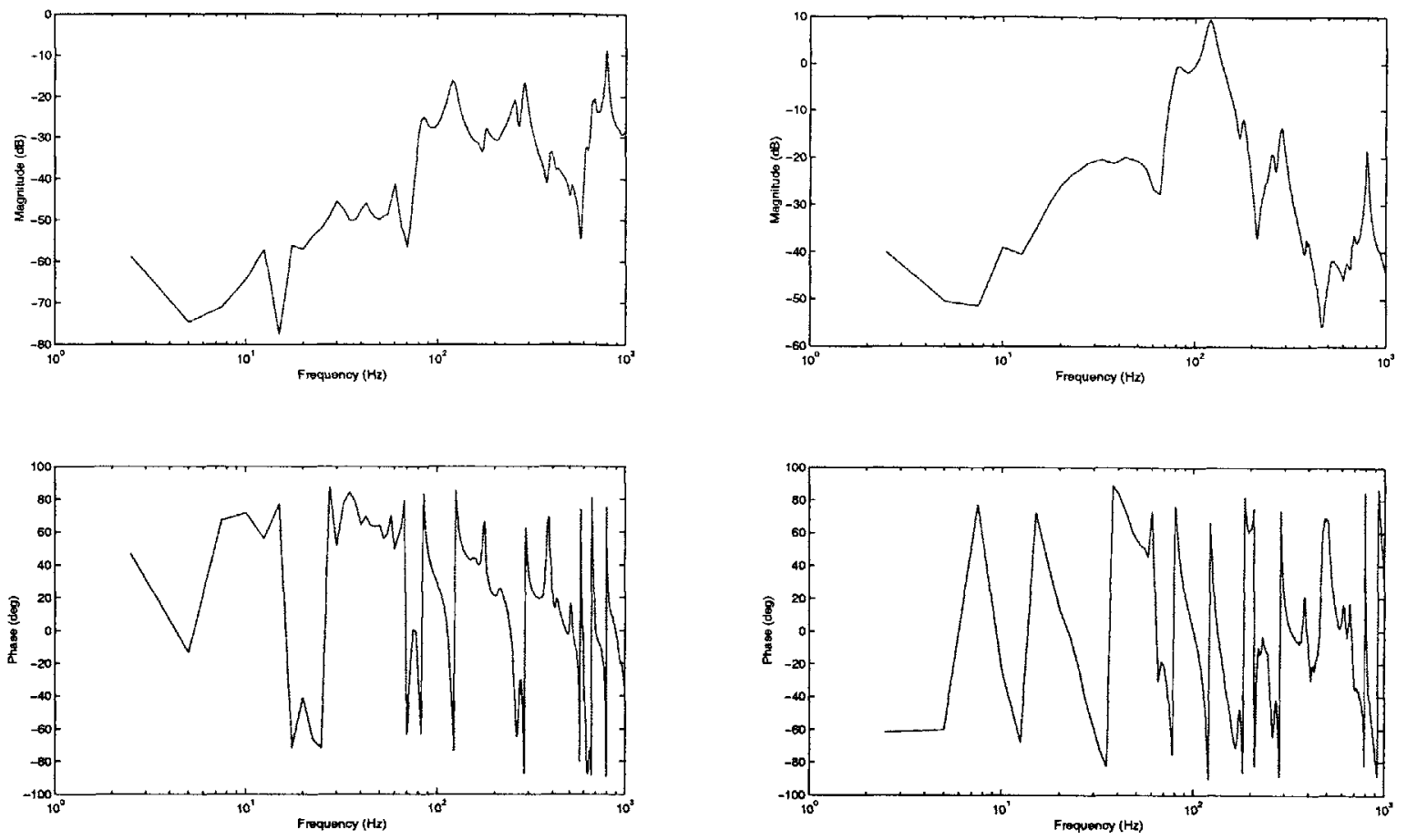

$G_{y u}:$ PZT2-to-Mic2

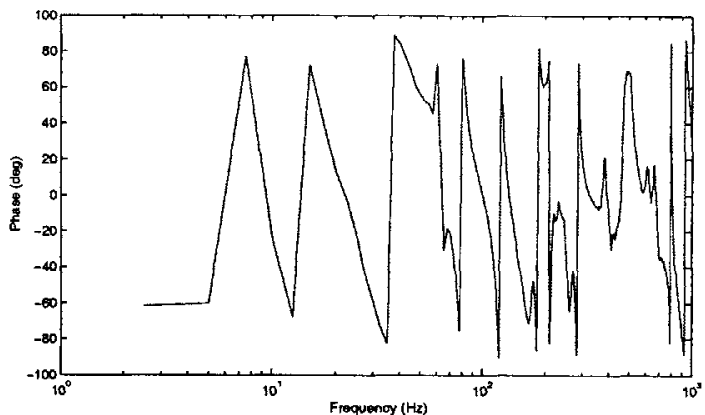

$G_{y w}:$ Spkr-to-Mic2

Figure 5-18: Interference Controller Bottom Plate Open Loop $G_{y u}$ and $G_{y w}$ Transfer Functions
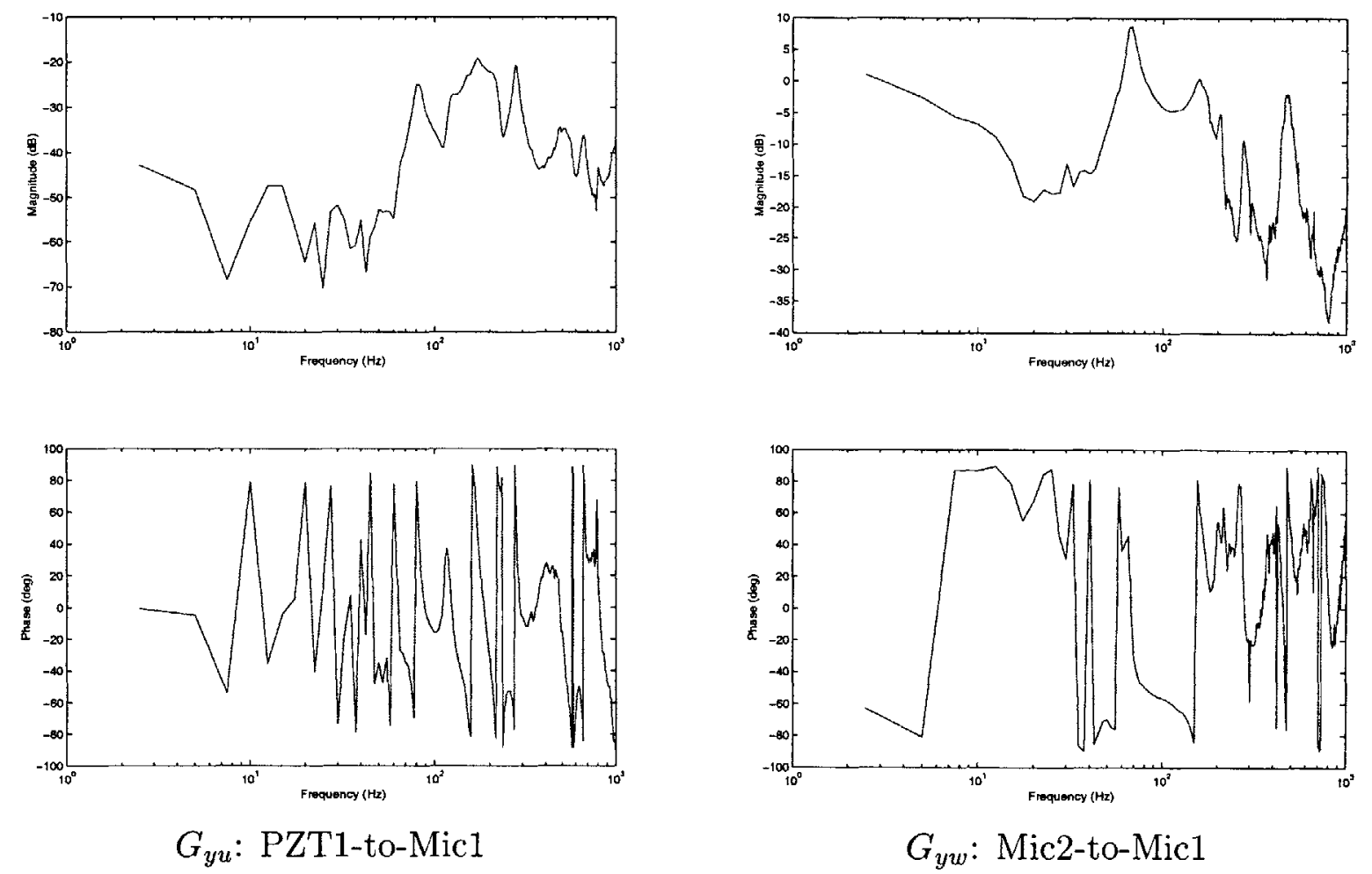

Figure 5-19: Interference Controller Top Plate Open Loop $G_{y u}$ and $G_{y w}$ Transfer Functions 

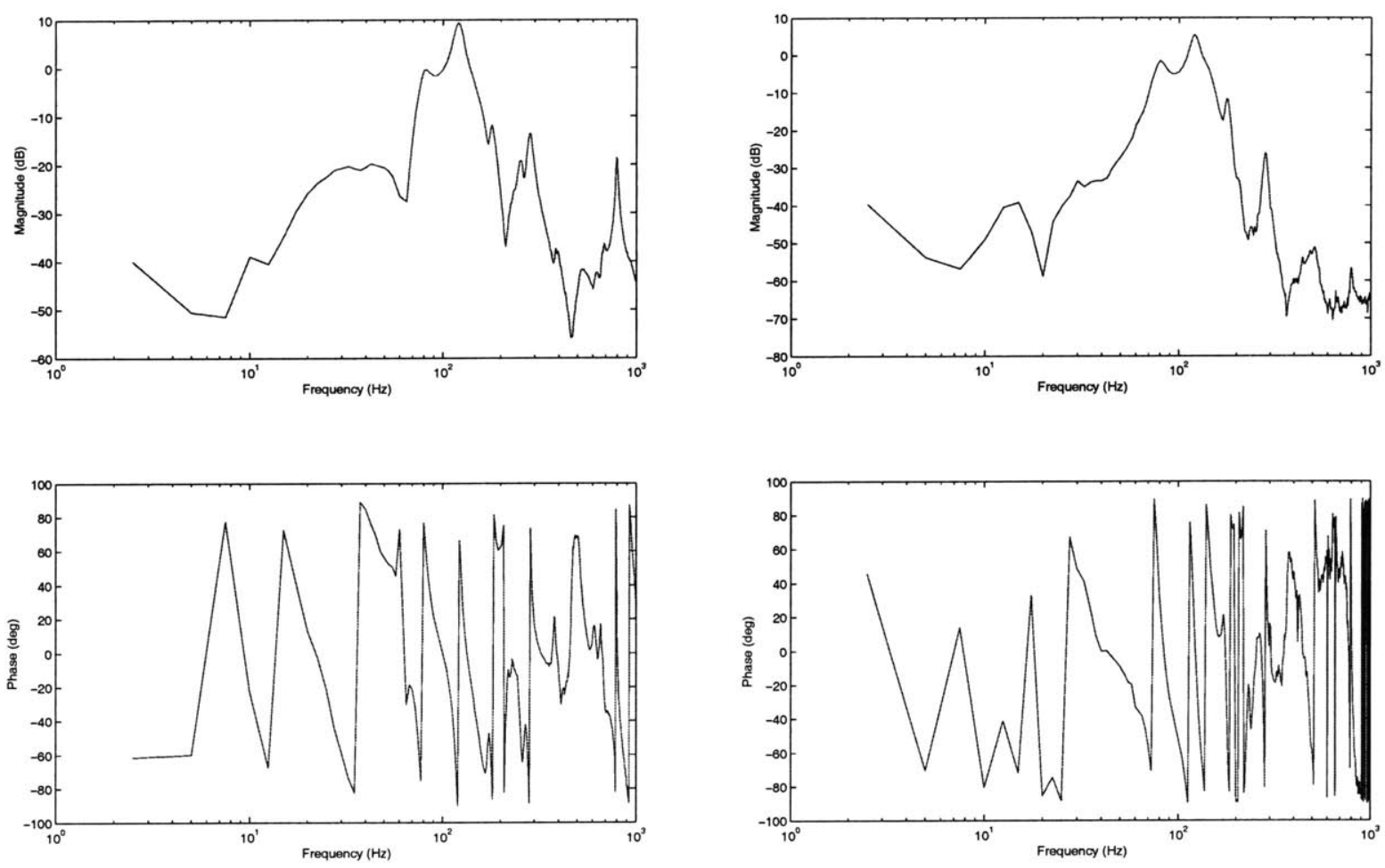

Bottom Plate $G_{z w}$ : Spkr-to-Mic2

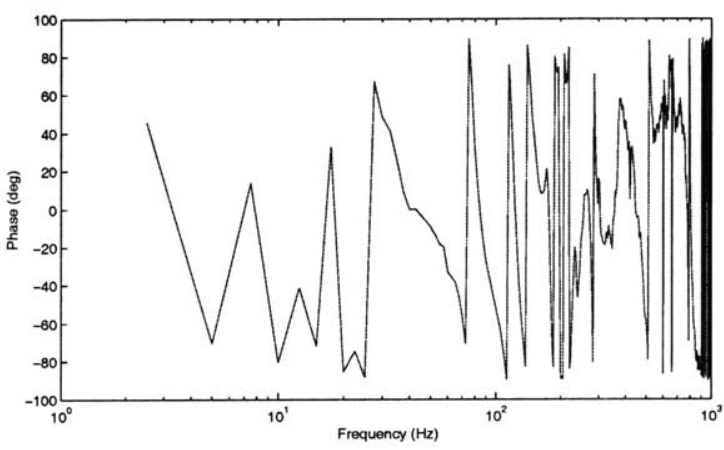

Top Plate $G_{z w}$ : Spkr-to-Mic1

Figure 5-20: Interference Controller Open Loop $G_{z w}$ Transfer Functions for Both Plates 


\section{Controller Design}

The compensator used for transmission control was designed using the LQG Method described in Chapter 4 and was implemented using a dSPACE digital computer operating at a sampling frequency of $5 \mathrm{kHz}$. The sizes of the bottom plate and top plate controllers were fifty-three and fifty-four states, respectively.

Figure 5-21 shows the compensator transfer functions. The controller responses are large at the frequencies where the corresponding states have been penalized by entering positive values in the $Q$ matrix in the LQR cost function. The top plate controller penalizes the modes at $63.0,157.1$ and $275.6 \mathrm{~Hz}$. The bottom plate controller penalizes the modes at 120.4, 121.3, 123.2, 179.0 and $284.1 \mathrm{~Hz}$. The peaks of $G_{u y}$ in Figure 5-21 correspond to these targeted modes, and they are easy to discern because the controller response is changing smoothly over the frequency ranges that are not in the neighborhood of the system poles.

Figure 5-22 shows the Nichols plot that is derived from the loop transfer function formed by interconnecting the compensator and the open loop plant, $G_{y u}$. The bottom and top plate systems have gain and phase margins of $8 \mathrm{~dB}$ and $100^{\circ}$, and $12 \mathrm{~dB}$ and $110^{\circ}$, respectively.

\section{Closed Loop Performance}

The Interference Controller was designed to be the reverse of what the SLC Controller represented. Whereas the SLC Controller requires the bottom plate controller to be designed first and then the top plate controller second, the Interference Controller requires the reverse- the top plate controller is designed first and then the bottom plate controller is designed. The reversal of the design order was introduced because the top plate controller of the SLC Controller had a very low gain margin. By designing the bottom plate controller while the top plate was active, it was hoped that the bottom plate could increase system performance without causing the top plate controller to become unstable. The results obtained from implementing the Interference Controller was worse than the MIMO and SLC Controllers. 

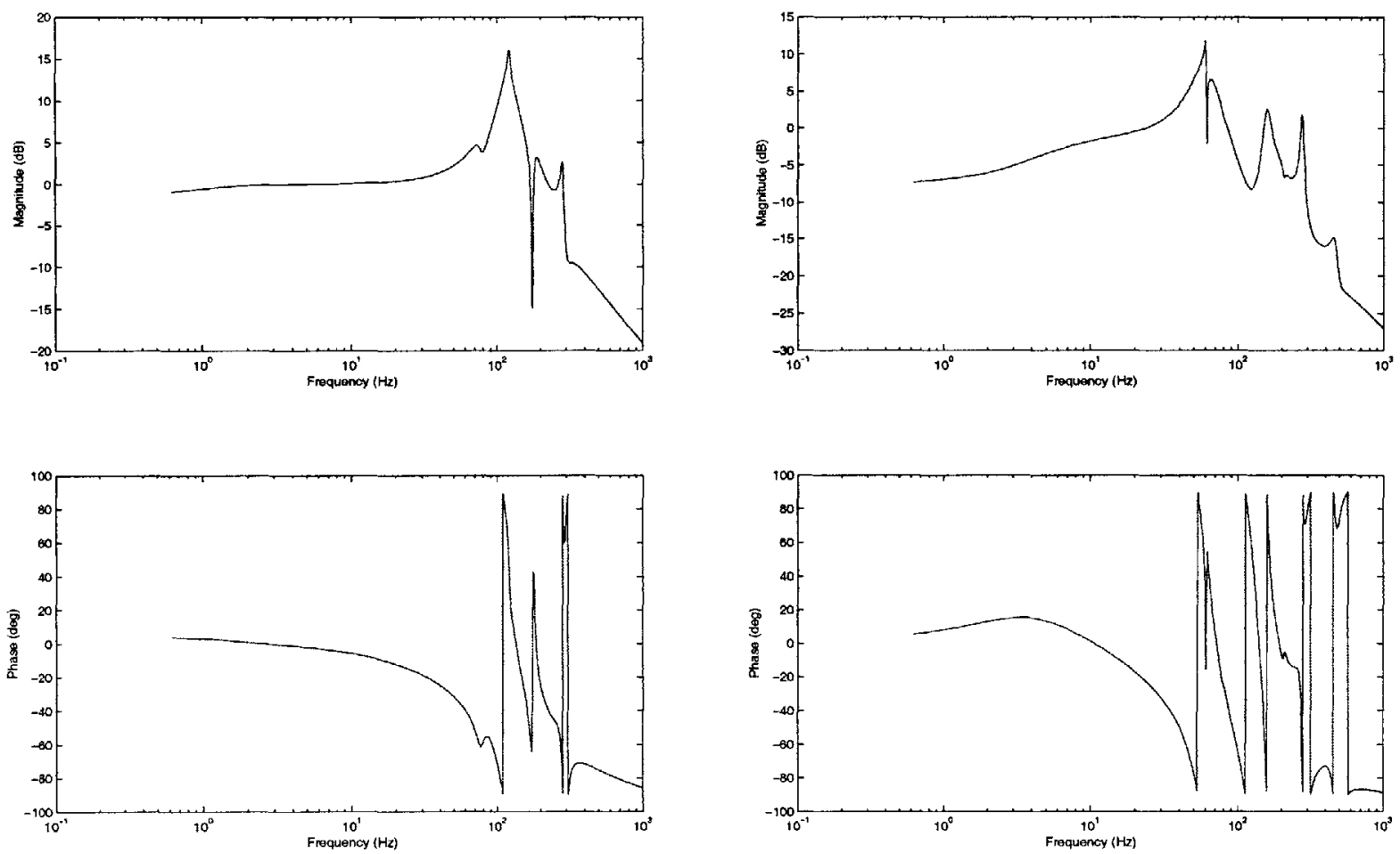

Bottom Plate $G_{u_{2} y_{2}}$ : Mic2-to-PZT2

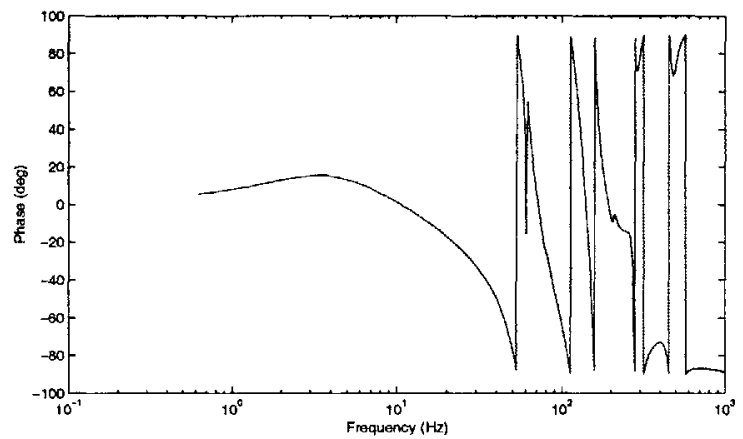

Top Plate $G_{u_{1} y_{1}}$ : Mic1-to-PZT1

Figure 5-21: Interference Controller $G_{u y}$ Transfer Functions 


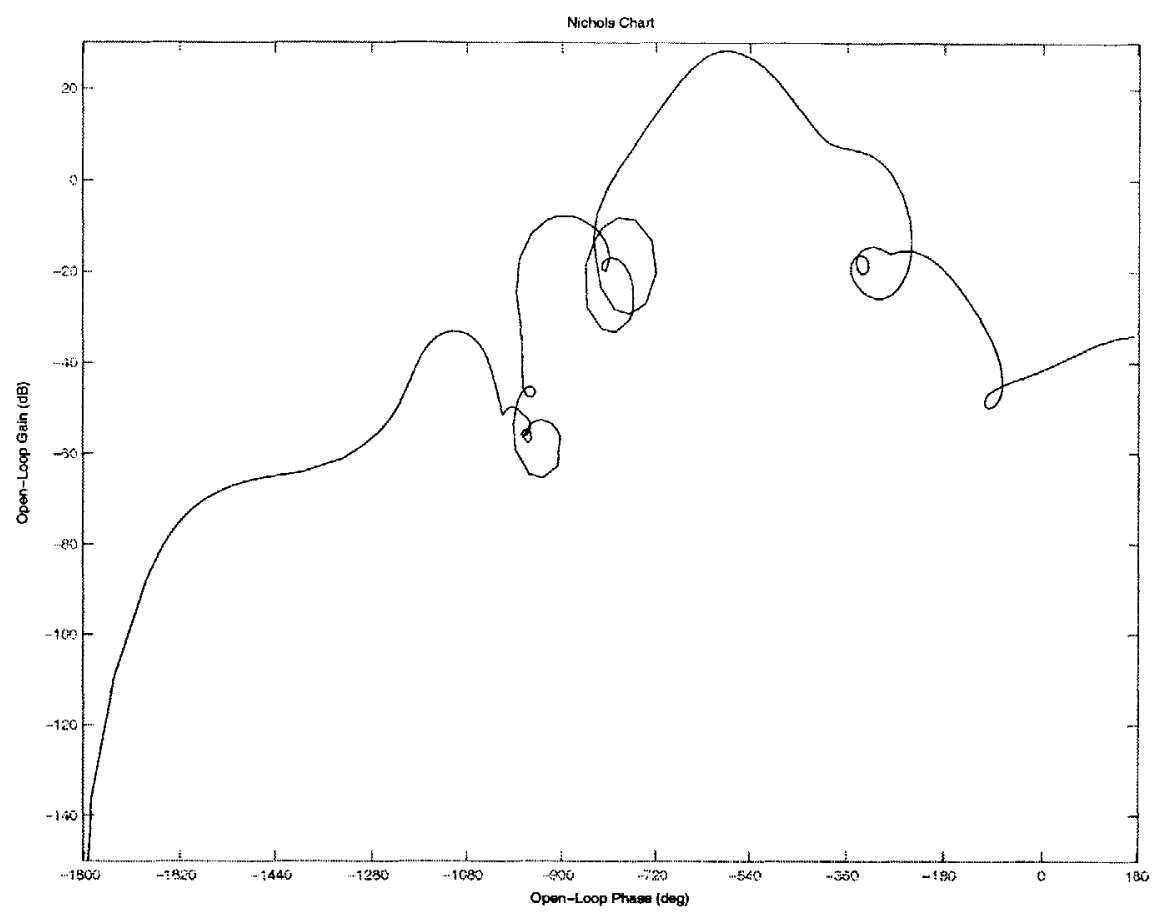

Bottom Plate System

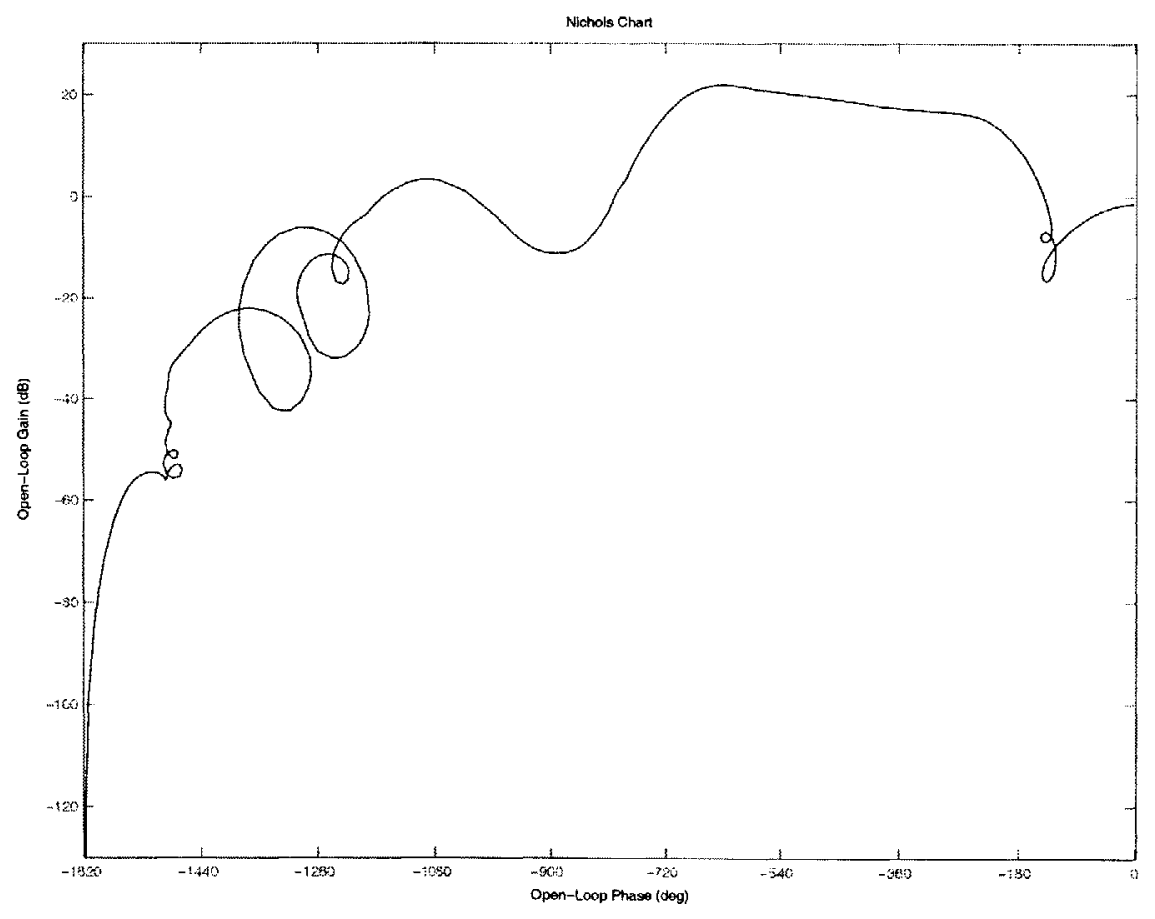

Top Plate System

Figure 5-22: Interference Controller Nichols Chart 
Figures 5-23 and 5-24 show the open and closed loop transfer functions of the Interference Controller. Figure 5-23 shows the system performance metric, Spkr-toMic1, when only the top plate is active, and Figure 5-24 shows the same performance metric when both plates are active. On its own, the top plate controller was not very effective compared the SLC Controller with only the bottom plate active. The top plate controller was able to reduce the first structural mode by $2.25 \mathrm{~dB}$ and the second structural mode by $1.11 \mathrm{~dB}$, but it was unable to have much of an effect on the first and second acoustic modes, which increased by $0.41 \mathrm{~dB}$ and $0.43 \mathrm{~dB}$ respectively. The reduction over the broadband range of $40-1000 \mathrm{~Hz}$ was $0.3041 \mathrm{~dB}$. The top plate controller did have a better gain margin at $8 \mathrm{~dB}$ than its SLC counterpart at $4 \mathrm{~dB}$, which was confirmed by a greater range of stable gains. However, the overall performance was much less than its SLC Controller counterpart. A summary of the Interference Controller using only the top plate controller can be found in Table 5.4.

Designing and implementing the bottom plate controller improved performance significantly, but the final results were still worse than the MIMO and SLC Controllers. With both plate controllers active, the first structural and acoustic modes were reduced by $0.43 \mathrm{~dB}$ and $10.249 \mathrm{~dB}$, respectively. The second acoustic mode, as in the SLC Controller case, increased dramatically at $7.98 \mathrm{~dB}$, and the second structural mode was reduced by $5.53 \mathrm{~dB}$. The same stability issues that caused the SLC Controller to become unstable also caused the Interference Controller to become unstable. Overall, the transmission of acoustic energy through the system over the range of $40-1000 \mathrm{~Hz}$ was reduced by $1.06 \mathrm{~dB}$. Table 5.5 summarizes the results obtained when both plates were actively controlled. 
Table 5.4: Interference Controller (Top Only) Performance Summary

\begin{tabular}{l|l|l||r|r}
\hline Mode & Frequency & Reduction & \multicolumn{2}{|c}{ Reduction (dB) } \\
\cline { 4 - 5 } & $(\mathrm{Hz})$ & Range $(\mathrm{Hz})$ & SpkrtoMic1 (Top) & SprktoMic2 (Bot) \\
\hline \hline $1^{\text {st }}$ Structural & 81.9 & $80-85$ & 2.25 & 1.43 \\
$1^{\text {st }}$ Acoustic & 123.2 & $118-122$ & -0.41 & 0.10 \\
$2^{\text {nd }}$ Acoustic & 184.8 & $170-175$ & -0.43 & -0.70 \\
$2^{\text {nd }}$ Structural & 284.1 & $275-283$ & 1.10 & 0.88 \\
\hline \multicolumn{2}{l|}{ Broadband (40-1000 $\mathrm{Hz})$} & 0.30 & 0.23 \\
\hline
\end{tabular}

Table 5.5: Interference Controller (Both On) Performance Summary

\begin{tabular}{l|l|l||r|r}
\hline Mode & \multirow{2}{*}{$\begin{array}{l}\text { Frequency } \\
\end{array}$} & Reduction & \multicolumn{2}{|c}{ Reduction (dB) } \\
\cline { 5 - 5 } & $(\mathrm{Hz})$ & Range $(\mathrm{Hz})$ & SpkrtoMic1 (Top) & SprktoMic2 (Bot) \\
\hline \hline $1^{\text {st }}$ Structural & 81.9 & $80-85$ & 0.42 & -0.99 \\
$1^{\text {st }}$ Acoustic & 118.3 & $118-122$ & 10.25 & 10.86 \\
$2^{\text {nd }}$ Accustic & 173.9 & $170-175$ & -7.98 & -2.15 \\
$2^{\text {nd }}$ Structural & 280.7 & $275-283$ & 5.53 & 4.94 \\
\hline \multicolumn{2}{l|}{ Broadband $(40-1000 \mathrm{~Hz})$} & 1.06 & 0.93 \\
\hline
\end{tabular}

\subsubsection{Power Diode Controller}

The test chamber configuration used to test the Power Diode Controller was the Diode Chamber Configuration shown in Figure 2-4. The control loop diagram for the Diode Controller is shown in Figure 5-25. The bottom plate controller was designed first, and then the top plate controller was designed using an actively controlled bottom plate as its open loop plant because the broadband performance of the SLC Controller was much better than the Interference Controller. Although the SLC Controller performed better than the Interference Controller, its severe amplification of the $201.3 \mathrm{~Hz}$ acoustic mode response limited the gain and the performance of the overall compensator. In an attempt to limit the acoustic coupling between the plates and to reduce the magnitude of the $201.3 \mathrm{~Hz}$ acoustic mode, a Power Diode was implemented on the bottom plate. When Carlos Guiterrez implemented the Power Diode in his experiments [12], he only targeted the first structural mode. In this configuration, the Power Diode was set to target several structural and acoustic 

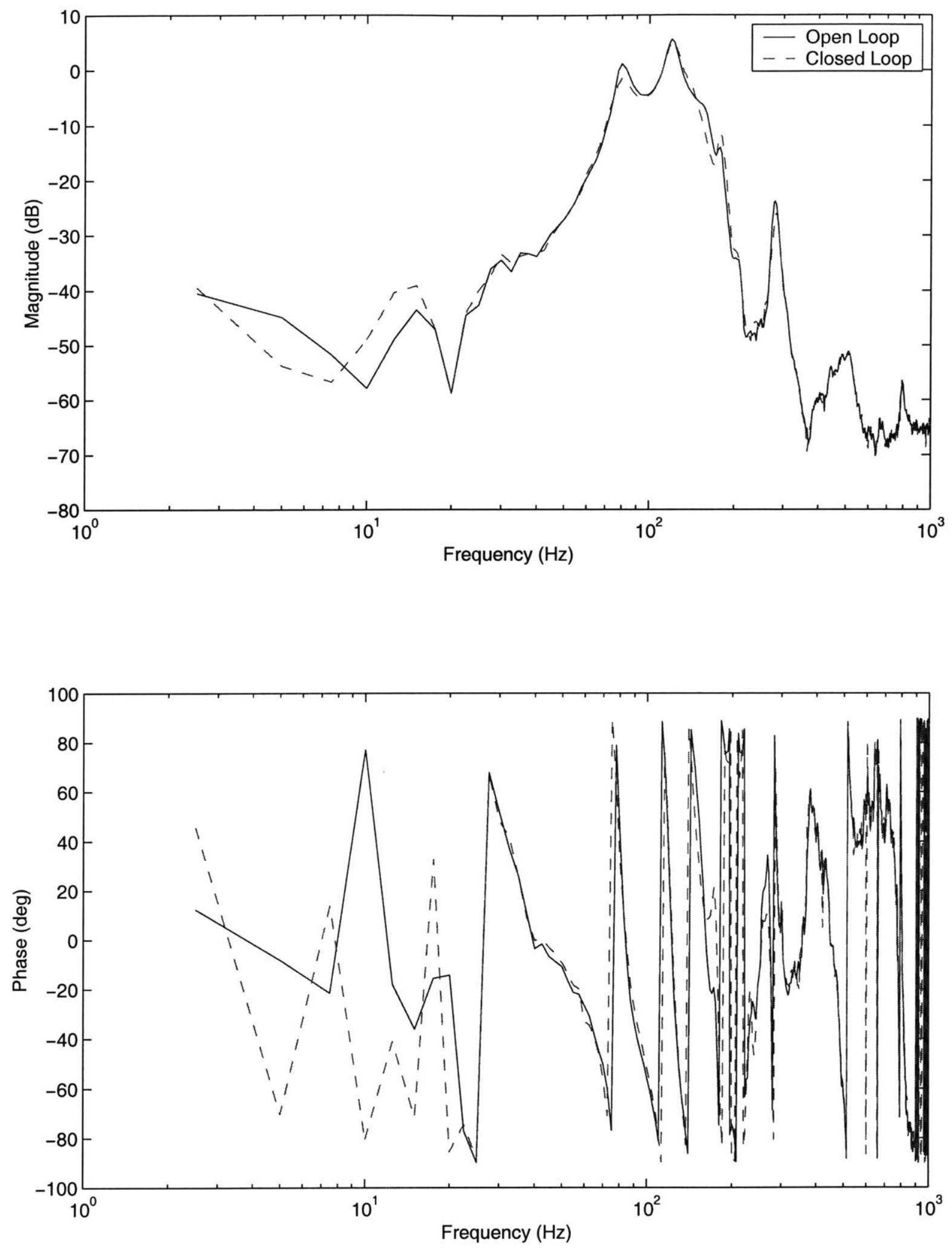

Figure 5-23: Interference Controller Performance (Top Plate System On Only) 

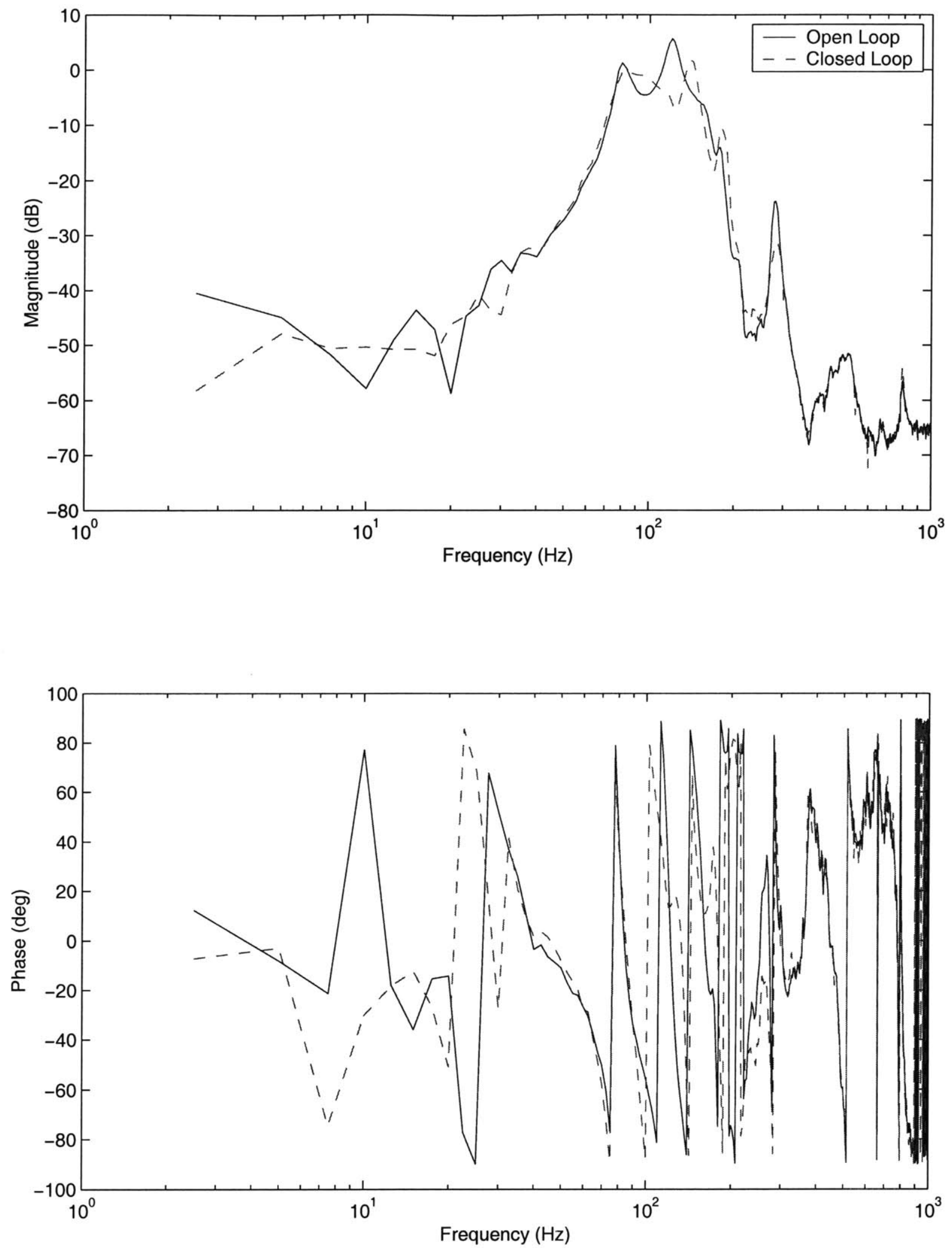

Figure 5-24: Interference Controller Performance (Both On) 


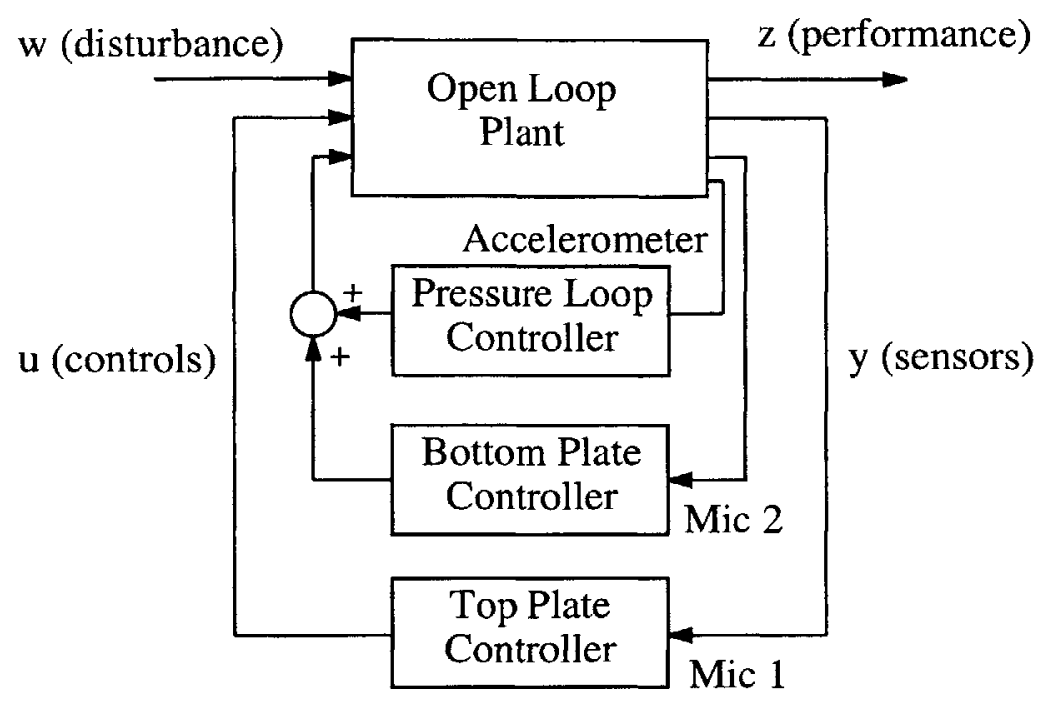

Figure 5-25: Diode Controller Configuration

modes, and the performance metric was to minimize the energy flow over the 40$1000 \mathrm{~Hz}$ range, not just at the first structural mode. The top plate system controller was designed as before.

\section{Open Loop}

The components of the test chamber used for this configuration are the following. Mic1, Mic2, Mic3, Mic4 and the accelerometer were the feedback and the performance sensors $(\boldsymbol{y}$ and $\boldsymbol{z})$. PZT1 and PZT2 were the actuators $(\boldsymbol{u})$, and the top and bottom speakers were the disturbance sources $(\boldsymbol{w})$. The bottom speaker was used as the disturbance source to which both plate system controllers were tuned to, and the top speaker was used to verify and measure how well the diode performed in the reverse direction. The addition of the top end cap (with disturbance speaker) changed the acoustic characteristics of the system, but it was preferable to swapping the location of the two plates and using the bottom disturbance speaker only because modifying the test chamber physically often leads to slightly different system characteristics and slightly different transfer functions.

The top plate controller was designed in the same manner as for the SLC Controller, and its $G_{y u}$ and $G_{y w}$ blocks was $1 \times 1$. In contrast, the bottom plate controller's $G_{y u}$ and $G_{y w}$ blocks were $2 \times 1$ where the two outputs are Mic3 and the accelerometer, 
which is affixed to the bottom surface of the bottom plate. Note that Mic3 was used instead of Mic2 as a $G_{y u}$ transfer function because the accelerometer and the feedback sensor microphone had to have the same phase and, therefore, must be on the same side. As in the other configurations, Mic2 was the performance sensor for the bottom plate system.

As with the SLC Controller, the cross-coupling transfer functions (PZT1-to-Mic2 and PZT2-to-Mic1) were not included in the plant description. Figures 5-26 and 5-27 show the $G_{y u}$ and $G_{y w}$ transfer functions for the bottom plate system, respectively. Figure 5-28 shows the $G_{y u}$ and $G_{y w}$ transfer functions for the top plate system, and Figure 5-29 shows the $G_{z w}$ transfer functions for both plate systems. It is interesting to note that the magnitudes of the $G_{y u}$ and $G_{y w}$ transfer functions for the top plate are smaller than their SLC Controller counterparts. The cause of the discrepancy can be traced to the presence of the top end cap (with speaker), which, because of the viscous effects of the enclosed fluid, caused the air within the top cavity to act "stiffer" and to be much more effective in damping out the vibration of the top plate.

The modes of the system are determined by examining the $G_{y w}$ transfer functions. The configuration included the top end-cap of the test chamber, which changed the modal frequencies of the system slightly. The top plate had structural modes at 92.6 $\mathrm{Hz}$ and $284.8 \mathrm{~Hz}$ and had acoustic modes at $124.9 \mathrm{~Hz}$ and $200.4 \mathrm{~Hz}$. The bottom plate system had structural modes at $92.3 \mathrm{~Hz}$ and $286.0 \mathrm{~Hz}$ and had acoustic modes at $126.1 \mathrm{~Hz}$ and $193.9 \mathrm{~Hz}$. The acoustic and structural modes of the two plate systems are a lot closer to each other because both systems were surrounded by fully enclosed volumes, in contrast to the other test chamber configurations where the top chamber is open to the atmosphere.

\section{Controller Design}

The compensator used for the transmission control was designed using the LQG Method described in Chapter 4 and was implemented using a dSPACE digital computer operating at a sampling frequency of $5 \mathrm{kHz}$. The size of the bottom plate and the top plate controllers were fifty-two and fifty states, respectively. For the pressure 

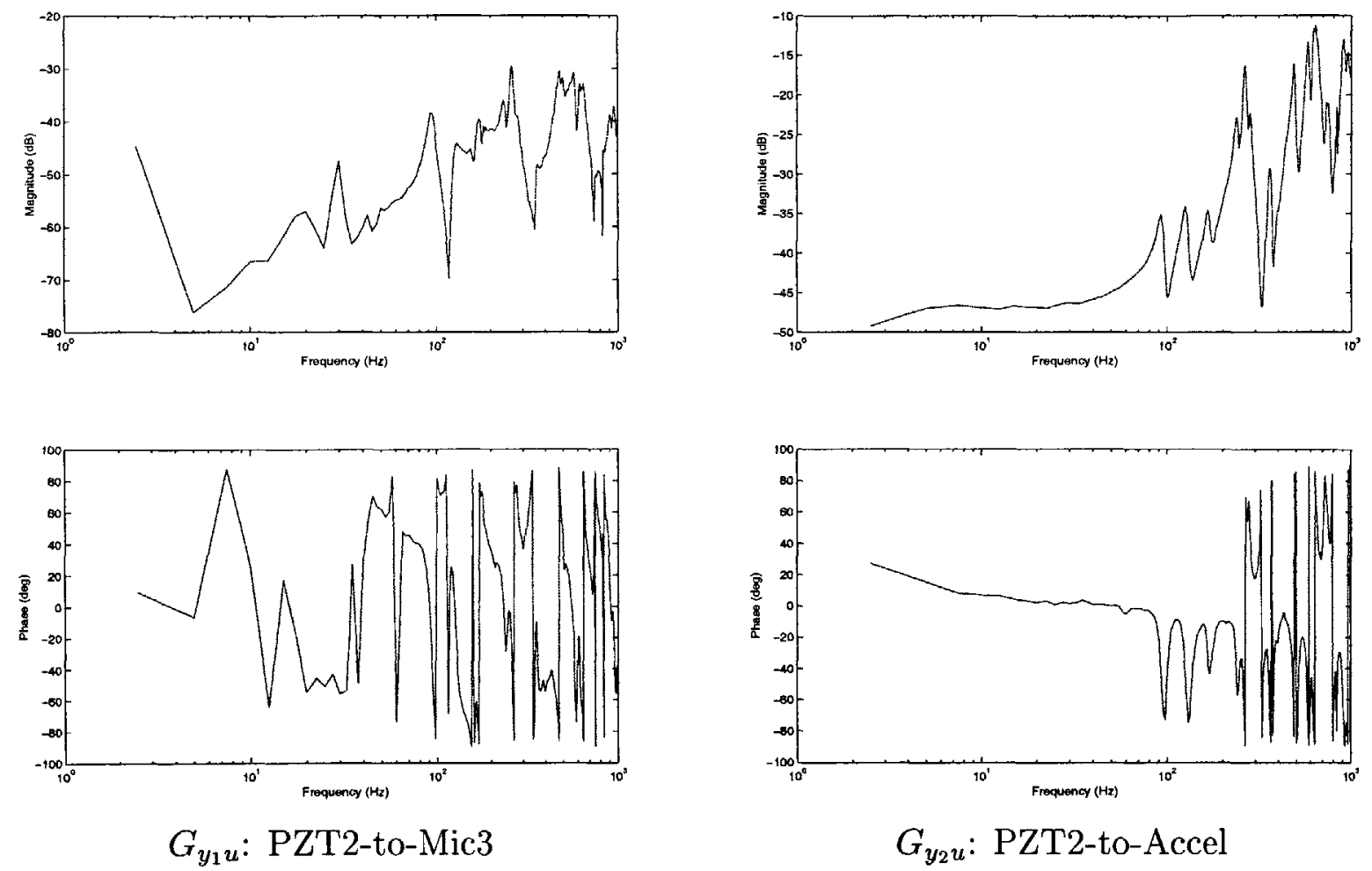

Figure 5-26: Diode Controller Bottom Plate Open Loop $G_{y u}$ Transfer Functions
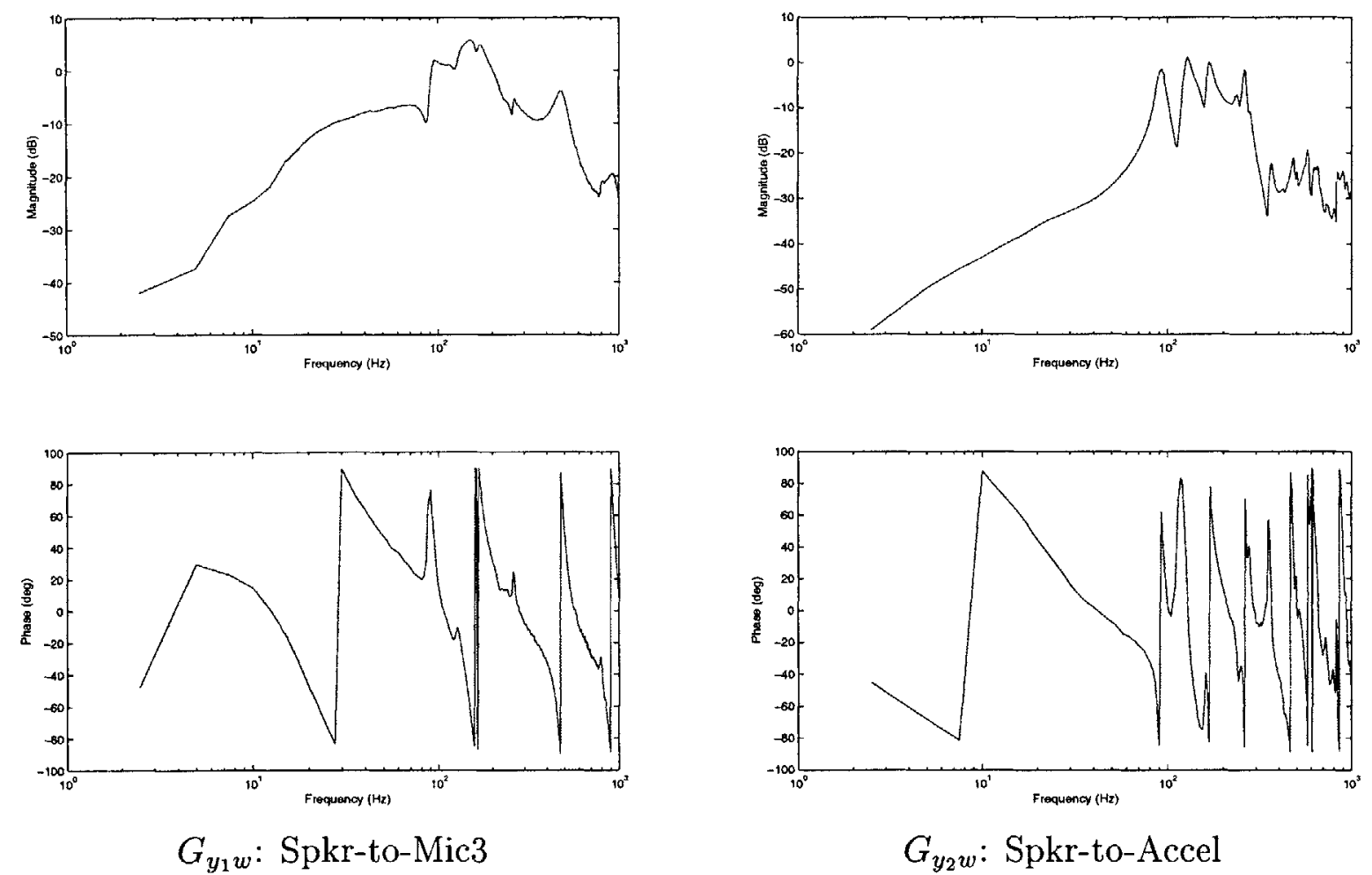

Figure 5-27: Diode Controller Bottom Plate Open Loop $G_{y w}$ Transfer Functions 

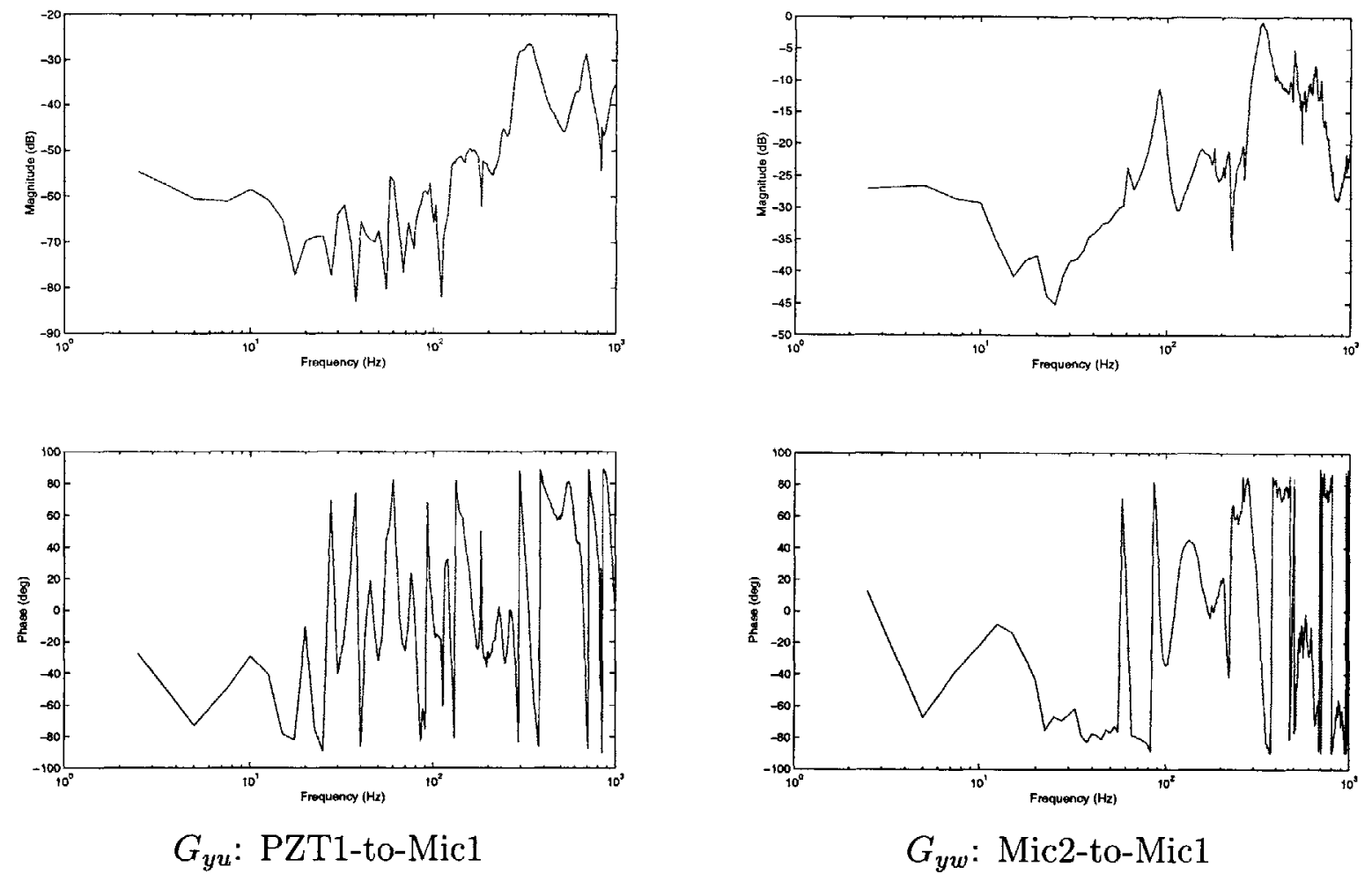

Figure 5-28: Diode Controller Top Plate Open Loop $G_{y u}$ and $G_{y w}$ Transfer Functions
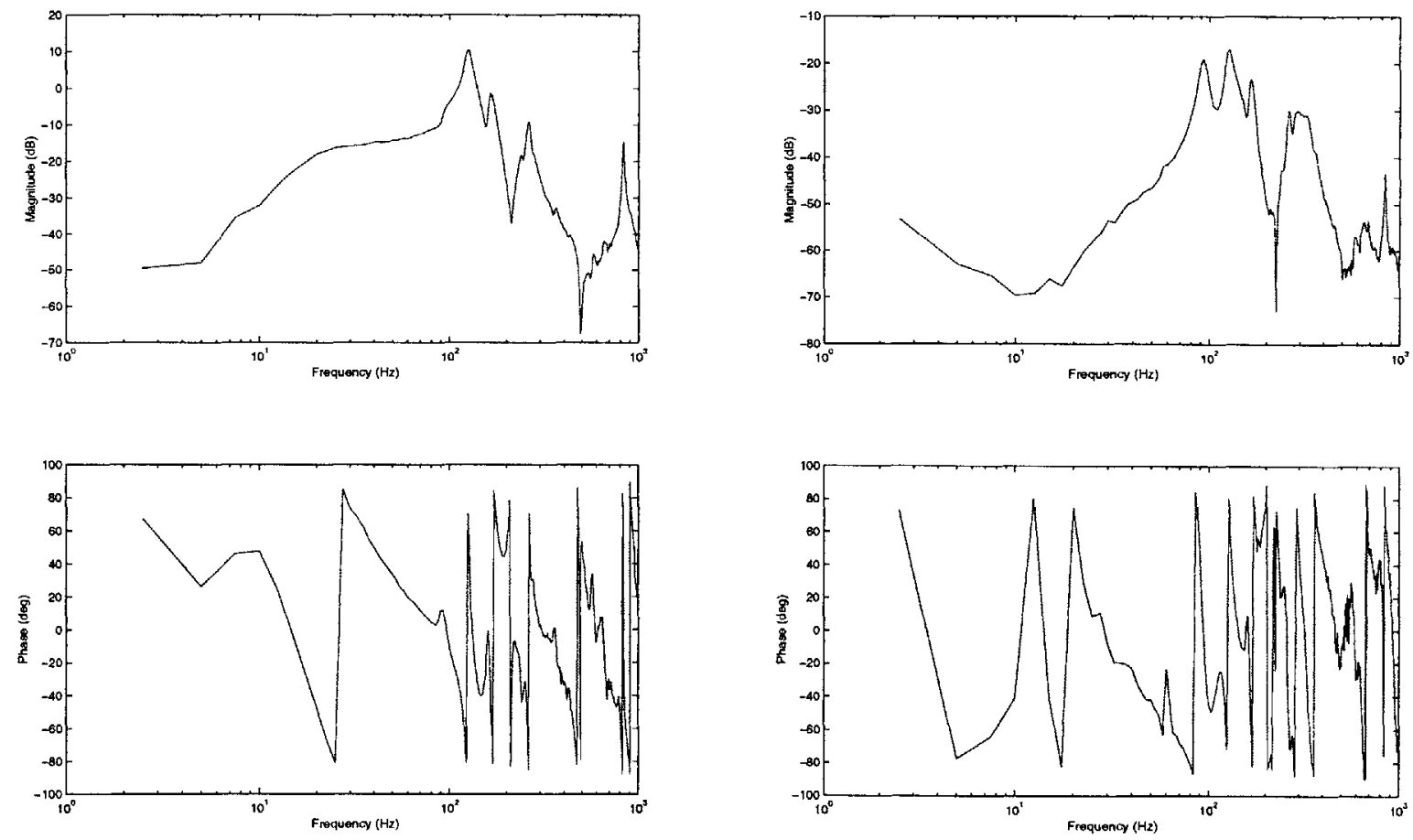

Bottom Plate $G_{z w}$ : Spkr-to-Mic2

Top Plate $G_{z w}:$ Spkr-to-Mic1

Figure 5-29: Diode Controller Open Loop $G_{z w}$ Transfer Functions for Both Plates 
loop, a high pass filter was applied to the accelerometer signal to eliminate the low frequency noise, and the resulting output was put through a discrete time integrator. The high pass filter was a fourth order Butterworth filter with a corner frequency at $30 \mathrm{~Hz}$. A significantly lower corner frequency would result in low frequency components of the accelerometer signal, which would cause the integrated signal to drift over time.

Figure 5-30 shows the compensator transfer functions. The controller responses are large at the frequencies where the corresponding states have been penalized by entering positive values in the $Q$ matrix in the LQR cost function. The bottom plate power diode controller penalizes the modes at 92.7, 126.1, 262.3, 286.0 and $832.5 \mathrm{~Hz}$. The top plate controller penalizes the modes at 92.6, 158.1, 244.3, 284.8, 336.4 and $781.3 \mathrm{~Hz}$. The peaks of $G_{u y}$ in Figure 5-13 correspond to the these targeted modes, and they are easy to discern because the controller response is changing smoothly over the frequency ranges that are not in the neighborhood of the system poles.

Figure 5-31 shows the Nichols plot that is derived from the loop transfer function formed by interconnecting the compensator and the open loop plant, $G_{y u}$. The bottom and top plate systems have gain and phase margins of $8 \mathrm{~dB}$ and $150^{\circ}$, and $12 \mathrm{~dB}$ and $30^{\circ}$, respectively.

\section{Closed Loop Performance}

The Power Diode Controller was implemented in response to the failure of the Interference Controller in stabilizing the second acoustic mode that was driving the system unstable. The Power Diode was implemented on the bottom plate, and it was hoped that the second acoustic mode's response could be stabilized by controlling the reflection characteristics of the bottom plate. As in the previous cases, the microphones were used for transmission control across the plate, but the diode had an additional sensor, an accelerometer. The signal from the accelerometer was sent through a high pass signal to eliminate the DC offset and then the specific force measurement was integrated to a velocity, which then could be used to characterize the pressure generated by the plate vibration. 

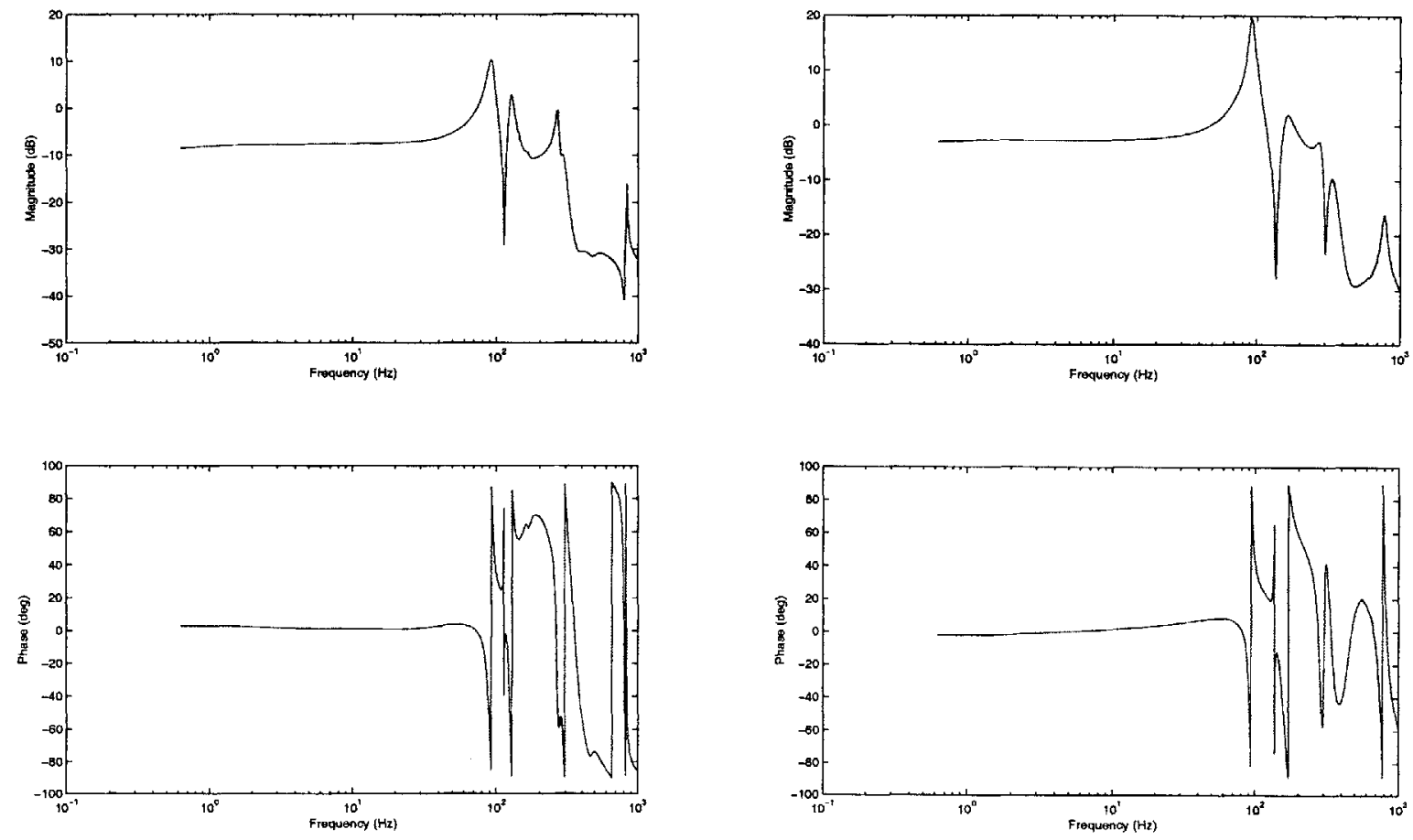

Bottom Plate $G_{u_{2} y_{2}}:$ Mic2-to-PZT2

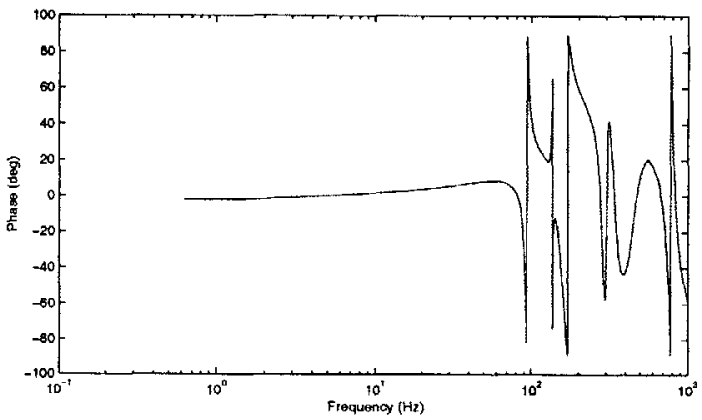

Top Plate $G_{u_{1} y_{1}}:$ Mic1-to-PZT1

Figure 5-30: Power Diode Controller $G_{u y}$ Transfer Functions 


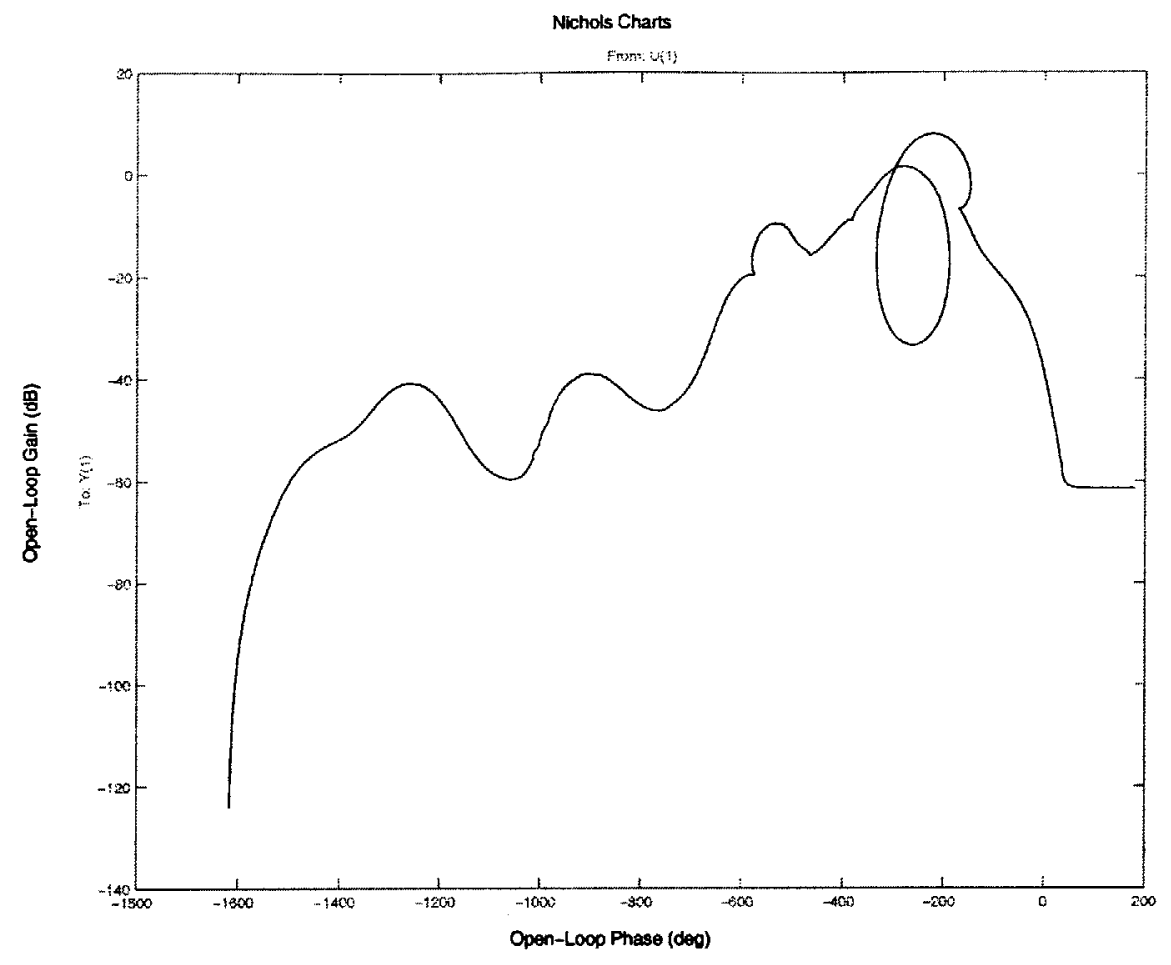

Bottom Plate System

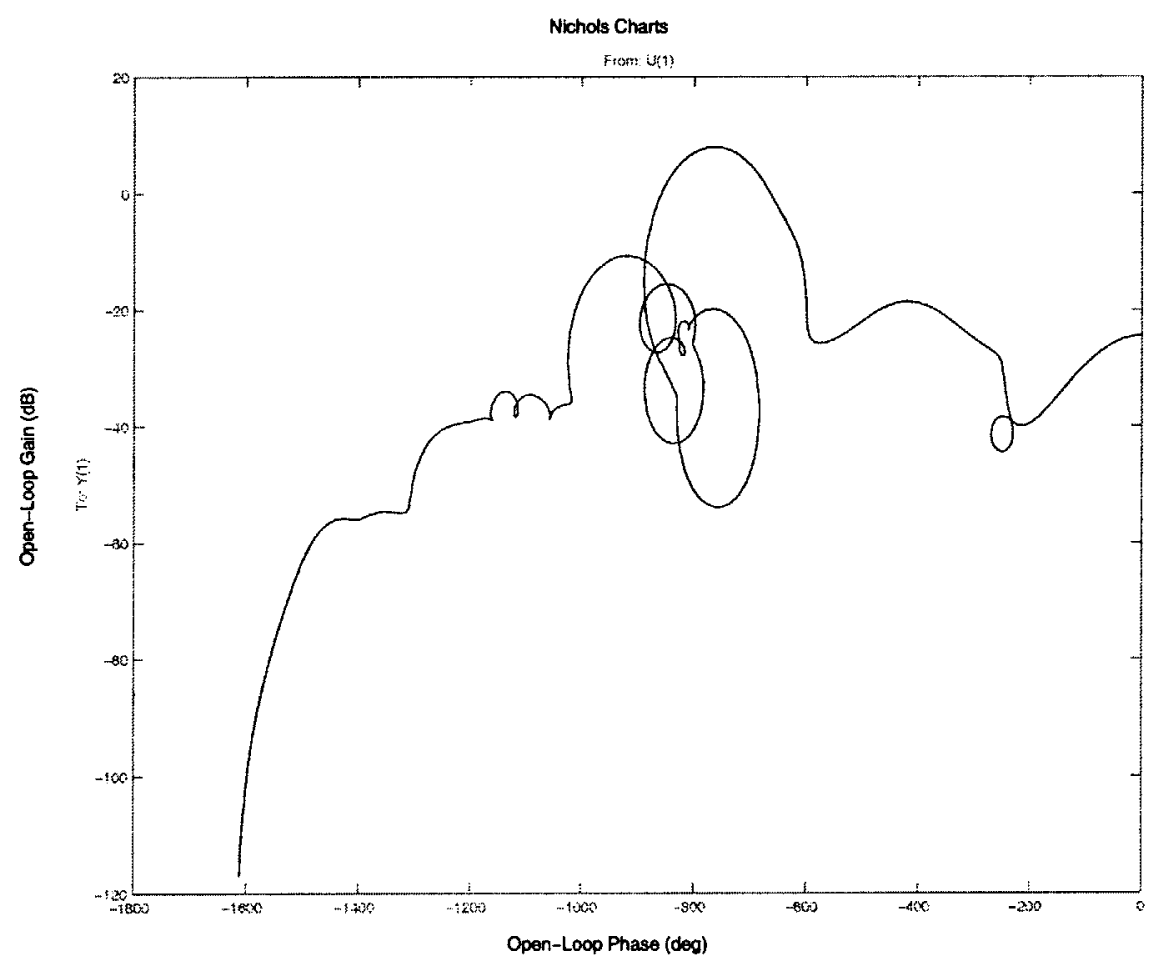

Top Plate System

Figure 5-31: Power Diode Controller Nichols Chart 
The integrated accelerometer signal was amplified and added to the bottom plate controller signal. The integrated accelerometer signal partially counteracts the bottom plate control signal, so that the efficacy of the overall transmission control system is reduced. Therefore, it is expected that the bottom plate controller would perform worse than its SLC Controller's counterpart. However, it was hoped that controller performance could be improved by preventing the second acoustic mode from becoming unstable.

Figures 5-32 and 5-33 show the open and closed loop transfer functions of the Power Diode Controller. Figure 5-32 shows the system performance metric, Spkr-toMic1, when only the bottom plate is active (including the pressure loop), and Figure 533 shows the same performance metric when both plates are active. As expected, the bottom plate controller was not as effective as its SLC Controller counterpart. The bottom plate controller of the Power Diode Controller posted $3.04 \mathrm{~dB}$ and $12.11 \mathrm{~dB}$ reductions on the first and second structural modes, respectively. It was less effective in suppressing the acoustic modal responses. The first acoustic mode was reduced by $1.49 \mathrm{~dB}$, and the second acoustic modal response increased slightly by $-0.50 \mathrm{~dB}$. The reduction over the broadband range of $40-1000 \mathrm{~Hz}$ was $0.46 \mathrm{~dB}$, compared to the 1.06 reduction obtained using the SLC Controller's bottom plate controller only. A summary of the Power Diode Controller using only the bottom plate controller can be found in Table 5.6 .

When the top plate controller was activated, the performance of the overall Power Diode Controller improved significantly. The first structural and acoustic modes were reduced by $6.99 \mathrm{~dB}$ and $1.92 \mathrm{~dB}$, respectively, compared to the $3.04 \mathrm{~dB}$ and $1.49 \mathrm{~dB}$ reductions obtained by using the bottom plate controller only. The performance of the second acoustic mode also improved from an increase of $0.50 \mathrm{~dB}$ to a very slight increase of $0.01 \mathrm{~dB}$. However, the attenuation of the second structural mode was reduced from $12.11 \mathrm{~dB}$ to $5.37 \mathrm{~dB}$. The fact that the second acoustic mode did not "spike" as it had in the SLC and Interference Controllers prove that the Power Diode configuration was effective in neutralizing some of the unstablizing effects. However, the gain in stability had a cost. The Power Diode Controller was only able to achieve 
a $1.06 \mathrm{~dB}$ reduction over the $40-1000 \mathrm{~dB}$ broadband range, which was worse than the results achieved using the MIMO and SLC Controllers and was comparable to the results achieved using the Interference Controller. Table 5.7 summarizes the results obtained when both plates were actively controlled.

Figure 5-34 shows the open and closed loop transfer functions taken using the top speaker as the disturbance source instead of the bottom speaker. This configuration tests how well the power diode is working because it is designed to minimize the amount of acoustic energy transmitted across the plate in one direction and to allow the acoustic energy to cross the plate from the other direction freely. As stated previously, the diode was able to reduce the transmission of energy in the "normal" configuration with the disturbance source at the bottom. And now that the top speaker is used as the disturbance source, the power diode actually improved the flow of acoustic energy through the system by $0.20 \mathrm{~dB}$. Therefore, the power diode proved to be working properly.

Table 5.6: Power Diode Controller (Bottom Only) Performance Summary

\begin{tabular}{l|l|l||r|r}
\hline Mode & \multirow{2}{*}{$\begin{array}{l}\text { Frequency } \\
\text { (Hz) }\end{array}$} & $\begin{array}{l}\text { Reduction } \\
\text { Range }(\mathrm{Hz})\end{array}$ & \multicolumn{2}{|c}{ Reduction (dB) } \\
\cline { 4 - 5 } & & SpkrtoMic1 (Top) & SprktoMic2 (Bot) \\
\hline \hline $1^{\text {st }}$ Structural & 92.7 & $90-95$ & 3.04 & 3.18 \\
$1^{\text {st }}$ Acoustic & 126.1 & $120-130$ & 1.49 & 1.59 \\
$2^{\text {nd }}$ Acoustic & 165.0 & $163-173$ & -0.50 & -0.39 \\
$2^{\text {nd }}$ Structural & 262.6 & $260-270$ & 12.11 & 11.98 \\
\hline \multicolumn{2}{l||}{ Broadband $(40-1000 \mathrm{~Hz})$} & 0.46 & 0.48 \\
\hline
\end{tabular}

Table 5.7: Power Diode Controller (Both On) Performance Summary

\begin{tabular}{l|l|l||r|r}
\hline Mode & \multirow{2}{*}{$\begin{array}{l}\text { Frequency } \\
\text { (Hz) }\end{array}$} & $\begin{array}{l}\text { Reduction } \\
\text { Range }(\mathrm{Hz})\end{array}$ & \multicolumn{2}{|c}{ Reduction (dB) } \\
\cline { 4 - 5 } & & SpkrtoMic1 (Top) & SprktoMic2 (Bot) \\
\hline \hline $1^{\text {st }}$ Structural & 92.7 & $90-95$ & 6.99 & 0.20 \\
$1^{\text {st }}$ Acoustic & 126.1 & $120-130$ & 1.92 & 1.96 \\
$2^{\text {nd }}$ Acoustic & 165.0 & $163-173$ & -0.01 & 0.20 \\
$2^{\text {nd }}$ Structural & 262.6 & $260-270$ & 5.37 & 9.99 \\
\hline \multicolumn{2}{l|}{ Broadband $(40-1000 \mathrm{~Hz})$} & 1.06 & 0.70 \\
\hline
\end{tabular}



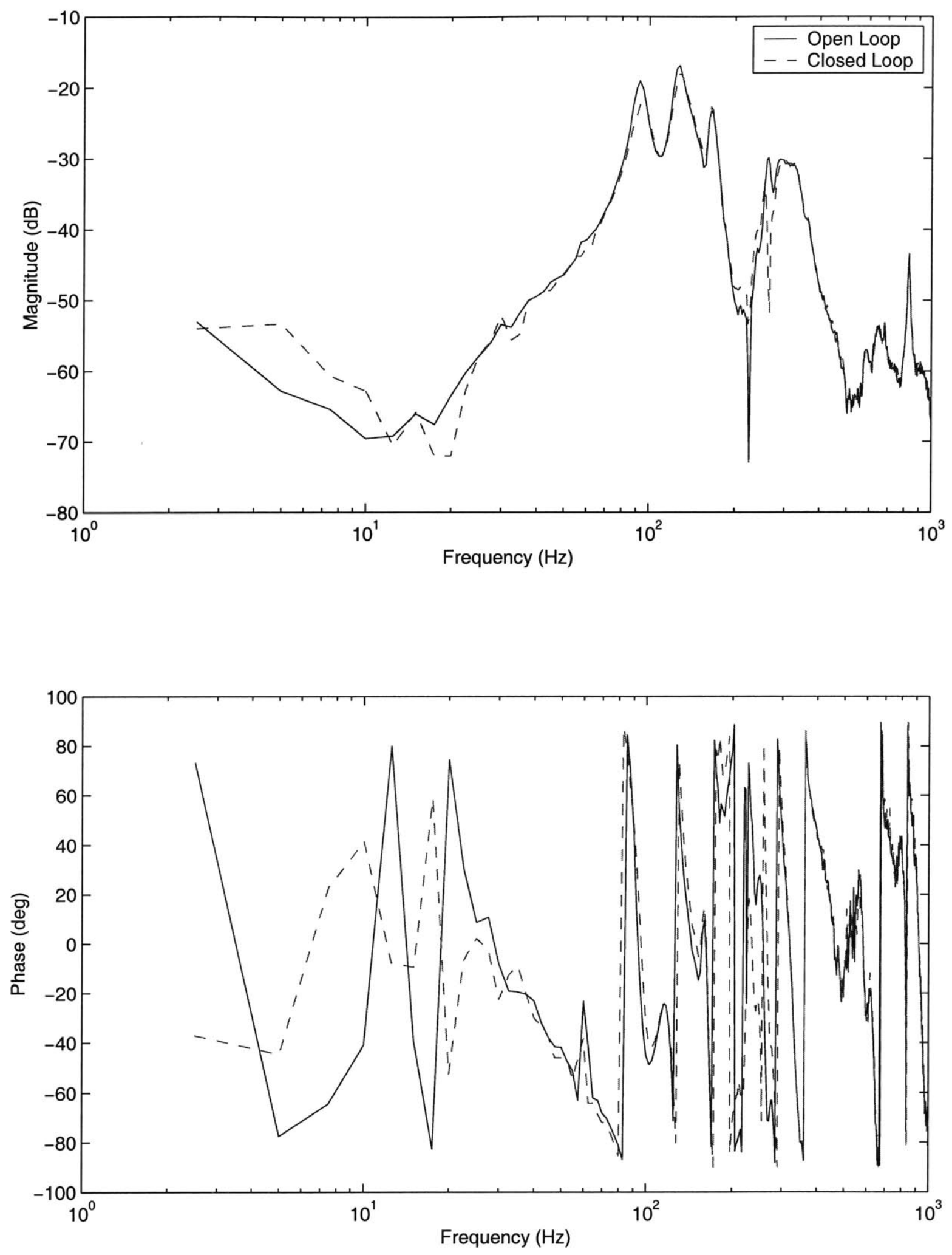

Figure 5-32: Power Diode Controller Performance (Bottom Plate System On Only) 

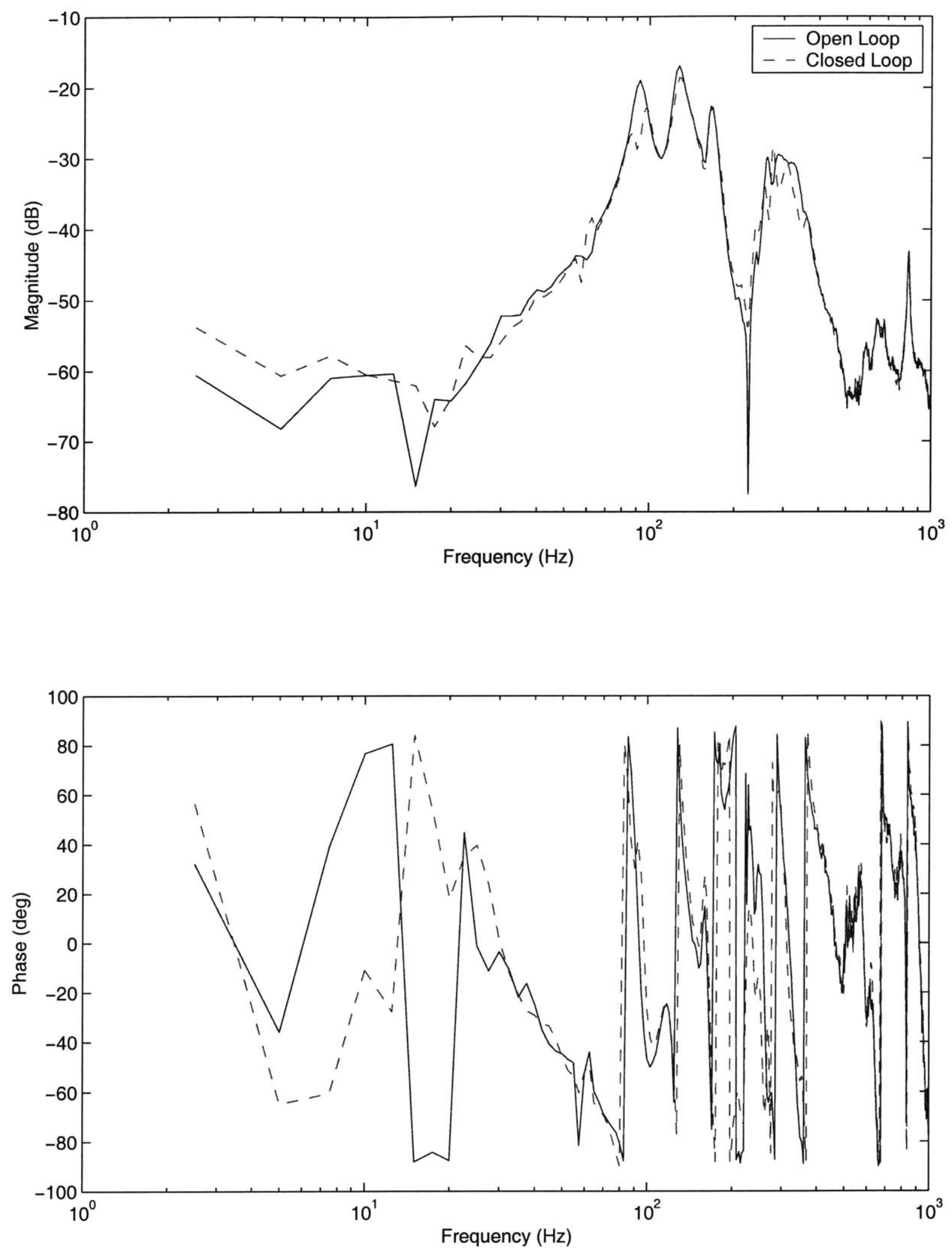

Figure 5-33: Power Diode Controller Performance (Both On) 

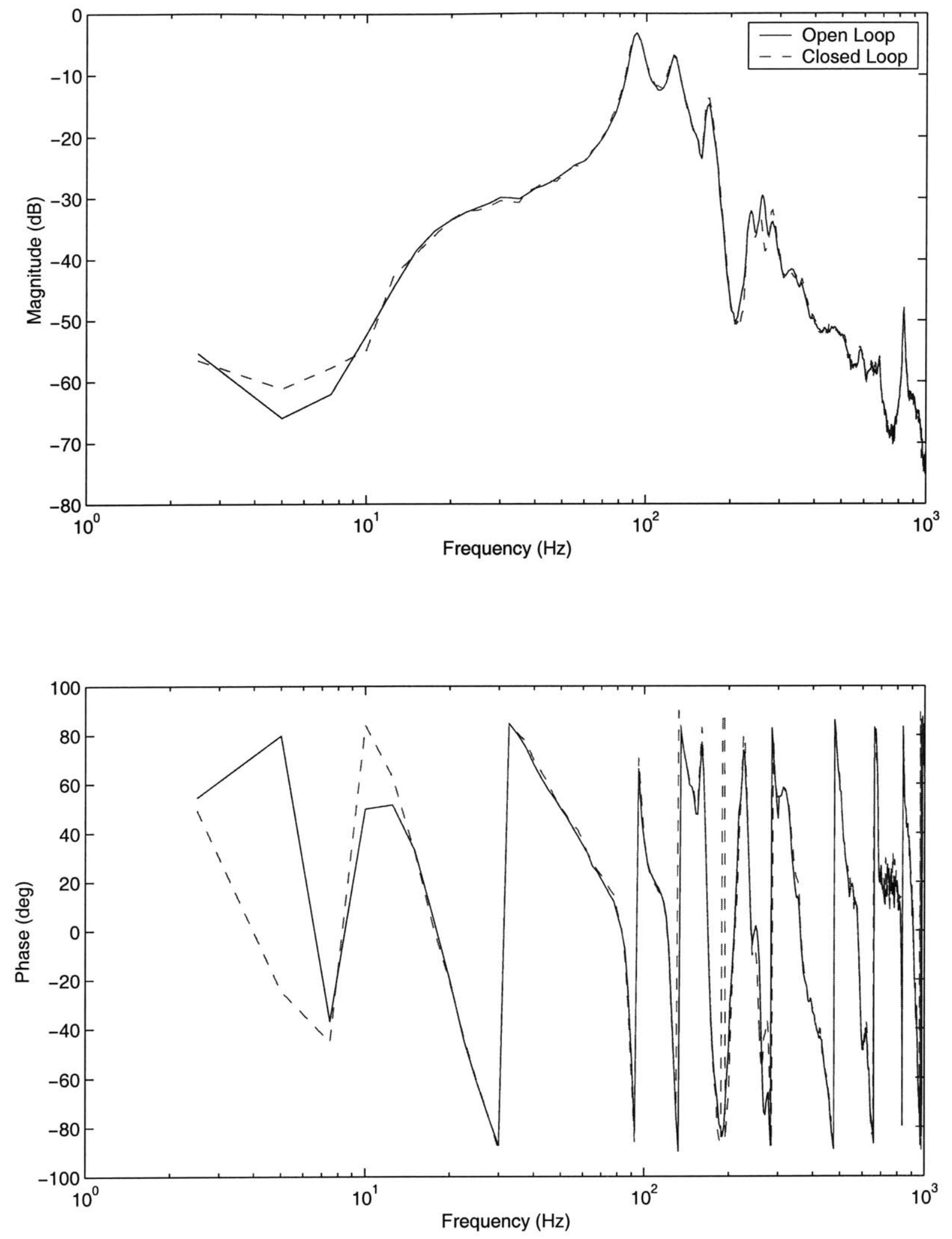

Figure 5-34: Power Diode Controller Reverse Direction (Both On) 


\subsubsection{Single Layer Reference}

Figure 5-35 shows the open and closed loop transfer functions using only a single layer for transmission control. The first structural mode at $81.7 \mathrm{~Hz}$ was reduced by $4.8 \mathrm{~dB}$ and the first acoustic mode near $160 \mathrm{~Hz}$ was reduced by $2.8 \mathrm{~dB}$. The broadband reduction from 40 to $1000 \mathrm{~Hz}$ was $1.80 \mathrm{~dB}$, which was better than the MIMO Controller and the Interference Controller but was slightly worse than the Successive Loop Closure Controller.

\subsubsection{Inter-plate Spacing}

The Successive Loop Closure Controller was designed not only for the inter-plate spacing of $9.875 \mathrm{in}$, but also for inter-plate spacings of 0.456 in and 1.163 in. The motivation for investigating the three spacings was to determine how close the plates could be placed without adversely affecting the controller effectiveness. For an interplate spacing of $0.456 \mathrm{in}$, the best broadband performance was a reduction of $0.91 \mathrm{~dB}$ over the range of $40-1000 \mathrm{~Hz}$. For the inter-place spacings of 1.163 in and 9.875 in, the broadband reductions for the same frequency spectrum were $2.0 \mathrm{~dB}$ and $2.1 \mathrm{~dB}$ respectively. This result suggests that the inter-plate spacing need not be large to obtain near-optimal performance, and that the plates can be spaced as close as about 1.2 in apart. 

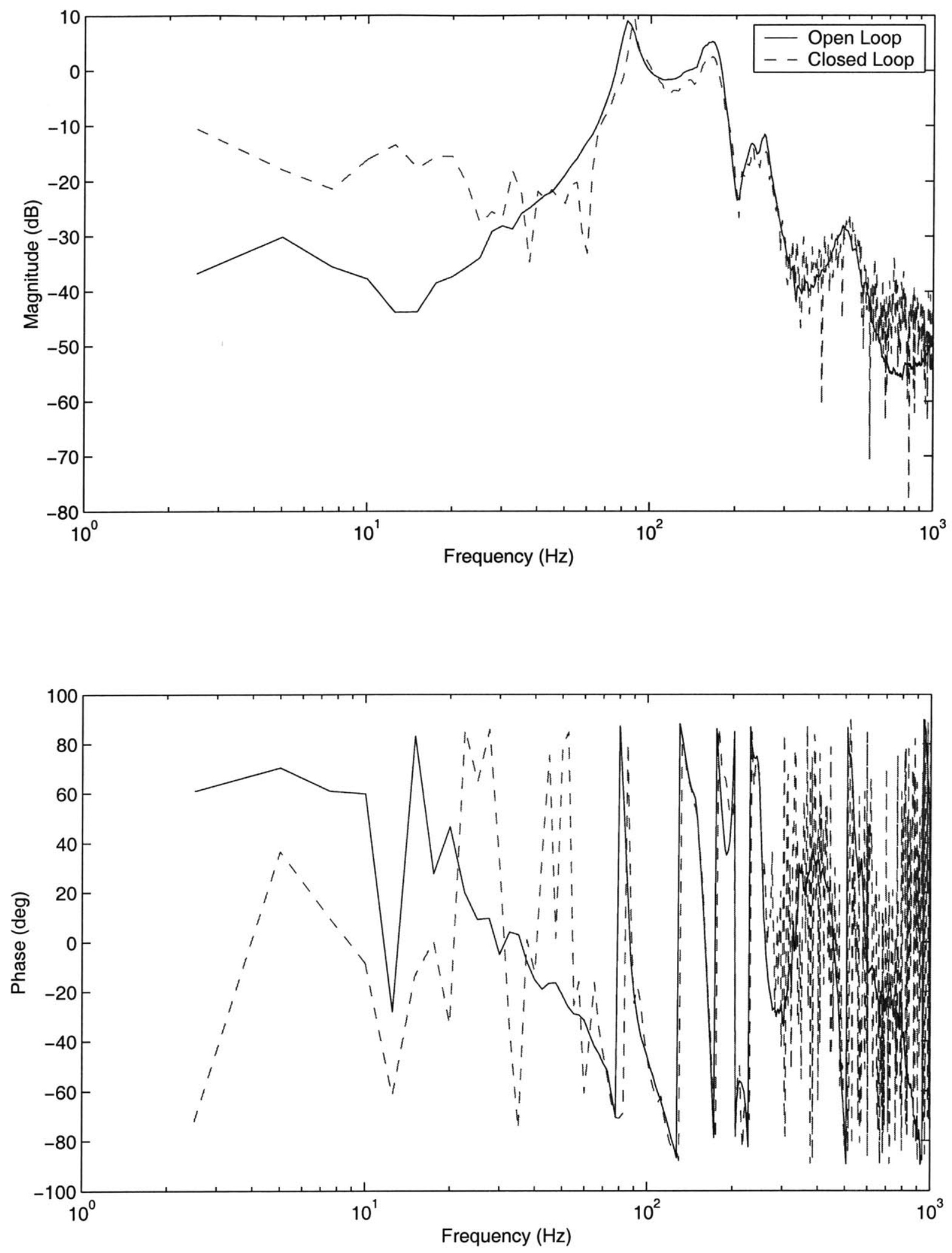

Figure 5-35: Single Layer Active Transmission Control 


\section{Chapter 6}

\section{Conclusions}

\subsection{Summary}

Active structural acoustic control (ASAC) has been proposed as a solution to the vibro-acoustic problems associated with launch vehicle shrouds. Although ASAC solutions have not yet been implemented in real launch vehicle shrouds, past and ongoing research have continued in the hope of improving ASAC design methodologies. This thesis investigated the use of active dual layer structural systems and by compared their performance against their active single layer counterpart.

The test chamber used in this thesis' experiments was an axis-symmetric cylinder which collapsed to a one-dimensional acoustic problem. The acoustics of the test chamber were simpler than the acoustics of the launch vehicle shroud, but the chamber preserved the relevant physical relationships of the problem while reducing the complexity and size of the large order multi-dimensional setup.

Past research done in the area [11] has suggested that ASAC is effective in reducing the amount of energy transmitted through the system by increasing the structural damping of the system. However, ASAC has been relatively ineffective at reducing the acoustic reverberation within the chamber because the structural-acoustic coupling is weak. Other solutions may prove to be more effective in addressing the acoustic reflection and reverberation problem such as using a plasma to control the acoustic field directly [19]. The results presented in this thesis verify the ineffectiveness of 
Table 6.1: Test Configuration Performance Summary

\begin{tabular}{l||r}
\hline Test Configuration & ${\text { Reduction }(\mathrm{dB})^{\dagger}}^{\dagger}$ \\
\hline \hline Single Layer (Controller On) & 1.80 \\
Single Thick Plate & 0.86 \\
Two Thin Plates & 1.80 \\
Large Single Controller $^{\ddagger}$ & 1.33 \\
Successive Loop Closure Controller $^{\ddagger}$ & 2.08 \\
Interference Controller $^{\ddagger}$ & 1.06 \\
Power Diode Controller $^{\ddagger}$ & 1.06 \\
\hline
\end{tabular}

ASAC in addressing the acoustic reflection problem. The Successive Loop Closure Controller and the Interference Controller support this claim as higher gains drove the system unstable at the second acoustic mode and that none of the attempts to control and reduce the magnitude of that mode's response worked. The mode was not sufficiently observable nor sufficiently controllable to be stabilizable at higher gains, and the low gain margins from the Nichols Charts confirmed this.

However, the various multi-layer transmission controllers designed, implemented and tested were able to confirm that multi-layer ASAC is a viable alternative to increasing the thickness of the structural layer. Among the controllers that were implemented and tested, the SLC Controller performed the best. It was able to achieve a broadband reduction of $2.08 \mathrm{~dB}$ over the $40-1000 \mathrm{~Hz}$ broadband range, compared to the $1.33 \mathrm{~dB}, 1.06 \mathrm{~dB}$ and $1.06 \mathrm{~dB}$ that were achieved using the MIMO Controller, the Interference Controller and the Power Diode Controller, respectively. These reductions represent the performance of the closed loop system relative to the open loop system and do not account for the introduction of the second structural plate. To determine the effectiveness of these multi-layer controllers relative to the single layer configuration, the broadband reduction in $\mathrm{dB}$ can be added to the $1.80 \mathrm{~dB}$ reduction associated with adding a second plate. Therefore, the SLC Controller represented a $3.86 \mathrm{~dB}$ broadband reduction in acoustic transmission over the single layer system with no active control. The results from all the test configurations are shown in Table 6.1. 


\subsection{Recommendations for Further Work}

The major limiting factor of the multi-layer controllers' achievable performance is the inability for the transmission controllers in targeting and controlling acoustic modes that are unobservable and are not stabilizable. Work done in the past confirm that ASAC is unable to solve the reflection control problem well, which suggests that another scheme has to be devised to solve the reflection control problem separately. In the past, control speakers have been used to address the reflection control problem, but their size and mass make them a poor solution to the payload fairing vibroacoustic problem where speaker placement locations are limited and mass constraints are tight.

A promising solution to the reflection control problem has been presented by Christopher Merchant [19]. He has proposed the use of a glow discharge plasma to address the reflection control problem and has achieved good broadband results (about $4 \mathrm{~dB}$ ). However, it remains to be seen how well the plasma actuator would work in an integrated multi-layer reflection and transmission control configuration. An analysis on the subject would answer the question and is the logical next step in the quest to solve the structural-acoustic problem.

\footnotetext{
${ }^{\dagger}$ Defined over the range $40-1000 \mathrm{~Hz}$

$\ddagger$ Reduction is relative to the configuration Two Thin Plates. If one wants the reduction relative to a single thin plate with no control, one would add $1.80 \mathrm{~dB}$ (value of Two Thin Plates relative to single thin plate with no control) to the value listed in the table.
} 


\section{Bibliography}

[1] Roger M. Glaese. Impedance Matching for Structural-Acoustic Control. PhD thesis, Massachusetts Institute of Technology, 1997.

[2] K. Eldred. Acoustic loads generated by the propulsion system. Technical Report SP-8072, NASA, 1971.

[3] Donald J. Leo and Eric H. Anderson. Vibroacoustic modeling of a launch vehicle payload fairing for active acoustic control. AIAA, 98-2086, 1998.

[4] P. Nelson, A. Curtis, S. Elliot, and A. Bullmore. The active minimization of harmonic enclosed sound fields, part I: Theory. Journal of Sound and Vibration, 117(1):1-13, 1987.

[5] P. Nelson, A. Curtis, S. Elliot, and A. Bullmore. The active minimization of harmonic enclosed sound fields, part II: A computer simulation. Journal of Sound and Vibration, 117(1):15-33, 1987.

[6] S. J. Elliot, P. A. Nelson, I. Slothers, and C. C. Boucher. In-flight experiments on the active control of propeller-induced cabin noise. Journal of Sound and Vibration, 140(2):219-238, 1990.

[7] C. R. Fuller and J. D. Jones. Experiments on reduction of propeller induced interior noise by active control of cylinder vibration. Journal of Sound and Vibration, 112(2):389-395, 1987.

[8] C. R. Fuller, S. D. Snyder, and C. H. Hausen. Active control of interior noise in model aircraft fuselage using piezo-ceramic actuators. AIAA, 90-3922, 1990. 
[9] Y. A. Lee. Study of helium effect on spacecraft random vibration with VAPEPS program. In Shock and Vibration Symposium, 59th, pages 119-135, Albuquerque, NM, October 1998.

[10] K. Weissman, M. E. McNelis, and W. D. Pordan. Implementation of acoustic blankets in energy analysis methods with application to the Atlas payload fairing. Journal of the Institute of Environmental Sciences (IES), pages 32-39, July/August 1994.

[11] Koji Asari. Vibroacoustic modeling and control for launch vehicle shrouds. Master's thesis, Massachusetts Institute of Technology, 1998.

[12] Carlos Guitérrez. Unidirectional active acoustic control for launch vehicle fairings. Master's thesis, Massachusetts Institute of Technology, 2000.

[13] Robert Pascal. Actuator and sensor design and modeling for structural acoustic control. Master's thesis, Massachusetts Institute of Technology, 1999.

[14] R. N. Jacques and D. W. Miller. Multivariable model identification from frequency domain data. IEEE Conference on Decision and Control, 1994.

[15] K. Liu. Identification of multi-input and multi-ouput systems by observability range space extraction. In IEEE Conference on Decision and Control, 31st, Tucson, AZ, December 1992.

[16] J. Moré. Lecture Notes in Mathematics 630: Numerical Analysis. SpringerVerlag, 1977.

[17] Kemin Zhou and John Doyle. Essentials of Robust Control. Prentice Hall, 1998.

[18] Robert G. Brown and Patrick Y. C. Hwang. Introduction to Random Signals and Applied Kalman Filtering. John Wiley \& Sons, 1997.

[19] Christopher Merchant. Active noise control using glow discharge plasma panels. Master's thesis, Massachusetts Institute of Technology, 2001.

$$
2766-30
$$

